\title{
NRC Multiphysics Analysis Capability Deployment FY 2021 - Part 1
}

\section{December 2020}

Sebastian Schunert ${ }^{1}$, Guillaume L. Giudicelli ${ }^{2}$, Paolo Balestra ${ }^{1}$, Javier Ortensi $^{1}$, Ramiro Freile ${ }^{3}$, and Logan Harbour ${ }^{3}$

${ }^{1}$ Reactor Physics Methods and Analysis

${ }^{2}$ Computational Frameworks

${ }^{3}$ Texas A\&M University

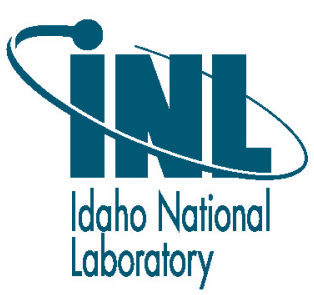




\section{DISCLAIMER}

This information was prepared as an account of work sponsored by an agency of the U.S. Government. Neither the U.S. Government nor any agency thereof, nor any of their employees, makes any warranty, expressed or implied, or assumes any legal liability or responsibility for the accuracy, completeness, or usefulness, of any information, apparatus, product, or process disclosed, or represents that its use would not infringe privately owned rights. References herein to any specific commercial product, process, or service by trade name, trade mark, manufacturer, or otherwise, does not necessarily constitute or imply its endorsement, recommendation, or favoring by the U.S. Government or any agency thereof. The views and opinions of authors expressed herein do not necessarily state or reflect those of the U.S. Nuclear Regulatory Commission. 
INL/EXT-20-60777

\title{
NRC Multiphysics Analysis Capability Deployment FY 2021 - Part 1
}

\author{
Sebastian Schunert ${ }^{1}$, Guillaume L. Giudicelli ${ }^{2}$, Paolo Balestra ${ }^{1}$, Javier Ortensi ${ }^{1}$, \\ Ramiro Freile ${ }^{3}$, and Logan Harbour ${ }^{3}$ \\ ${ }^{1}$ Reactor Physics Methods and Analysis \\ ${ }^{2}$ Computational Frameworks \\ ${ }^{3}$ Texas A\&M University
}

December 2020

Idaho National Laboratory

Reactor Physics Methods and Analysis

Idaho Falls, Idaho 83415

http://www.inl.gov

Prepared for the

Office of Nuclear Regulatory Research

U. S. Nuclear Regulatory Commission

Washington, D. C. 20555

Task Order No.: 31310019F0015 
Page intentionally left blank 


\section{SUMMARY}

This report details the progress and activities of Idaho National Laboratory (INL) on the Nuclear Regulatory Commission (NRC) project “Development and Modeling Support for Advanced NonLight Water Reactors."

The tasks completed for this report are:

- Task 1d: The net radiation transfer method was implemented into MOOSE for modeling Reactor Cavity Cooling System (RCCS) geometries. RCCS models for two experiments(1) Natural Convection Shutdown Heat Removal Test Facility (NSTF) experiment R022 and (2) High Temperature Test Reactor (HTTR) Vessel Cooling System (VCS) mockup-were created. For validation, computed temperature distributions were compared to measured temperatures.

- Task 4c: An algorithm for computing the pebble-bed reactor equilibrium core isotopic composition was developed, and an initial version was implemented into the reactor multiphysics code Griffin. Initial results for a simplified axisymmetric pebble-bed reactor are presented.

- Task 7: Generation of a reference plant model for molten-salt-cooled pebble-bed reactors. A coupled steady-state multiphysics model of the plant was constructed in the MOOSE framework using Griffin and Pronghorn. 
Page intentionally left blank 


\section{Contents}

Summary i i

\begin{tabular}{llr}
\hline 1 INTRODUCTION & 1
\end{tabular}

2 RCCS MODEL VALIDATION

2.1 Development of the Net Radiation Transfer Method in MOOSE . . . . . . . . . . . . 4

2.2 Natural Convection Shutdown Heat Removal Test Facility (NSTF) $\ldots . . . . . . . .8$

2.2 .1 Preliminary Verification $\ldots \ldots \ldots \ldots \ldots \ldots$

2.2.2 Comparison with Experimental Results . . . . . . . . . . . . . . . . . . . . . 14

2.3 High-Temperature Test Reactor Vessel-Cooling System . . . . . . . . . . . . . . . 17

3 PEBBLE-BED REACTOR EQUILIBRIUM CORE $\quad 24$

3.1 Outline of the Equilibrium Core Depletion Algorithm . . . . . . . . . . . . . . . 24

3.2 Governing Equations $\ldots \ldots \ldots \ldots \ldots \ldots \ldots \ldots$

3.2 .1 Streamlines . . . . . . . . . . . . . . . . . . . . . 27

3.2.2 $\quad$ Balance Equation for Pebbles on Streamlines . . . . . . . . . . . . . . . . 27

$3.2 .3 \quad$ Measuring Pebble Burnup . . . . . . . . . . . . . . . . . . . . 28

$3.2 .4 \quad$ Balance Equation for Pebble Types on Streamlines . . . . . . . . . . . . . . . . . . 30

$3.2 .5 \quad$ Balance Equation for Isotopes on Streamlines . . . . . . . . . . . . . . . . . . . . . 32

$3.2 .6 \quad$ Balance Equation for Pebble Types in the PHSS . . . . . . . . . . . . . . . . . 33

$3.2 .7 \quad$ Balance Equation for Isotopes in PHSS . . . . . . . . . . . . . . . . . . . . 36

3.3 Solution Algorithm and Data Transfers $\ldots \ldots$. . . . . . . . . . . . . . 37

3.4 Numerical Results . . . . . . . . . . . . . . . . . . . . . . . . . . . . . . . 39

\begin{tabular}{|lll}
4 & MOLTEN SALT PEBBLE-BED REFERENCE PLANT & 47
\end{tabular}

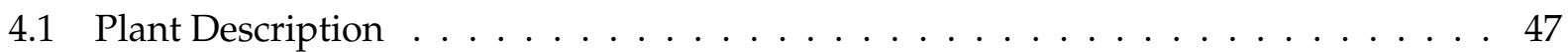

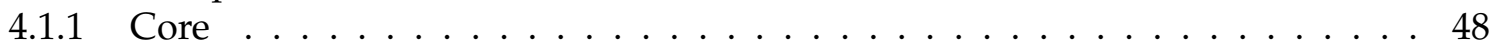

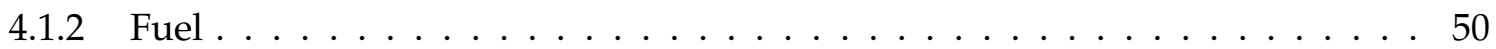

4.2 Neutronics Model . . . . . . . . . . . . . . . . . . . . . . . . . 50

$4.2 .1 \quad$ Cross-Section Generation $\ldots \ldots \ldots \ldots$

4.2 .2 Solution Method $\ldots \ldots \ldots \ldots \ldots \ldots$

4.2 .3 Cross-code validation . . . . . . . . . . . . . . . . . . 54

4.3 Thermal-Hydraulics Model $\ldots \ldots \ldots \ldots$. . . . . . . . . . . . . 56

$4.3 .1 \quad$ Model Description . . . . . . . . . . . . . . . . . . . . . 56

4.3 .2 Verification \& Validation $\ldots \ldots \ldots \ldots \ldots \ldots \ldots$

4.4 Fuel Performance Model $\ldots \ldots \ldots$. . . . . . . . . . . . . . . . . . 60

$4.4 .1 \quad$ Model Description . . . . . . . . . . . . . . . . . . . . . 60

4.4 .2 Avenues for Future Work $\ldots \ldots \ldots$. . . . . . . . . . . . . . 60

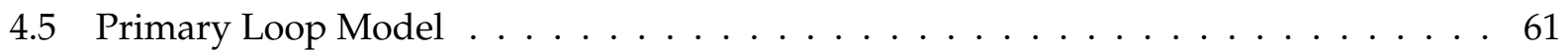

4.6 Multiphysics Coupling . . . . . . . . . . . . . . . . . . . . . 61

4.6 .1 Steady-State Simulations . . . . . . . . . . . . . . . . . . . . 61

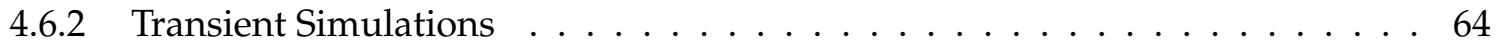

4.7 Future Work . . . . . . . . . . . . . . . . . . . . . . . . . . 64

\begin{tabular}{|lrl}
5 & CONCLUSIONS & 65
\end{tabular}

\begin{tabular}{ll}
\hline References & 66
\end{tabular} 
Page intentionally left blank 


\title{
ACRONYMS
}

\author{
INL Idaho National Laboratory \\ NRC Nuclear Regulatory Commission \\ NSTF Natural Convection Shutdown Heat Re- \\ moval Test Facility \\ RCCS Reactor Cavity Cooling System \\ SOW Statement-of-Work
}


Page intentionally left blank 


\section{INTRODUCTION}

This report details the progress and activities of Idaho National Laboratory (INL) in regard to the Nuclear Regulatory Commission (NRC) project “Development and Modeling Support for Advanced Non-Light Water Reactors."

Table 1 provides a summary of the tasks completed for this report. It matches the deliverable number, statement-of-work task, and (short) description of the deliverable with the relevant section in this report.

Table 1: List of deliverables.

\begin{tabular}{|c|c|c|c|}
\hline $\begin{array}{l}\text { Deliverable } \\
\text { Number }\end{array}$ & $\begin{array}{l}\text { SOW } \\
\text { Task }\end{array}$ & $\begin{array}{l}\text { Report } \\
\text { Section }\end{array}$ & Description \\
\hline $1 b$ & $1 d$ & 2 & $\begin{array}{l}\text { Perform RCCS model validation using data from the ANL flat panel } \\
\text { tests and HTTR-VCS tests }\end{array}$ \\
\hline $4 c$ & $4 c$ & 3 & $\begin{array}{l}\text { Report describing Mammoth-Rattlesnake example problem for pebble- } \\
\text { bed equilibrium burnup distribution and associated input model }\end{array}$ \\
\hline 7 & 7 & 4 & $\begin{array}{l}\text { Report describing reference plant model for molten-salt-cooled pebble- } \\
\text { bed reactor and associated input model }\end{array}$ \\
\hline
\end{tabular}

The tasks completed for this report are:

- Task 1d: The net radiation transfer method was implemented into MOOSE for modeling reactor cavity cooling system (RCCS) geometries. RCCS models for two experiments-(1) Natural Convection Shutdown Heat Removal Test Facility (NSTF) experiment R022 and (2) High Temperature Test Reactor (HTTR) Vessel Cooling System (VCS) mockup experiment I- were created. For validation, computed temperature distributions were compared to measured temperatures.

- Task 4c: An algorithm for computing the pebble-bed reactor equilibrium core isotopic composition was developed, and an initial version was implemented into the reactor multiphysics code Griffin. Initial results for a simplified axisymmetric pebble-bed reactor are presented.

- Task 7: A coupled steady-state neutronics, thermal-hydraulics, 1-D fuel performance model of the Mark-1 Pebble-Bed Fluoride-Salt-Cooled High-Temperature Reactor was created by 
coupling Griffin and Pronghorn. Model developments support future studies of operationally relevant transients and accident scenarios. 


\section{RCCS MODEL VALIDATION}

Passive decay heat removal during loss-of-flow accidents is important for high-temperature gascooled reactors (HTGRs) in terms of protecting the fuel and structural components from damage. A decay heat removal concept used in many HTGR designs is the reactor cavity cooling system (RCCS) [1-5]. While the design specifics of each RCCS may vary, the general concept is identical across all designs. The RCCS is a structure comprised of metal plates surrounding the reactor pressure vessel (RPV) at a distance of roughly 1-2 m. These plates are in thermal contact with standpipes or chimneys carrying either water or air via natural or forced circulation. Heat is transferred from the RPV to the RCCS by thermal radiation (80-90\%) and natural circulation within the cavity $(10-20 \%)$; the breakdown is affected by the reactor operating temperature and changes during relevant design-basis accidents (DBAs). For HTGRs, the RCCS usually has a height in excess of 10 $\mathrm{m}$ and features natural-circulation Rayleigh numbers of $\sim 1 \times 10^{13}$.

Modeling the RCCS under DBA conditions is important for ensuring (1) plant safety and (2) that the plant does not suffer unrecoverable damage (e.g., exceeding the temperature limit of the RPV). HTGR transients require modeling a significant fraction of the plant and can range over several days in duration. Therefore, detailed computational fluid dynamics (CFD) models are impractical, and the analysis must resort to system-level (using, for example, RELAP-5 [6], RELAP-7 [7], or SAM [8] codes) or intermediate fidelity (using, for example, Pronghorn [9] or AGREE [10]) analysis. However, these efficient, lower- or intermediate-fidelity models rely on modeling assumptions and correlations that must be validated in order to establish the accuracy of the analysis.

Section 2.1 describes the implementation of the net radiation transfer in MOOSE. We report the modeling results obtained with MOOSE for two experimental facilities: the Natural Convection Shutdown Heat Removal Test Facility (NSTF) [11] and the High Temperature Test Reactor (HTTR) Vessel Cooling System (VCS) mockup [12]. For the NSTF, we modeled the air-based transient R022; for the HTTR VCS, we modeled Experiment I with vacuum conditions. The models and corresponding results are presented in Sections 2.2 and 2.3 . 


\subsection{Development of the Net Radiation Transfer Method in MOOSE}

Modeling radiative heat transfer is important for the RCCS because $80-90 \%$ of the heat removed from the RPV is removed via radiation. For radiation heat transfer problems, the MOOSE heat conduction module [13] implements the net radiation transfer method (or "view factor method") as an approximation for solving the radiation transfer equation that is valid if (1) the medium in the cavity is transparent to the radiation and (2) the material properties do not depend on the energy of the radiation. The net radiation method simulates radiation as mono-energetic and takes absorption/re-emission into account using a single emissivity parameter.

In MOOSE, the net radiation transfer method implements the heat transfer within cavities. A cavity is a portion of the domain completely enclosed by sidesets. To improve resolution, the sidesets are divided into radiation patches, and the exchange of heat is computed between the radiation patches. In MOOSE, patches are grouped into three types: (1) ones that define a temperature variable on the other side of the sidesets away from the cavity, (2) ones with a constant temperature, and (3) adiabatic patches.

The net radiation transfer method is comprehensively described in Ref. [14]; hence, the equations are stated without derivation. We solve for $J_{j}$, defined as the radiosity of patch $j$. For adiabatic walls, we solve:

$$
\sum_{j=1}^{n}\left(\delta_{i, j}-F_{i, j}\right) J_{j}=0
$$

whereas for fixed temperature and variable temperature walls, we solve:

$$
\sum_{j=1}^{n}\left(\delta_{i, j}-\left(1-\epsilon_{i}\right) F_{i, j}\right) J_{j}=\beta_{i}
$$

In Eqs. 1 and $2, \delta_{i, j}$ is the Kronecker delta, $\epsilon_{i}$ is the emissivity of radiation patch $i, F_{i, j}$ is the view factor between patches $i$ and $j$ (to be defined more precisely later), $T_{i}$ is the temperature on radiation patch $i$, and $\beta_{i}$ is defined as:

$$
\beta_{i}=\frac{\epsilon_{i} \sigma}{A_{i}} \int_{A_{i}} T_{i}^{4}(\vec{r}) d A
$$

where $A_{i}$ is the area of radiation patch $i$, and $T_{i}$ is the temperature distribution on radiation patch $i$. 
The view factor, $F_{i, j}$, is defined as a double integral over patches $i$ and $j$. In 3-D geometries, the view factor, $F_{i, j}$, is computed by:

$$
F_{i, j}=\frac{1}{A_{i} \pi} \int_{A_{i}} \int_{A_{j}} \frac{\cos \alpha_{i} \cos \alpha_{j}}{r^{2}} d A_{i} d A_{j}
$$

where the integral is taken over points $\vec{r}_{i}$ on $A_{i}$ and $\vec{r}_{j}$ on $A_{j}$ on patches $i$ and $j$. For each combination of points $\vec{r}_{i}$ and $\vec{r}_{j}$, we define:

$$
\begin{aligned}
r & =\left\|\vec{r}_{j}-\vec{r}_{i}\right\| \\
\cos \alpha_{i} & =\frac{\vec{n}_{i} \cdot\left(\vec{r}_{j}-\vec{r}_{i}\right)}{r} \\
\cos \alpha_{j} & =\frac{\vec{n}_{j} \cdot\left(\vec{r}_{i}-\vec{r}_{j}\right)}{r}
\end{aligned}
$$

where $\vec{n}_{i}$ is the normal vector on patch $i$ pointing into the cavity and $\vec{n}_{j}$ is the corresponding counterpart on patch $j$. In 2-D geometries, the radiation patches become line segments $L_{i}$ and $L_{j}$, and the view factor is computed as:

$$
F_{i, j}=\frac{1}{2 L_{i}} \int_{L_{i}} \int_{L_{j}} \frac{\cos \alpha_{i, 0} \cos \alpha_{j, 0}}{r_{0}} d l_{i} d l_{j}
$$

where $\cos \alpha_{i, 0}, \cos \alpha_{j, 0}$, and $r_{0}$ are defined identically to $\cos \alpha_{i}, \cos \alpha_{j}$, and $r$ in the 3-D case, except that all distances and angles are measured in the plane.

The exchange of radiation in the cavity can be coupled to the solution of the heat conduction on blocks adjacent to the cavity (i.e., they share a sideset with the cavity). For these blocks, the heat conduction equation is given by:

$$
\rho(t, \vec{x}) c(t, \vec{x}) \frac{\partial T}{\partial t}-\nabla k(t, \vec{x}) \nabla T=\dot{q} \text { for } \vec{x} \in V
$$

where $T$ is temperature, $t$ is time, $\vec{x}$ is the vector of spatial coordinates, $V$ is the domain where heat conduction is solved, $\rho$ is the density, $c$ is the specific heat capacity, $k$ is the thermal conductivity, and $\dot{q}$ is a heat source. Radiative exchange in the cavity is coupled with the heat conduction 
equation via boundary conditions on radiation patches $A_{i}$, as given by:

$$
-k \vec{n} \cdot \nabla T-\sum_{j=1}^{I} F_{i, j}\left(J_{i}-J_{j}\right)=0 \text { for } \vec{x} \in A_{i}
$$

The view factors are computed using MOOSE's ray-tracing module [15]. We adopt the approach of subdividing each radiation patch into element faces, then applying a spatial quadrature rule on each element face. Next, we spawn rays from each quadrature point along the directions provided by an angular quadrature. View factors are computed by tracing the ray from its starting point to where it intersects another radiation patch; the view factor between the patch of origin and the destination patch is then incremented.

To facilitate the computation of view factors, we change the integration variable in Eq. 4 from $\vec{r}_{j}$ to angular direction $d \hat{\Omega}$. The infinitesimal element $d A_{j}$ becomes:

$$
d A_{j}=\frac{r^{2}}{\cos \alpha_{j}} d \hat{\Omega}
$$

where $d \hat{\Omega}=\sin \alpha_{i} d \alpha_{i} d \omega$ and $\omega$ is the azimuthal angle of $\hat{\Omega}$ measured in a plane orthogonal to $\vec{n}_{1}$ with respect to an arbitrarily chosen vector in that plane. The view factor is then computed by:

$$
F_{i, j}=\frac{1}{A_{i} \pi} \int_{A_{i}} \int_{\hat{\Omega} \in A_{j}} \cos \alpha_{1} d A_{i} d \hat{\Omega}=\frac{1}{A_{i} \pi} \int_{A_{i}} \int_{\hat{\Omega} \in A_{j}} \hat{\Omega} \cdot \vec{n}_{i} d A_{i} d \hat{\Omega}
$$

where $\hat{\Omega} \in A_{j}$ are all angular directions (starting from $\vec{r}_{i}$ ) that intersect $A_{j}$ before intersecting any other radiation patch. We define $\mu_{i}=\cos \alpha_{i}$ and finally obtain:

$$
F_{i, j}=\frac{1}{A_{i} \pi} \int_{A_{i}} \int_{\hat{\Omega} \in A_{j}} \mu_{i} d A_{i} d \hat{\Omega}
$$

It is noted that:

$$
\sum_{j=1}^{n} F_{i, j}=\frac{1}{A_{i} \pi} \int_{A_{i}} \int_{2 \pi^{+}} \mu_{i} d A_{i} d \hat{\Omega}=1
$$

The angular quadrature is selected to be a half-range Gauss-Legendre-Chebyshev quadrature adopted from Ref. [16] by first restricting the polar range to $0<\mu_{i}<1$ and rotating the angular directions so that the polar angle is measured with respect to $\vec{n}_{i}$ instead of $(0,0,1)$. When 
using spatial and angular quadratures, Eq. 12 becomes:

$$
F_{i, j}=\frac{1}{A_{i} \pi} \sum_{e \in A_{i}} \sum_{l \in e} \sum_{q \in A_{j}} w_{s, l} w_{a, q} \mu_{q, i}
$$

where $e$ are the elements in radiation patch $i, l$ indexes the quadrature points on element $e, q$ indexes the angular directions that lead to intersections with radiation patch $A_{j}, w_{s, l}$ is the l-th spatial weight, and $w_{a, q}$ is the q-th angular weight. In the MOOSE implementation, rays are started into the direction provided by the angular quadrature set without knowing a priori which radiation patch they ultimately intersect. Once an intersection with radiation patch $j$ is detected, the ray is killed, and the view factor, $F_{i, j}$, is incremented by $w_{s, l} w_{a, q} \mu_{q, i}$.

The net radiation transfer method implemented in MOOSE conserves energy if the view factors satisfy the following conditions:

$$
\begin{aligned}
\sum_{j=1} F_{i, j} & =1 \text { for all } i=1, \ldots, n \\
A_{i} F_{i, j} & =A_{j} F_{j, i} \text { for all } i<j \text { and } i=1, \ldots, n
\end{aligned}
$$

The first condition is always satisfied by the ray-tracing algorithm, but the second condition (the reciprocity condition) is only satisfied to within a discretization error. To ensure that energy is conserved in the radiation transfer computation, an additive correction of the view factor is performed. This additive correction ensures that the reciprocity condition is satisfied without inadvertently invalidating compliance with the first condition. To this end, we use the following additive correction:

$$
F_{i, j}^{\prime}=F_{i, j}+\Delta_{i, j}
$$

We compute the correction by solving the following constrained minimization problem:

$$
\begin{aligned}
\sum_{i, j=1}^{n} \Delta_{i, j}^{2} & \rightarrow \min \\
A_{i}\left(F_{i, j}+\Delta_{i, j}\right) & =A_{j}\left(F_{j, i}+\Delta_{j, i}\right) \text { for all } i<j \text { and } i=1, \ldots, n \\
\sum_{j=1}^{n} \Delta_{i, j} & =1-\sum_{j=1}^{n} F_{i, j} \text { for all } i=1, \ldots, n
\end{aligned}
$$


We use Lagrange multipliers to compute a linear system of equations for the unknown $\Delta_{i, j}$.

\subsection{Natural Convection Shutdown Heat Removal Test Facility (NSTF)}

In this subsection, we focus on experiment R022, performed for the air-cooled configuration of the NSTF; the specifications of the NSTF facility were adopted from Ref. [11], and the measurement results were digitized from Ref. [17]. In addition to a comparison with measured temperatures, we will present a comparison with the STAR-CCM+ model of NSTF experiment R022.

The geometry of the NSTF during the air-based experimental campaign is depicted in Fig. 1 . At the core of the facility is the heated cavity, which is $6.7-\mathrm{m}$ tall, 1.32-m wide, and 1.21-m deep. Twelve riser channels $(0.256-\mathrm{m}$ deep and $0.053-\mathrm{m}$ wide) run along the height of the cavity; the midpoint of the riser channels is located $0.834 \mathrm{~m}$ from the heated west wall. The pitch of the riser channels (in the N-S direction) is $0.1016 \mathrm{~m}$, and the midpoint distance of the left and rightmost channels to the north/south walls is also $0.1016 \mathrm{~m}$. The riser channels have walls of 0.005 m. The heated west wall and riser channels are made of low-carbon steels, the north and south walls are made from insulating material, while the east wall consists of a 1/8-in. plate of Al 3003 surrounded by insulating material. The top and bottom of the cavity are assumed to be made of insulating material (the authors could not confirm this from the final report [11]). The riser channels extend $0.41 \mathrm{~m}$ into the outlet plenum and $0.18 \mathrm{~m}$ into the inlet plenum. The properties of these materials are listed in Table 2, while the geometrical specifications of the cavity are summarized in Table 3

The NSTF facility is designed to facilitate natural-circulation cooling of the heated cavity. Heat is transferred to the riser channels via radiation and the natural convection heating of air inside the riser channels, enabling buoyancy to drive the flow through the system. The flow enters the facility from inside the building, moving into the inlet downcomer and plenum, and then into the riser ducts. From there, it reaches the outlet plenum, which connects the chimney stacks that discharge to the outside of the building that houses the NSTF.

This work focuses on experiment R022, which replaces natural convection with forced convection and matches the mass flow rate of a previous natural-circulation experiment. In modeling experiment R022, it is unnecessary to model the entire facility. We reduce the model to the inlet 
and outlet plena, the heated cavity, and the riser channels. For transient R022, the mass flow rate is fixed at $\dot{m}=0.605 \mathrm{~kg} / \mathrm{s}$, and the thermal power transferred into the cavity is estimated at 56 kW.
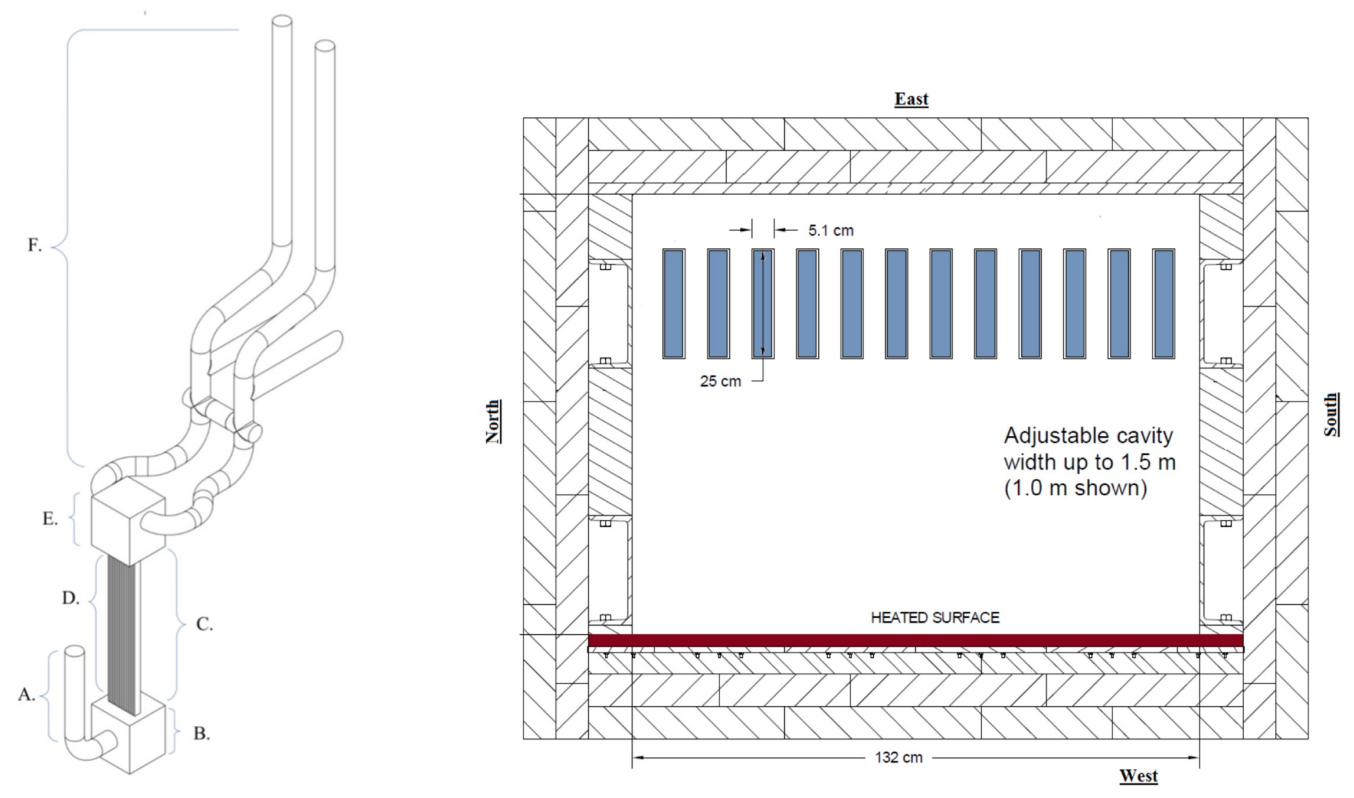

Figure 1: Left: Main segments of the NSTF facility: A. inlet downcomer, B. inlet plenum, C. heated cavity, D. riser ducts, E. outlet plenum, F. chimney stacks (picture courtesy of [18]). Right: Topdown view of the heated cavity (picture courtesy of [11]).

Table 2: Material properties of cavity materials.

\begin{tabular}{|c|c|c|c|}
\hline Material & Location & $\begin{array}{l}\text { Thermal } \\
\text { conductivity }[\mathrm{W} / \mathrm{m} \mathrm{K}]\end{array}$ & Emissivity [-] \\
\hline Low-carbon steel & Riser channel, west wall & 51.9 & 0.785 \\
\hline Insulator & $\begin{array}{l}\text { Bottom, top, north, south } \\
\text { walls; east wall following } \mathrm{Al}\end{array}$ & small & 0.2 \\
\hline Al 3003 & east wall paneling & not used ${ }^{a}$ & 0.4 \\
\hline
\end{tabular}

${ }^{a} \mathrm{Al} 3003$ is not modeled in the heat conduction problem, but an emissivity of 0.4 is adopted for the surface.

The MOOSE model consists of two components: (1) a 3-D heat-conduction problem coupled with radiation transfer in the cavity, and (2) a 0-D/1-D thermal-hydraulics model of the inlet plenum and riser channels developed in RELAP-7 [7].

The heat conduction model is depicted in Fig. 2; a cross-sectional view is provided on the left, and a 3-D view is provided on the right. We use symmetry to only model half the cavity. On the symmetry line, we apply a ReflectingRayBC that specularly reflects rays; the symmetry line 
Table 3: Specification of the NSTF cavity (all units are in $\mathrm{m}$ ).

\begin{tabular}{ll}
\hline Height & 6.7 \\
Width (N to S) & 1.32 \\
Depth (W to E) & 1.2065 \\
Riser width (N to S) & 0.053 \\
Riser depth (W to E) & 0.256 \\
West wall to riser midpoint (W to E) & 0.834 \\
Riser pitch & 0.1016 \\
Distance N/S to first riser midpoint (N to S) & 0.1016 \\
Thickness of riser channel walls & 0.005 \\
Extension of riser into inlet plenum & 0.18 \\
Extension of riser into outlet plenum & 0.41 \\
Thickness of heated steel plate & 0.0254 \\
Thickness of insulator (N, S, E) & 0.1016 \\
Thickness of insulator (bottom/top) & 0.0254 \\
\hline
\end{tabular}

sideset does not otherwise participate in the net radiation transfer calculation. The insulators on the north, south, and east walls have a thickness of $0.1016 \mathrm{~m}$, while the thickness of the top and bottom insulating walls is $0.0254 \mathrm{~m}$. The following simplifications and assumptions were adopted:

- The Al 3003 liner on the east wall is not modeled for the heat conduction solve and is replaced by insulator material. We do not expect this to change the results significantly. The Al 3003 and the insulator can be thought of as serial thermal resistors. The higher thermal conductivity of the Al 3003 does not significantly affect the very large thermal resistance; therefore, replacing the aluminium with the insulator does not affect the results. Note that we use an emissivity of 0.4 (corroded aluminium) on the east wall.

- We apply adiabatic boundary conditions on the outside of the insulator. We do not expect this to significantly change the results, since the high thermal resistance of the insulator prevents effective heat transfer across the insulated walls.

- The riser channels' corners are modeled as sharp corners, and the corner radius of $0.01 \mathrm{~m}$ is neglected.

- The outer surfaces of the risers that extend past the top and bottom of the cavities are modeled as adiabatic.

In addition to radiative heat transfer, heat transfer through natural convection is considered 
in the cavity. In lieu of a costly CFD solution, we use correlations that provide Nusselt numbers for the heat exchange of vertical and horizontal plates with a bulk temperature, $T_{\infty}$. The bulk temperature is computed from an energy balance of the air in the cavity:

$$
\sum_{i=1}^{n} h_{i} \int_{A_{i}}\left(T(\vec{r})-T_{\infty}\right) d A=0
$$

where $i$ indexes the sidesets bounding the cavity and $h_{i}$ is the heat transfer coefficient on surface $i$. We assume that, at steady-state, no mass flow of air enters or leaves the cavity. The heat transfer correlations used to compute $h_{i}$ are listed in Table 4. The Nusselt number, $\mathrm{Nu}$, is related to the heat transfer coefficient, as per:

$$
\mathrm{Nu}_{i}=\frac{h_{i} L_{i}}{k_{\text {air }}}
$$

where $L_{i}$ is the characteristic length of surface $i$ and $k_{\text {air }}$ is the thermal conductivity of air evaluated at temperature $\bar{T}_{i}=\left(T+T_{\infty}\right) / 2$. The correlations for natural convection are given in terms of the Rayleigh number, Ra, defined by:

$$
\mathrm{Ra}=\frac{g\left|T-T_{\infty}\right| L_{i}^{3}}{\bar{T}_{i} v^{2}} \operatorname{Pr}
$$

where $g=9.81 \mathrm{~m} / \mathrm{s}^{2}, v$ is the kinematic viscosity of air evaluated at $\bar{T}_{i}$, and Pr is the Prandtl number evaluated at $\bar{T}_{i}$.

Table 4: Natural convection heat transfer correlations by cavity surface. Rayleigh number Ra computed using Eq. 19 .

\begin{tabular}{llll}
\hline Type & Surfaces & $\begin{array}{l}\text { Characteristic } \\
\text { length } L_{i}\end{array}$ & Correlation \\
\hline Vertical Plate & $\begin{array}{l}\text { north, south, east, west } \\
\text { all cavity-facing riser surfaces }\end{array}$ & $\begin{array}{l}\text { Height } \\
A_{i} / P_{i}^{a}\end{array}$ & $0.1 \mathrm{Ra}^{1 / 3}[19]$ \\
Horizontal Plate & top, bottom & $0.15 \mathrm{Ra}^{1 / 3}[20]$ \\
\hline${ }^{a} P_{i}$ Perimeter. & &
\end{tabular}

Details of the 3-D MOOSE model (model name, element count, radiation patch count, and rays per quadrature point) are presented in Table 5. We perform one spatial refinement step, increasing the element count from $3 \times 10^{5}$ to $2.8 \times 10^{6}$, and one angular refinement step, increasing the number of rays from 512 per quadrature point to 960 per quadrature point. The RELAP-7 model comprises a VolumeJunction1Phase, with one inlet channel carrying $0.3025 \mathrm{~kg} / \mathrm{s}$ (half the total mass flow 

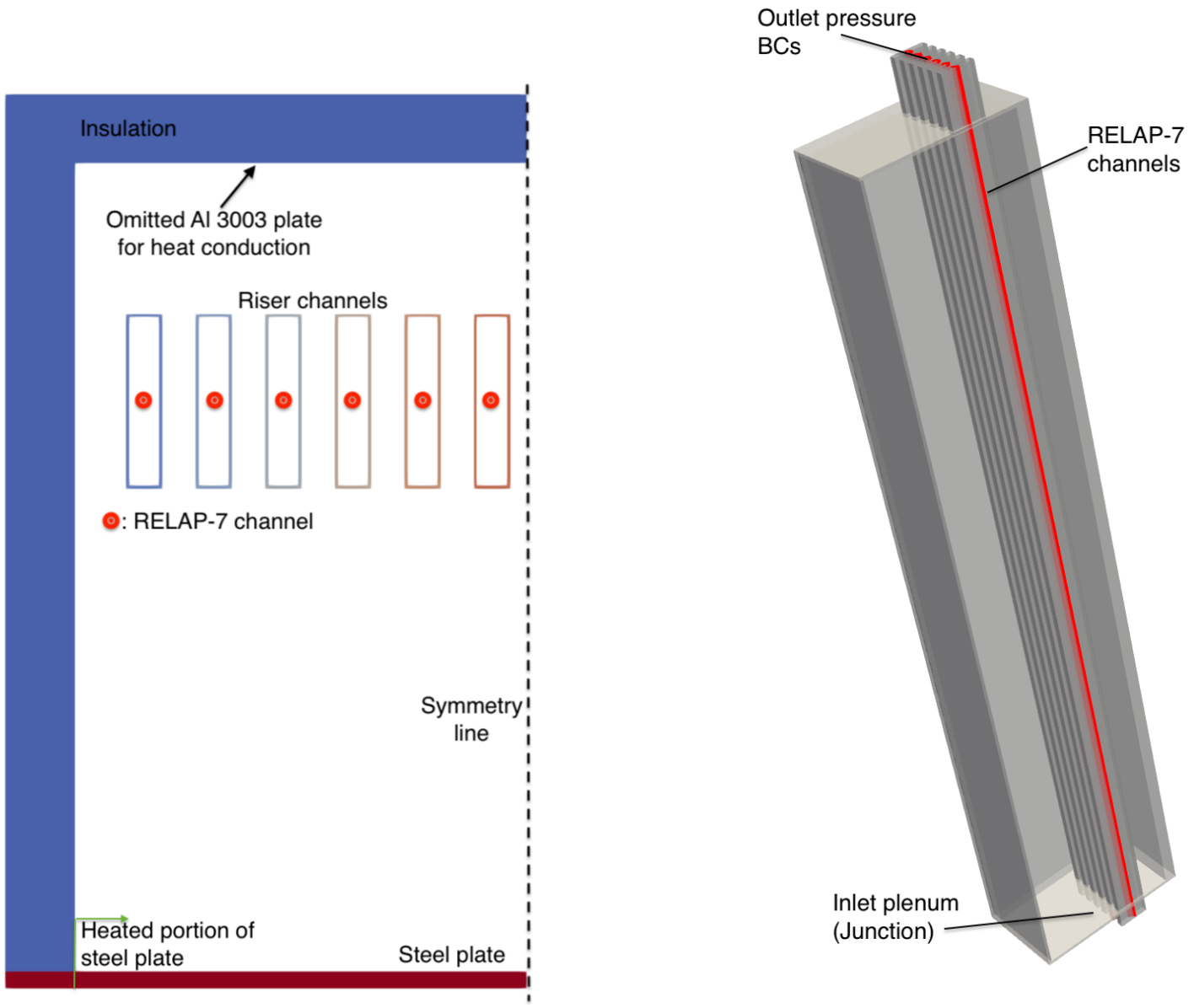

Figure 2: Left: Cross-sectional view of the MOOSE cavity model. Right: Cutaway view of the MOOSE model, including the RELAP-7 channels and the location of the inlet plenum.

rate) and six riser channels. The riser channels are discretized using 100 elements and RELAP-7's constant HLLC discretization (discontinuous Galerkin method with constant approximation). The 3-D heat conduction problem and RELAP-7 communicate by exchanging fluid temperatures, wall temperatures, and heat transfer coefficients averaged over the channel circumference but allowed to vary axially; the approach is detailed in Ref. [21]. The heat transfer coefficients are computed by RELAP-7 using Trace closures [22].

Four variants of the MOOSE model are considered, and these are referred to as $(a)-(d)$ :

(a) Heat conduction in the cavity does not include convection.

(b) Heat conduction in the cavity includes convection via heat transfer coefficients, $h_{i}$, along with a single cavity temperature, $T_{\infty}$. 
(c) Same as (b), but we modulate the temperature in the cavity via:

$$
T_{\infty}(z)=T_{\infty}+2 \Delta T \frac{z-z_{\operatorname{mid}}}{z_{\max }-z_{\min }}
$$

where $z_{\max }$ is the $z$-coordinate of the top of the cavity, $z_{\min }$ is the $z$-coordinate of the bottom of the cavity, $z_{\text {mid }}=\left(z_{\max }+z_{\min }\right) / 2$, and $\pm \Delta T$ is the maximum deviation of the local temperature from the average of $T_{\infty}$; we select $\Delta T=20 \mathrm{~K}$ and $\Delta T=40 \mathrm{~K}$, referred to as (c 20) and (c 40), respectively.

(d) No convection in the cavity or the riser channels. We apply a Dirichlet boundary at the inside of the riser channels, varying the temperature axially from 300-400 over the total length of the riser (i.e., including the top and bottom pieces extending into the inlet and outlet plena).

Table 5: Levels of model refinement for the MOOSE NSTF model.

\begin{tabular}{llll}
\hline Model name & Element count & Radiation patch count & Rays per quadrature points \\
r0 & 348,348 & $80 / 100 / 100^{a}$ & 512 \\
r1 & $2,786,784$ & $80 / 400 / 200$ & 512 \\
r1-a1 & $2,786,784$ & $160 / 800 / 400$ & 960 \\
\hline${ }^{a} P_{i}$ Riser sidesets/west wall/north, bottom, top.
\end{tabular}

The commercial code Star-CCM+ v14.04 is utilized to model experiment R022 in addition to the MOOSE model. The goal of this cross-comparison is to distinguish between potential coding mistakes in MOOSE and discrepancies between the MOOSE model and the as-built and as-performed experiment. The salient features of the STAR-CCM+ model are:

- The STAR-CCM+ model solves Navier-Stokes equations in the channels instead of the simpler and faster TH model used in MOOSE. To this end, STAR-CCM+ solves Reynoldsaveraged Navier-Stokes equations using the realizable $k-\epsilon$ model with Wolfstein's twolayer formulation [23]. The model uses a second-order finite volume discretization and the SIMPLE pressure-velocity coupling algorithm.

- The north, east, bottom, and top walls are modeled as adiabatic. The insulator behind these boundaries is not modeled. 
- Natural convection in the cavity is not modeled, so comparison can only be performed between the STAR-CCM+ model and MOOSE models (a) and (d).

- The curvature at the corner of the riser channels is explicitly modeled.

- The number of elements in the STAR-CCM+ model is 715, 236 in the heat conduction regions and 5,134,601 in the riser channel flow regions. The heated plate is discretized into 221,619 element faces, each of which is a radiation patch (the other walls in the cavity are discretized similarly). STAR-CCM+ uses 4,096 rays per radiation patch when computing view factors.

- In any other respect the MOOSE and STAR-CCM+ models are equivalent.

\subsubsection{Preliminary Verification}

Preliminary verification is performed for NSTF configuration (d). We compare lineouts of temperatures taken from the cavity-facing heated steel plate at $0.1525 \mathrm{~m}$ from the centerline $(0.508 \mathrm{~m}$ from the north wall and $0.6605 \mathrm{~m}$ from the leftmost wall of the MOOSE model) between MOOSE models $r 0$ and $r 1-a 1$ and the STAR-CCM+ model; the location of the lineout coincides with a column of thermocouples mounted in the experiment.

The lineouts are plotted in Fig. 3. MOOSE and STAR-CCM+ agree reasonably for the base r0 model, and one additional refinement step in MOOSE leads to excellent agreement. The slight disagreement in the corners is most likely caused by STAR-CCM+ modeling the walls as adiabatic sidesets while MOOSE explicitly models the insulator material. These results (and other simpler verification cases) provide evidence that MOOSE's net radiation transfer algorithm is implemented correctly.

\subsubsection{Comparison with Experimental Results}

This section compares various computational results of model variants (a)-(c) with experimental results. Neither Ref. [11] nor [17] provides tables of thermocouple IDs paired with measured temperatures. Therefore, the experimental results are retrieved from Ref. [17] (Fig. 5-28) using the digitization program WebPlotDigitizer. The referenced figure plots the thermocouple temperature vs. thermocouple ID. The digitization process is insufficient to resolve the exact ID of each ther- 


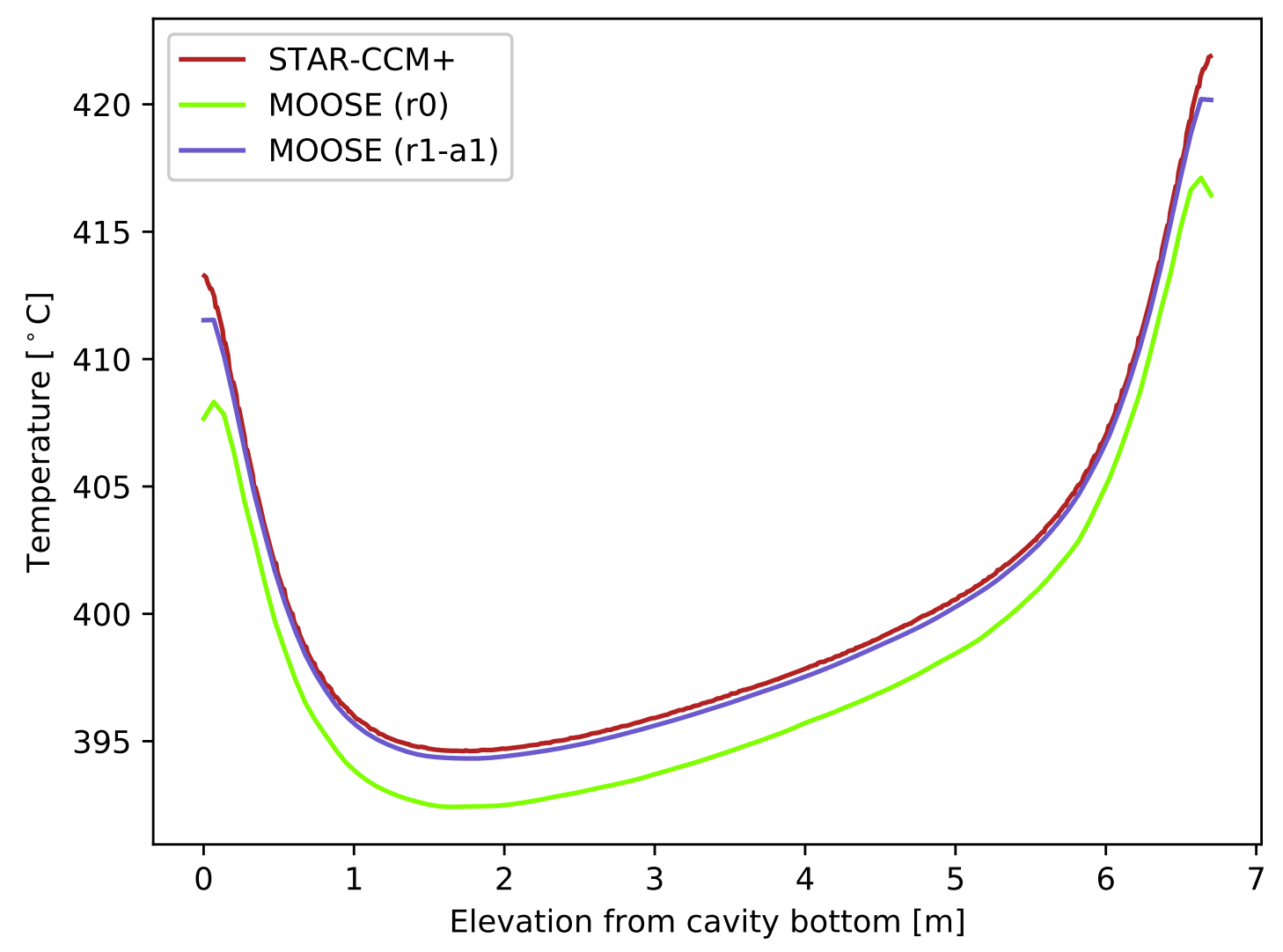

Figure 3: Lineout of the temperature along the z-direction of the heated cavity wall at a distance of $0.1525 \mathrm{~m}$ from the center.

mocouple. However, the thermocouple IDs roughly correlate with their elevation in the cavity because they are numbered in increasing order from bottom to top, north to south. We convert a thermocouple ID into elevation in the cavity via:

$$
z(i)=\frac{i}{i_{\max }} H
$$

where $H=6.7 \mathrm{~m}$ is the height of the cavity and $i_{\max }=126$ is the total number of thermocouples. This process washes out the position of the thermocouples in the lateral direction, leading to significant uncertainty when comparing experimental and computational results, since it is unclear which measured data should be compared to the computational results.

Lineouts of temperature $0.1525 \mathrm{~m}$ from the centerline are plotted in Fig. 4 , along with the 
experimental results. We observe the following:

- If no natural convection in the cavity is considered (Case [a]), the computational models overpredict the temperatures by about $40 \mathrm{~K}$. STAR-CCM+ and MOOSE agree well but show differences of a few degrees Kelvin at higher elevations, due to the different flow models in the riser channels (thermal-hydraulics vs. CFD).

- If natural convection in the cavity is considered (Case [b]), the MOOSE-predicted average temperature agrees well with the experimental results. However, the experimental results follow a steeper slope, meaning that MOOSE overpredicts temperatures at the bottom and underpredicts them at the top.

- If we impose a temperature distribution of $T_{\infty}$, the computational results' slope steepens because the heat transfer at the top is reduced and the heat transfer near the bottom is enhanced.

- Agreement between the model variant (d 40) and the experimental results falls within the uncertainty introduced by how the experimental results were extracted from Ref. [17]. Access to the raw data is necessary to confidently determine whether experimental and numerical results agree.

- Computational results show increased temperature near the top and bottom walls, while the experimental results do not. This increased temperature is caused by decreased radiative exchange with cool surfaces in the cavity and is therefore expected. It is unknown why the experimental results do not show a similar trend. 


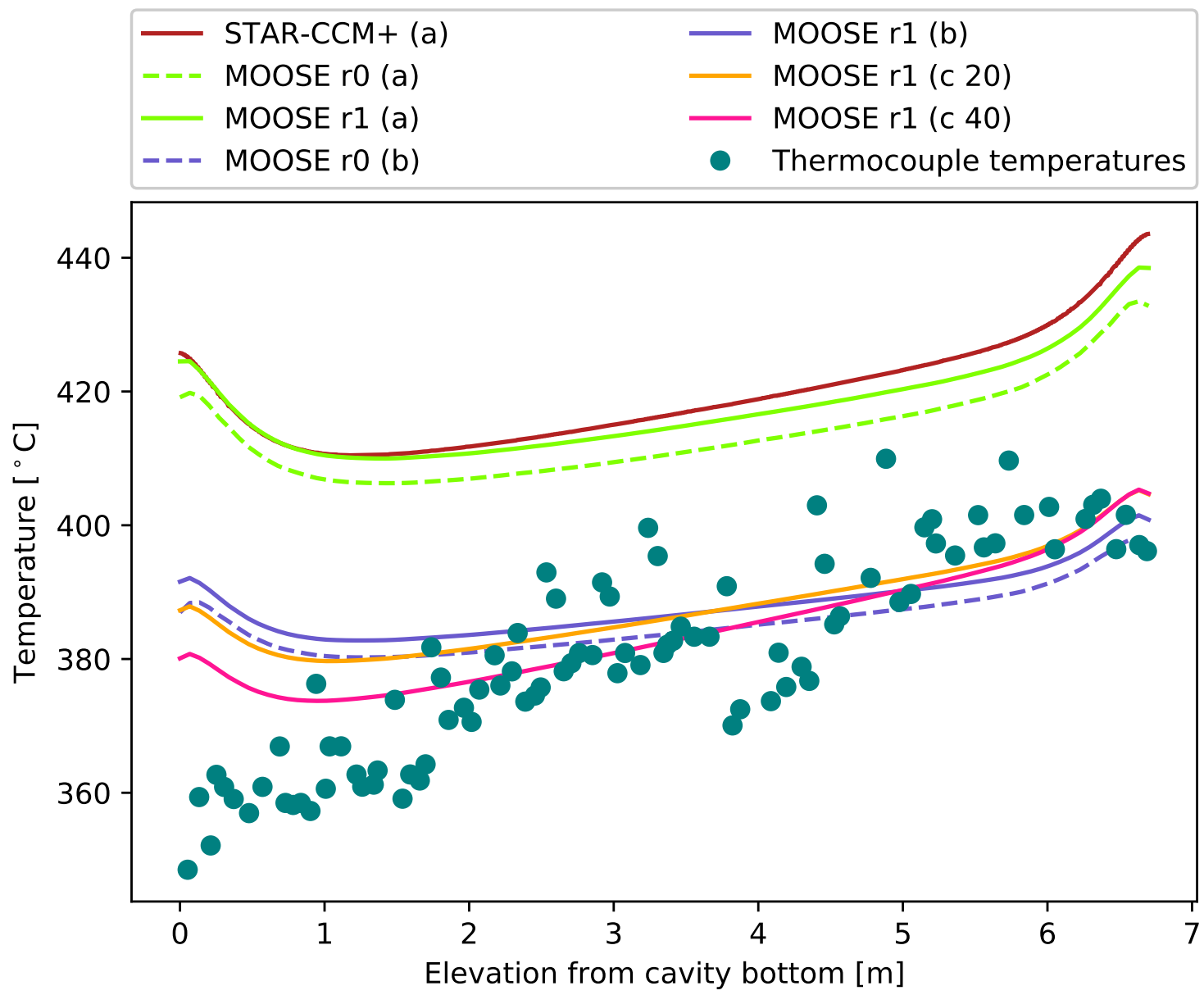

Figure 4: Computational results obtained from MOOSE and STAR-CCM+ compared to experimental results digitized from Ref. [17] and converted from thermocouple ID into elevation in the cavity. The computational results are lineouts from bottom to top on the heated surface of the cavity at a distance of $0.1525 \mathrm{~m}$ from the symmetry line.

\subsection{High-Temperature Test Reactor Vessel-Cooling System}

An RCCS mockup experiment was built in Japan to assess the effectiveness of the vessel-cooling system (VCS) for the HTTR [24]. The geometry of the VCS experiment is shown in Fig. 5. The scaled-down experimental apparatus consists of a heater located inside an RPV and a set of cooling panels located in the cavity walls below, above, and on the side of the RPV. The key dimensions are provided in Table 6. 


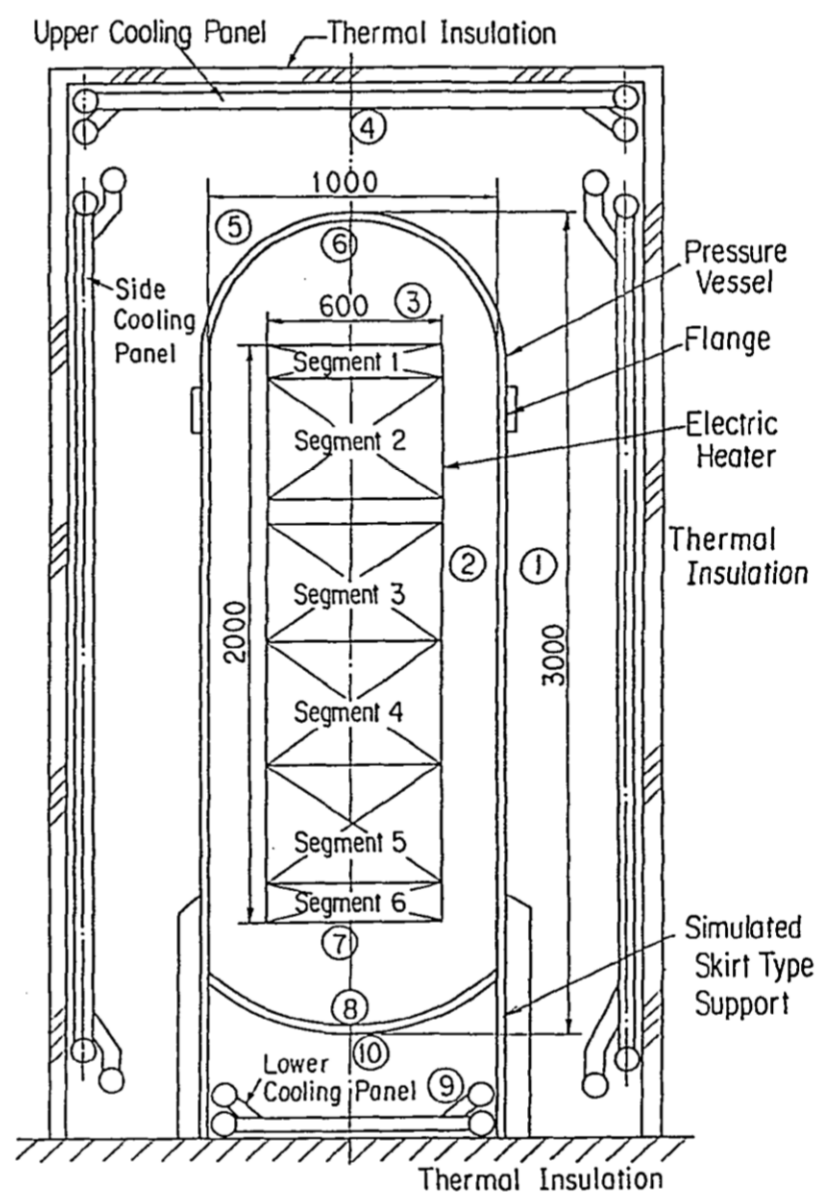

Figure 5: Layout of the VCS experimental apparatus.

Table 6: Specification of the VCS experiment (all units in $\mathrm{m}$ where not indicated).

\begin{tabular}{ll}
\hline Heater height & 2.0 \\
Heater inner radius & 0.2075 \\
Heater outer radius & 0.3 \\
Heater power (Experiment I) & $13.14(\mathrm{~kW})$ \\
RPV inner cavity fluid & vacuum $(1.3 \mathrm{~Pa})$ \\
RPV height & 3.0 \\
RPV inner radius & 0.50 \\
RPV wall thickness & 0.012 \\
RPV top dome inner radius & 0.50 \\
RPV bottom dome major inner radius & 0.50 \\
RPV bottom dome minor inner radius & 0.25 \\
Cavity height & 3.8 \\
Main and lower cavity fluid & air $(1 \mathrm{~atm})$ \\
Cooling tube radial centerline position & 0.9415 \\
Cooling tube outer radius & 0.0318 \\
Cooling tube pitch & 0.06 \\
\hline
\end{tabular}


This task is focused on the first of a number of experiments conducted at the VCS facility. In this particular experiment, the interior of the vessel was maintained at near-vacuum conditions (1.3 Pa). The one-quarter symmetric 3-D mesh used in the model, as shown in Fig. 6, incorporated the heater support, heater support legs, RPV flange, and RPV skirt. A Python script is employed to build the mesh file via the Cubit [25] API.

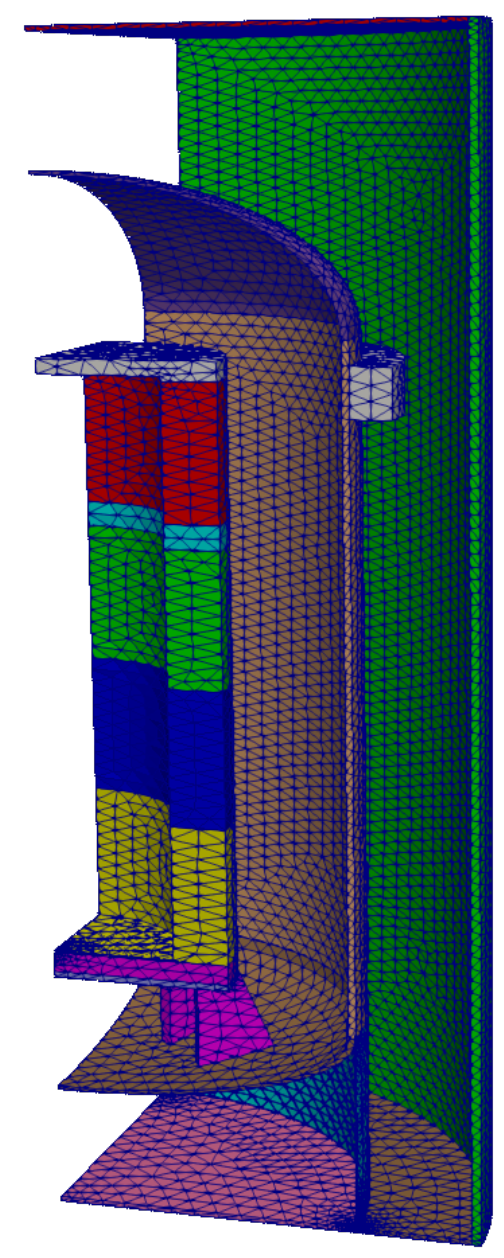

Figure 6: VCS experiment mesh with the cavity volumes removed (1/2 axial scale).

The following assumptions and approximations are adopted:

- The experiment is $1 / 4^{\text {th }}$ symmetric.

- The cooling panels are modeled as a cylindrical or planar surfaces (i.e. the cooling pipes attached to the panels are not explicitly modeled). The position of the radial cooling panel is adjusted to preserve the effective cooling tube surface area in the line-of-sight of the RPV. 
The calculation is performed in the meshing script and the radial position of the cooling tubes in the model is $0.7 \mathrm{~m}$ rather than the $0.9415 \mathrm{~m}$ listed in Table 6 .

- The RPV support legs were not explicitly modeled. Instead, the skirt was extended azimuthally to cover the entire area below the RPV.

- The side and top panels span the full surface area of the side and top walls of the cavity.

- The heater power in each heater segment is equally distributed within the segment using a constant power density value.

All solid materials in the model transfer heat by conduction. The power shown in Table 7 is deposited in each heater block, since the benchmark provides limited information on the heater coil configuration. The water flow in the cooling panels was modeled using a Robin boundary condition with a bulk water temperature of $300 \mathrm{~K}$. Three radiation cavities were included in the model: the inner cavity between the heater and the RPV; the bottom cavity, as defined by the bottom of the RPV, the skirt, and the lower cooling panel; and the main cavity, located between the RPV and the side and top cooling panels. The various heat transfer mechanisms modeled in each cavity are shown in Table 8. Note that natural convection is inhibited inside the RPV, due to the vacuum conditions. Nevertheless, conduction through the low pressure fill-gas plays a non-negligible role via molecular diffusion.

Table 7: Heater segment powers $\left(1 / 4^{\text {th }}\right.$ model $)$.

\begin{tabular}{cccc}
\hline & $\begin{array}{c}\text { Volume } \\
{\left[\mathrm{m}^{3}\right]}\end{array}$ & $\begin{array}{c}\text { Power } \\
{[\mathrm{W}]}\end{array}$ & $\begin{array}{c}\text { Power density } \\
{\left[\mathrm{W} / \mathrm{m}^{3}\right]}\end{array}$ \\
\hline 1 & $4.0780 \mathrm{E}-03$ & $2.5250 \mathrm{E}-01$ & 61917.84585 \\
2 & $1.6596 \mathrm{E}-02$ & $5.7750 \mathrm{E}-01$ & 34797.94542 \\
3 & $1.6596 \mathrm{E}-02$ & $6.6000 \mathrm{E}-01$ & 39767.85473 \\
4 & $1.6596 \mathrm{E}-02$ & $6.1500 \mathrm{E}-01$ & 37056.41247 \\
5 & $1.6757 \mathrm{E}-02$ & $9.4000 \mathrm{E}-01$ & 56095.32563 \\
6 & $4.5881 \mathrm{E}-03$ & $2.4000 \mathrm{E}-01$ & 52309.55111 \\
\hline
\end{tabular}

Natural convection in the bottom and main cavities is modeled via a heat balance with a method consistent to the one described in Section 2.2. Table 9 shows the various parameters used in calculating the heat transfer coefficients. The Rayleigh number depends on the characteristic length $L$ and the temperature gradient through the Grashof number $(\mathrm{Gr})$ since $R a=\mathrm{Gr} \cdot \mathrm{Pr}$. The 
Table 8: Heat transfer mechanisms modeled in each cavity.

\begin{tabular}{lccc}
\hline & conduction & radiation & convection \\
\hline inside RPV & $\mathrm{X}$ & $\mathrm{X}$ & - \\
bottom cavity & - & $\mathrm{X}$ & $\mathrm{X}$ \\
main cavity & - & $\mathrm{X}$ & $\mathrm{X}$ \\
\hline
\end{tabular}

Nusselt numbers for the cavity regions are obtained from the equation for vertical rectangular enclosures, Eq. 21][26]. The Nusselt numbers for horizontal plates (i.e. top and bottom wall regions) are obtained from Eq. 22 [26]. The Nusselt numbers for the RPV dome and ellipsoid are computed with Eq. 23[26].

$$
\begin{gathered}
N u=0.22\left(\frac{P r \cdot R a}{0.2+P r}\right)^{0.28} L^{-\frac{1}{4}} \\
N u=0.15 \cdot R a^{\frac{1}{3}} \\
N u=2+\frac{0.589 \cdot R a^{\frac{1}{4}}}{\left(1+(0.469 / P r)^{\frac{9}{16}}\right)^{\frac{4}{9}}}
\end{gathered}
$$

Table 9: Convective properties for the air cavities. The characteristic length (L) was approximated based on the geometry. $\mathrm{H}$ and $\mathrm{W}$ stand for the height and width of the cavity where natural circulation is established,

\begin{tabular}{lccccccc}
\hline Region & $\begin{array}{c}\text { Equation } \\
\text { for } L\end{array}$ & $\begin{array}{c}L \\
{[m]}\end{array}$ & $\mathrm{Gr}$ & $\mathrm{Pr}$ & $\mathrm{Ra}$ & $\mathrm{Nu}$ & $\begin{array}{c}\mathrm{h} \\
{\left[W / m^{2} / K\right]}\end{array}$ \\
\hline RPV top & $\mathrm{H} / \mathrm{W}$ & 8.85 & $4.55 \mathrm{E}+12$ & 0.705 & $3.21 \mathrm{E}+12$ & 610 & 1.91 \\
RPV side & $\mathrm{H} / \mathrm{W}$ & 8.85 & $5.46 \mathrm{E}+12$ & 0.702 & $3.83 \mathrm{E}+12$ & 397 & 1.29 \\
RPV bottom & numerical & 0.675 & $1.34 \mathrm{E}+09$ & 0.700 & $9.35 \mathrm{E}+08$ & 81.3 & 3.60 \\
skirt side & $\mathrm{H} / \mathrm{W}$ & 8.85 & $2.18 \mathrm{E}+12$ & 0.706 & $1.54 \mathrm{E}+12$ & 307 & 0.95 \\
wall top & $\mathrm{r} / 2$ & 0.471 & $2.02 \mathrm{E}+08$ & 0.705 & $1.43 \mathrm{E}+08$ & 78.4 & 4.61 \\
wall side & $\mathrm{H} / \mathrm{W}$ & 8.85 & $1.95 \mathrm{E}+12$ & 0.702 & $1.37 \mathrm{E}+12$ & 297 & 0.97 \\
wall bottom & $\mathrm{r} / 2$ & 0.250 & $5.24 \mathrm{E}+07$ & 0.700 & $3.67 \mathrm{E}+07$ & 49.8 & 5.96 \\
wall skirt & $\mathrm{H} / \mathrm{W}$ & 8.85 & $9.30 \mathrm{E}+11$ & 0.706 & $6.57 \mathrm{E}+11$ & 242 & 0.75 \\
\hline
\end{tabular}

The radiation heat transfer is modeled using the net radiation transfer method described in Section 2.1. The input includes three GrayDiffuseRadiation cavities and view factors are computed using ray-tracing. The various parameters used in the radiation action are shown in Table 10, It is 
important to note that the current radiation heat transfer module does not allow for temperaturedependent emissivities - which could be important for the heater, since the values for $\mathrm{Al}_{2} \mathrm{O}_{3}$ vary significantly as a function of heater temperature.

Table 10: Radiation heat transfer parameters. The number of directions are per quadrature point. The current radiation heat transfer module does not allow for position-dependent emissivitieswhich could be important for the heater, since the values for $\mathrm{Al}_{2} \mathrm{O}_{3}$ vary significantly as a function of heater temperature.

\begin{tabular}{lccc}
\hline & emissivity & directions & number of patches \\
\hline Heater & 0.70 & 400 & 350 \\
Heater support & 0.79 & 400 & 100 \\
RPV inside & 0.79 & 400 & 450 \\
RPV outside & 0.95 & 400 & 400 \\
RPV bottom & 0.95 & 256 & 150 \\
Cooling tubes (top and side) & 0.95 & 400 & 450 \\
Cooling tubes (bottom) & 0.95 & 256 & 120 \\
\hline
\end{tabular}

The comparisons to the experimental values are shown in Figure 7. The experimental results were obtained from [24] by azimuthally averaging the various thermocouple values in the vicinity of each axial position. Note that the shape of the heater surface temperature is inconsistent with that of the measurement. There appears to be a disagreement in the heater powers or the conduction characteristics of the heater, possibly due to the lack of detail in the position of the heating elements versus the thermocouples. This disagreement needs to be further examined. The shape of the RPV surface temperature agrees pretty well with the measurements, despite the significant uncertainty in the accuracy of the convective heat transfer component of this calculation. The RPV dome temperature is underpredicted in the model, likely due to the low heater temperature at the top of the apparatus. The RPV temperature is overpredicted everywhere else. 


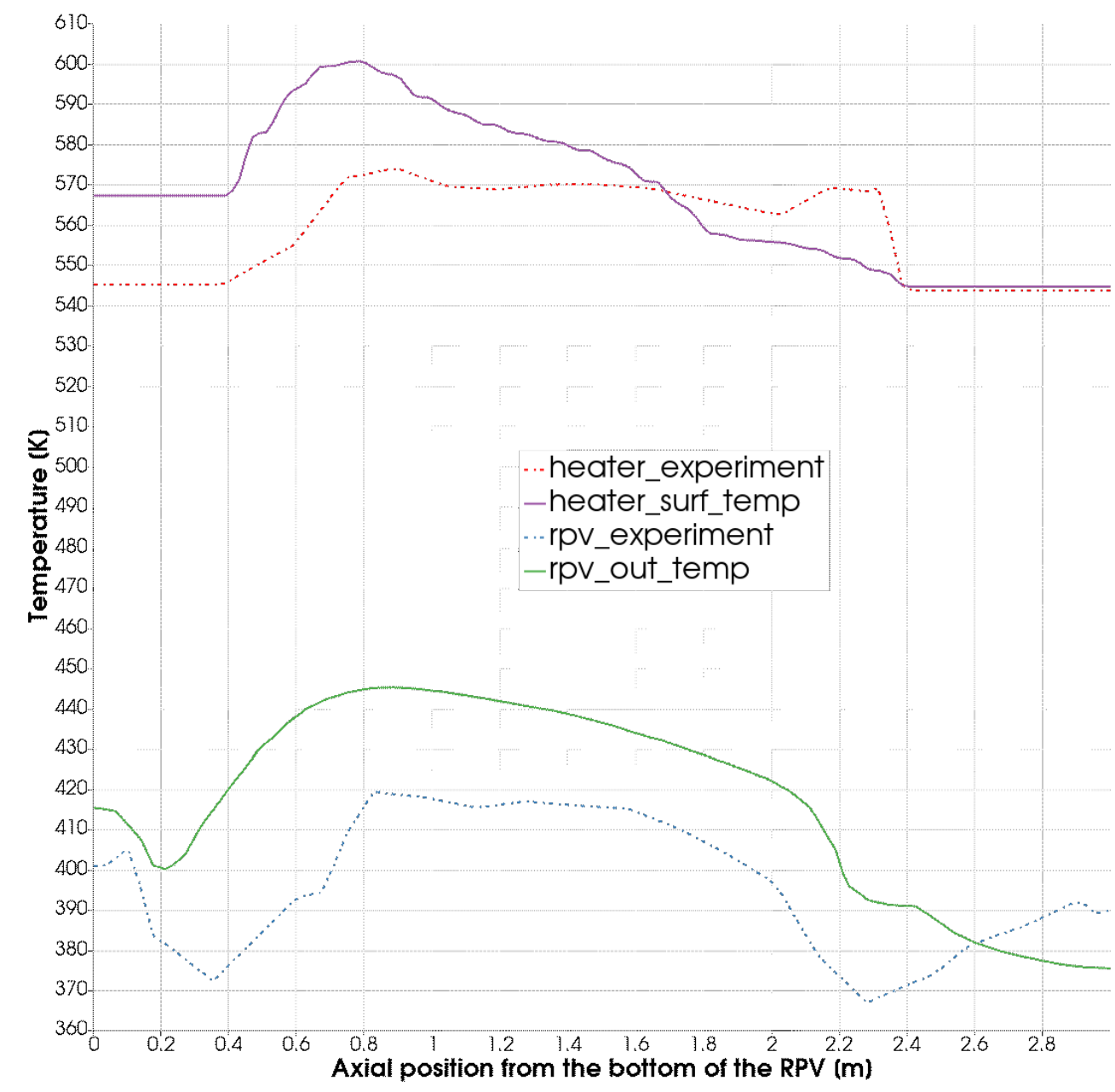

Figure 7: VCS Experiment I results for the heater and RPV temperatures. 


\section{PEBBLE-BED REACTOR EQUILIBRIUM CORE CALCULATION}

This section discusses the initial implementation of pebble depletion and shuffling into the reactor physics code Griffin [27]. Pebble depletion is the computation of the isotopic composition of pebbles as they move through the core, are recirculated from the core outlet to the core inlet, and finally disposed of. Pebble shuffling is a subtask within the pebble depletion computation. The pebble shuffling routine tracks pebbles (not isotopic content) of a certain type as they move along streamlines through the core and are mixed at the core outlet, separated into discarded and recirculated pebbles, mixed with fresh pebbles, and finally reloaded into the core. The task of pebble depletion requires capabilities in addition to pebble shuffling, including neutron and gamma flux solvers, "online" cross-section generation, transmutation, and a decay solver. A pebble shuffling capability is necessary for pebble depletion calculations. Pebble motion in a pebble-bed reactor is modeled as an incompressible fluid; it is not customary to follow each pebble through its entire lifecycle.

The two relevant operational scenarios for pebble depletion are equilibrium core calculations and running-in calculations. The equilibrium core is the steady-state distribution of the various fields (isotopic, neutron/gamma flux, temperature, etc.), which is approached after operating the reactor for a sufficient duration under a consistent refueling policy (i.e., problem constraints). The running-in is the transient period that leads from a non-asymptotic core (usually the initial critical core) to the equilibrium core. The isotopic composition and related fields of the core during the running-in depend on time, whereas it is time-independent in the equilibrium core state. This report only describes the implementation of an equilibrium core depletion capability into Griffin.

The methods described here and the current implementation in Griffin are preliminary. The equilibrium core capability has not been thoroughly verified against reference solutions; therefore, the results presented in this section are subject to change as testing on this capability continues.

\subsection{Outline of the Equilibrium Core Depletion Algorithm}

The approach for modeling pebble depletion in gas-cooled pebble-bed reactors (PBRs) and fluoridesalt-cooled pebble-bed reactors (FHRs) is explained based on the conceptual sketch depicted in Fig. 8. This sketch resembles a PBR refueling scheme, and the essence of the FHR refueling scheme 
is identical, except that the pebbles traverse the core from the bottom up. However, this difference does not significantly change the modeling approach.

The flow of pebbles through the PBR and FHR cores is essentially 1-D; pebbles follow streamlines from the inlet to the outlet, and a pebble that enters a particular streamline at the pebble inlet does not leave that streamline until it leaves the core at the exit of the defueling chute [28]. The active part of the core is referred to as the pebble bed, while the low-power/no-power section is referred to as the defueling chute. The streamlines through the core are indexed by $k=1, \ldots, K$. We measure the distance along the streamline using the variable $s$.

All streamlines merge at the outlet of the defueling chute, and pebbles move from the defueling chute to the pebble handling and storage system (PHSS). The PHSS is represented as a dashed box at the top of the core in Fig. 8. In the sketch, the physical location of the PHSS is purely conceptual, and it is not implied that all the indicated operations occur in a singe location on top of the core. We conceptualize the PHSS as a single control volume that performs the following operations:

- Merging of streamlines

- Tracer isotope measurement and the decision to discard pebbles above the burnup threshold

- Adding fresh fuel pebbles to replace discarded ones

- Distributing pebbles over streamlines at the core inlet.

At the outlet of the defueling chute, the pebbles' radiation signature is measured to determine their burnup. Pebbles with burnups exceeding the limit are discarded, while pebbles below that threshold are recirculated. The recirculated pebbles are mixed with fresh pebbles then distributed among the streamlines, following an operator-defined recirculation strategy.

This report lays out balance equations for tracking pebbles and isotopes as they are irradiated while moving through the pebble-bed reactor. The following assumptions are adopted throughout this report:

- The PHSS cannot store pebbles, but pebbles spend a non-negligible time outside the core.

- Pebbles move on streamlines so that the problem of pebble depletion is essentially 1-D in a spatial sense. 


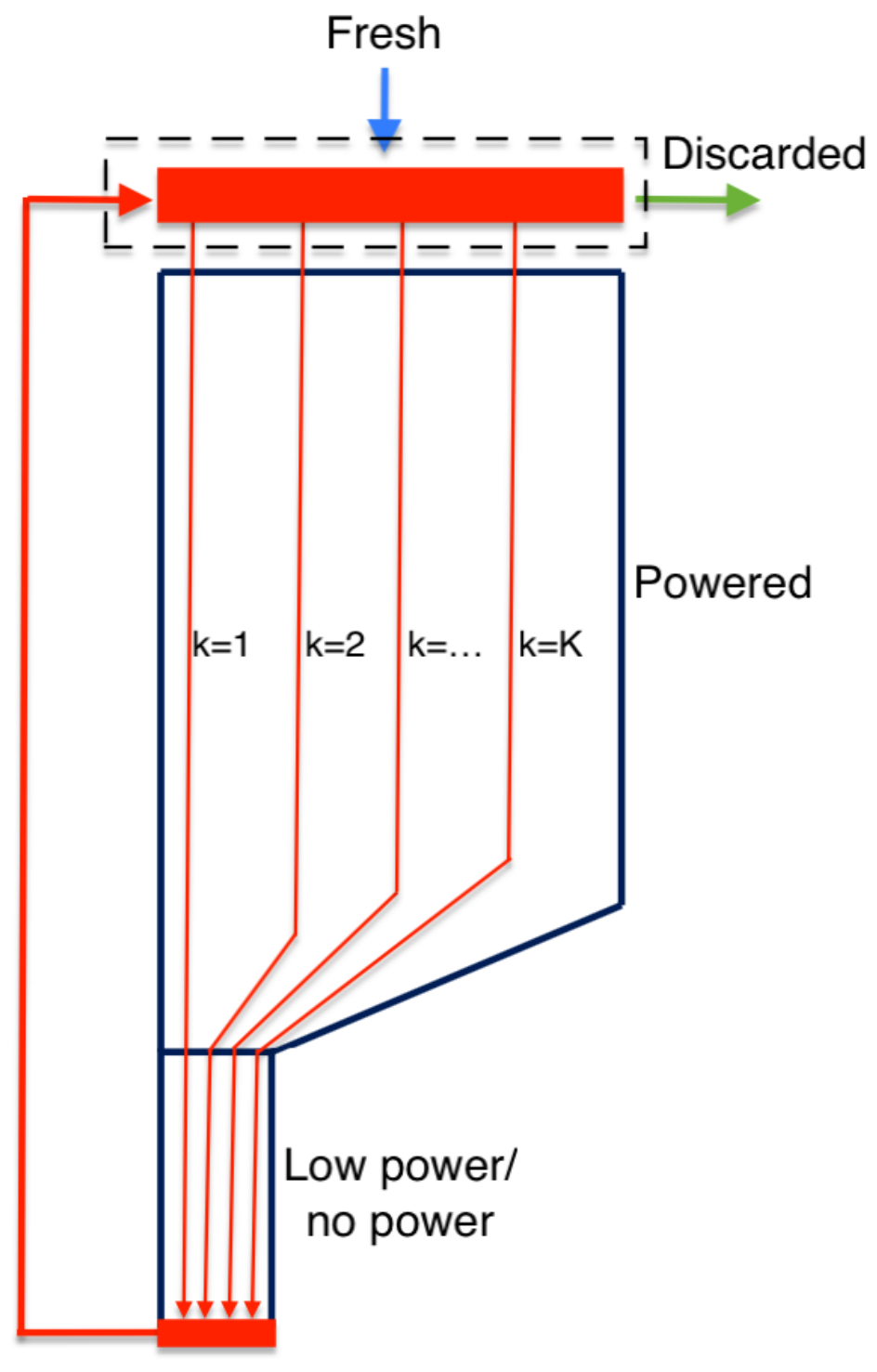

Figure 8: Concept of the pebble recirculation scheme. The sketch serves as basis for the modeling approach adopted in Griffin. 


\subsection{Governing Equations}

This section introduces balance relations for pebbles and isotopes over streamlines.

\subsubsection{Streamlines}

A streamline is the path traced out by a pebble as it moves through the core vat. Two pebbles that enter the core vat close to each other remain close as they move though the core; their streamlines do not diverge significantly. Therefore, pebble motion can be described accurately by solving the relevant equations (derived later in this section) for a sufficient number of representative streamlines. This reduces the problem from a multi-dimensional problem to a finite number of 1-D problems.

Instead of envisioning streamlines as infinitely thin curves through the reactor core, it is useful to think of them as having a finite extent perpendicular to the flow direction (i.e., we associate an area to the streamline perpendicular to the streamline's direction). In this way, the reactor can be divided into streamtubes. Streamtubes can take the shape of radial sectors or concentric rings, as outlined in Ref. [29]. Despite having a finite extent, this document refers to these streamtubes as "streamlines."

Pebble streamlines are indexed by subscript $k$. The location on any given streamline is uniquely identified by the distance along streamline $s$ from the inlet of the streamline (i.e., the location where pebbles enter the streamline). The mapping from streamline index $k$ and distance along streamline $s$ to a point in space is denoted by $\vec{r}_{k}(s)$.

\subsubsection{Balance Equation for Pebbles on Streamlines}

The fraction of volume occupied by pebbles on streamline $k$ at position $s$ is denoted by $n_{k}(s)$.

$$
n_{k}(s)=\frac{\text { Volume occupied by pebbles }}{\text { Total volume }}
$$

The definition on the right-hand side of Eq. 24 is to be understood as follows: at location $s$ along streamline $k$, a representative volume centered at $s$ is selected that contains a sufficient number of pebbles. The total extent of this representative volume is the denominator of Eq. 24, while the 
portion occupied by the pebbles is the numerator. Both the numerator and denominator on the right-hand side of Eq.24 depend on s, and index $k$, but we omit these details for the sake of brevity. We treat $n_{k}(s)$ as a continuous function, implying that $n_{k}(s)$ can be meaningfully evaluated at a given point, even though the definition of $n_{k}(s)$ requires a representative volume of finite size.

The fraction of pebble volumes is related to the porosity by $n_{k}(s)=1-\epsilon_{k}(s)$. The steady-state balance equation for $n_{k}(s)$ is as follows:

$$
u_{k}(s) A_{k}(s) n_{k}(s)=u_{k}(s) A_{k}(s)\left(1-\epsilon_{k}(s)\right)=\text { constant along streamline }=\dot{n}_{k}
$$

where $u_{k}(s)$ is the speed of pebbles tangential to streamline $k$ at location $s, A_{k}(s)$ is the crosssectional area of streamline $k$ at distance $s$ from the streamline inlet, and $\dot{n}_{k}$ is the volumetric flow rate of pebbles on streamline $k$ (i.e., the volume of all pebbles introduced into the streamline, divided by time). These equations allow for computing the speed of pebbles $u_{k}(s)$ for any location along the streamline via the streamline geometry, local porosity, and rate at which pebbles are introduced into the streamline at the pebble inlet.

In steady-state, the volumetric flow rate at which pebbles are unloaded from the core and enter the PHSS, $\dot{n}_{u}$, is computed by:

$$
\dot{n}_{u}=\left.\sum_{k=1}^{K} u_{k} A_{k} n_{k}\right|_{s_{k, e}}
$$

where $s_{k, \mathrm{e}}$ is the coordinate of the exit of streamline $k$. The rate $\dot{n}_{u}$ is directly determined by the operator. From discrete element studies, it is well-established that "for dense granular flows in the quasi-static regime, the overall flow rate has a weak effect on the features of flow" [30]. This implies that $\dot{n}_{k} / \dot{n}_{u}=$ const. $=\xi_{k}$. The relative distribution of volumetric flow rates among streamlines in the pebble bed is independent of the total drainage rate and determined solely by the geometry of the pebble-bed reactor and the pebbles.

\subsubsection{Measuring Pebble Burnup}

The accumulated pebble burnup is tracked so that pebbles exceeding the maximum burnup can be discarded at the outlet of the defueling chute. In practice, burnup is determined by measuring the concentration of tracer isotopes. In pebble burnup simulations, the number of passes through 
the core is traditionally used to determine when a pebble is removed from recirculation; every pebble makes $L$ passes and is not recirculated for a $L+1$-th pass. The number of passes correlates to the amount of burnup a pebble has experienced. However, depending on the exact path of the pebble during each pass through the core, two pebbles with $L$ passes through the core can have different burnups.

We propose to group pebbles directly according to the accumulated burnup $\tau$. To this end, we define $n_{k}(s, \tau)$ as the distribution of pebble volume fraction over burnup:

$$
n_{k}(s, \tau) d \tau=\frac{\left[\begin{array}{l}
\text { Volume occupied by a pebbles on streamline } k \\
\text { with burnup between } \tau \text { and } \tau+d \tau
\end{array}\right]}{\text { Total volume }}
$$

where we again stress that both the numerator and denominator on the right-hand side depend on $s$. We define the evolution operator of a distribution over burnup by:

$$
\mathbf{T}[\cdot]=A_{k}(s) \frac{\partial\left[p\left(\vec{r}_{k}(s), \tau\right) \cdot\right]}{\partial \tau}
$$

where $p\left(\vec{r}_{k}(s), \tau\right)$ is the power density experienced by a pebble population with a burnup between $\tau$ and $\tau+d \tau$. The operator, $\mathbf{T}$, is totally equivalent to the operator advecting a scalar quantity in space: burnup, $\tau$, takes on the role of spatial position, and power density, $p$, takes on the role of advection speed.

The power experienced by a group of pebbles significantly depends on burnup, since the isotopic composition depends strongly on burnup [29]. Groups of pebbles with different burnups that coexist at a given location share the same average neutron flux. However, as the isotopic content of these groups of pebbles is different, the power they experience differs. The pebble power density is computed by:

$$
p\left(\vec{r}_{k}(s), \tau\right)=\int_{0}^{\infty} \kappa \Sigma_{f}(\vec{r}, E, \tau) \mathbf{P}_{k}[\phi(\vec{r}, E)] d E
$$

where $\kappa \Sigma_{f}(\vec{r}, E, \tau)$ is the macroscopic fission heating cross section for pebbles with burnups between $\tau$ and $\tau+d \tau, \phi(\vec{r}, E)$ is the neutron flux of neutrons with energies between $E$ and $E+d E$, 
and $\mathbf{P}_{k}$ is an operator that averages the flux at $\vec{r}$ over the extent of the streamline at $\vec{r}_{k}(s)$. A formula for computing $\kappa \Sigma_{f}(\vec{r}, E, \tau)$ is provided later.

We divide the burnup range into $L$ intervals and denote the end points of each burnup range by $\left[\tau_{l}, \tau_{l+1}\right]$ with $\tau_{1}=0$ and $\tau_{L}=\tau_{\max }$, where $\tau_{\max }$ is the burnup limit for pebble discharge so that all pebbles in the $L$-th group are discharged once they complete their current pass through the core. Finally, $\tau_{L+1}$ must be large enough that it can never be exceeded.

Grouping pebbles by burnup is an alternative to grouping them by number of passes. Both options will be pursued in implementing the pebble depletion algorithm.

\subsubsection{Balance Equation for Pebble Types on Streamlines}

We denote the volume fraction occupied by a pebble of type $c$, as identified by its initial composition on streamline $k$, by $n_{c, k}$. The volume fraction $n_{c, k}(s, \tau)$ depends on the distance $(s)$ measured along the streamline from the core inlet, and on burnup $(\tau)$ :

$$
n_{c, k}(s, \tau) d \tau=\frac{\left[\begin{array}{l}
\text { Volume occupied by a pebble of type } c \\
\text { identified by initial composition on streamline } k \\
\text { with burnup between } \tau \text { and } \tau+d \tau
\end{array}\right]}{\text { Total volume }}
$$

The relationship between $n_{c, k}(s, \tau)$ and $n_{k}(s)$ is:

$$
n_{k}(s)=\sum_{c=1}^{C} \int_{0}^{\infty} n_{c, k}(s, \tau) d \tau
$$

As a short-hand, we will refer to pebble type $c$ (identified by its initial composition) as simply "type c."

The balance equation for $n_{c, k}(s, \tau)$ along the streamline is given by:

$$
\begin{aligned}
& \frac{\partial\left(u_{k}(s) A_{k}(s) n_{c, k}(s, \tau)\right)}{\partial s} \\
& +A_{k}(s) \frac{\partial\left(p_{c}\left(\vec{r}_{k}(s), \tau\right) n_{c, k}(s, \tau)\right)}{\partial \tau}=0
\end{aligned}
$$

where $p_{c}\left(\vec{r}_{k}(s), \tau\right)$ is the power density that a pebble of type $c$ with a burnup between $\tau$ and $\tau+d \tau$ 
experiences.

The fraction of volume occupied by pebbles of type $c$ with burnups falling into group $l$ is given by:

$$
n_{c, k, l}(s)=\int_{\tau_{l}}^{\tau_{l+1}} n_{c, k}(s, \tau) d \tau
$$

Integrating Eq. 32 over $\tau$ within $\left[\tau_{l}, \tau_{l+1}\right]$ gives:

$$
\begin{aligned}
& \frac{\partial\left(u_{k} A_{k} n_{c, k, l}(s)\right)}{\partial s} \\
& +A_{k}\left[p_{c}\left(\tau_{l+1}\right) n_{c, k}\left(\tau_{l+1}\right)-p_{c}\left(\tau_{l}\right) n_{c, k}\left(\tau_{l}\right)\right]=0
\end{aligned}
$$

where obvious arguments are omitted for convenience of notation. We approximate $p_{c}\left(\tau_{l}\right) n_{c, k}\left(s, \tau_{l}\right)$ using upwinding in the direction of increasing burnup:

$$
p_{c}\left(\tau_{l}\right) n_{c, k}\left(\tau_{l}\right) \approx \begin{cases}0 & \text { if } l=1 \\ 0 & \text { if } l=L+1 \\ \frac{\bar{p}_{c, l-1} n_{c, k, l-1}}{\Delta \tau_{l-1}} & \text { else }\end{cases}
$$

where $\Delta \tau_{l}=\tau_{l+1}-\tau_{l}$ and the average power is defined by:

$$
\bar{p}_{c, l}\left(\vec{r}_{k}(s)\right)=\frac{1}{\Delta \tau_{l}} \int_{\tau_{l}}^{\tau_{l+1}} p_{c}\left(\vec{r}_{k}(s), \tau\right) d \tau
$$

This result leads to the semi-discrete form of Eq. 34 .

$$
\frac{\partial\left(u_{k} A_{k} n_{c, k, l}\right)}{\partial s}+A_{k}\left[\frac{\bar{p}_{c, l} n_{c, k, l}}{\Delta \tau_{l}}-\frac{\bar{p}_{c, l-1} n_{c, k, l-1}}{\Delta \tau_{l-1}}\right]=0
$$

This equation couples the population of pebbles in burnup group $l$ and at location $s$ to the neighboring burnup group $l-1$ and neighboring locations in space. When discretizing the derivative with respect to $\tau$, we used a constant approximation of power over each burnup group (i.e., $\left.p_{c}\left(\vec{r}_{k}(s), \tau_{l}\right) \approx \bar{p}_{c, l}\left(\vec{r}_{k}(s)\right)\right)$. The computation of the average power will be discussed in Sec. 3.2.5 because it requires the definition of isotope concentrations in pebbles.

If burnup is measured by the number of passes a pebble has experienced, the last term is 
simply omitted, and we obtain a simple advection equation along the streamline:

$$
\frac{\partial\left(u_{k} A_{k} n_{r, k, l}\right)}{\partial s}=0
$$

The index $l$ remains unchanged since pebbles move on streamlines, and would only increase when pebbles leave the core and enter the PHSS.

\subsubsection{Balance Equation for Isotopes on Streamlines}

We denote the concentration of isotope $q$ contained in a pebble of type $c$ traversing the core on streamline $k$ by $N_{q, c, k}$. The concentration $N_{q, c, k}(s, \tau)$ depends on the distance $(s)$ measured along the streamline from the core inlet, and on burnup $(\tau)$ :

$$
N_{q, c, k}(s, \tau) d \tau=\frac{\left[\begin{array}{l}
\text { Number of atoms of isotope } q \text { in pebbles with initial composition } c \\
\text { on streamline } k \text { with burnup between } \tau \text { and } \tau+d \tau
\end{array}\right]}{\text { Total volume }}
$$

It is convenient to collect the different isotopes in the vector $\vec{N}_{c, k}$ that contains the concentration $N_{q, c, k}$ as its $q^{\text {th }}$ entry.

The balance equation for $\vec{N}_{c, k}(s, \tau)$ along the streamline is given by:

$$
\frac{1}{A_{k}(s)} \frac{\partial\left(u_{k} A_{k} \vec{N}_{c, k}\right)}{\partial s}+\frac{\partial\left(p_{c}(\tau) \vec{N}_{c, k}\right)}{\partial \tau}=\mathbf{A}(\phi) \vec{N}_{c, k}(s, \tau)
$$

where $\mathbf{A}(\phi)$ is the transmutation and decay matrix that depends on the neutron flux $(\phi)$. The entries in the transmutation and decay matrix are:

$$
\begin{aligned}
& \mathbf{A}_{q, q}=-\lambda_{q}-\bar{\sigma}_{a, q} \bar{\phi} \\
& \mathbf{A}_{q, m}=\lambda_{m} \gamma_{m \rightarrow q}^{d}+\bar{\sigma}_{a, m} \gamma_{m \rightarrow q}^{a} \bar{\phi}
\end{aligned}
$$

where $\lambda_{q}$ is the decay constant of isotope $q, \bar{\sigma}_{a, q}$ is the collapsed microscopic neutron disappearance cross section of isotope $q, \bar{\phi}$ is the collapsed one-group scalar flux, $\gamma_{m \rightarrow q}^{d}$ is the fraction of decays of isotope $m$ leading to the creation of isotope $q$ (i.e., the branching ratio), and $\gamma_{m \rightarrow q}^{a}$ is the 
fraction of neutron disappearance reactions for isotope $m$ leading to the creation of isotope $q$.

Analogous to $n_{c, k, l}$, we define $\vec{N}_{c, k, l}$ as follows:

$$
\vec{N}_{c, k, l}=\int_{\tau_{l}}^{\tau_{l+1}} \vec{N}_{c, k}(s, \tau) d \tau
$$

Integrating Eq. 40 and using the same discretization as for Eq. 37 leads to:

$$
\frac{1}{A_{k}(s)} \frac{\partial\left(u_{k} A_{k} \vec{N}_{c, k, l}\right)}{\partial s}+\left[\frac{\bar{p}_{c, l} \vec{N}_{c, k, l}}{\Delta \tau_{l}}-\frac{\bar{p}_{c, l-1} \vec{N}_{c, k, l-1}}{\Delta \tau_{l-1}}\right]=\mathbf{A} \vec{N}_{c, k, l}
$$

The average power, $\bar{p}_{c, l}$, remains to be defined:

$$
\left.\bar{p}_{c, l}\left(\vec{r}_{k}(s)\right)=\sum_{q=1}^{Q} \frac{N_{q, c, k, l}}{n_{c, k, l}} \int_{0}^{\infty} \kappa \sigma_{f, q}\left(E, \mathbf{P}_{k}[T(\vec{r})]\right)\right) \mathbf{P}_{k}[\phi(\vec{r}, E)] d E
$$

where $T(\vec{r})$ is the temperature. The details regarding Eq. 44 require some explanation. The number density $N_{q, c, k, l}$ is the number of atoms of type $q$ divided by the total volume. However, to compute the average power density in pebbles of type $c$ within burnup groups $l$, we need to use the isotope number density computed using the volume of pebble type $c$ and burnup group $l$ (as opposed to the number densities with respect to the total volume), as given by $N_{q, c, k, l} / n_{c, k, l}$. The average pebble power density, $\bar{p}_{c, l}$, is related to the average reactor power density by:

$$
p(\vec{r})=\sum_{c, l} n_{c, k, l} \bar{p}_{c, l}\left(\vec{r}_{k}(s)\right)=\sum_{q=1}^{Q} N_{q, k}(s) \int_{0}^{\infty} \kappa \sigma_{f, q}(E) \phi\left(\vec{r}_{k}(s), E\right) d E
$$

which is a weighted average over the different pebble types. Note that $p(\vec{r})$ is the power density with respect to the entire volume (i.e., total power divided by reactor volume not pebble volume).

\subsubsection{Balance Equation for Pebble Types in the PHSS}

The PHSS provides the boundary conditions for Eqs. 37 and 43 . We model the PHSS as a single control volume. The control volume is assumed to be small; consequently, pebbles cannot be stored in it. This implies that the time derivative of both pebble content and isotopic content is zero. Therefore, the instantaneous flow of pebbles into the PHSS equals the instantaneous flow out 
of the PHSS. These assumptions do not preclude us from accounting for the effect of the residence time in the PHSS on the nuclide concentration via radioactive decay.

To specify boundary conditions for Eq. 37, we need to compute $\dot{n}_{c, k, l}$, defined as the volumetric flow rate of pebbles of type $c$ and burnup group $l$ reloaded into streamline $k$. The balance equation for the PHSS is given by:

$$
\begin{aligned}
0= & \sum_{k=1}^{K}\left[A_{k} u_{k}\left(s_{k, \mathrm{e}}\right) n_{c, k, l-p}\left(s_{k, \mathrm{e}}\right)\left(1-\beta_{c, k, l}\right)-\dot{n}_{c, k, l}\right]+\dot{n}_{c, F} \delta_{l, 1}=0 \\
& \text { for } l=1, \ldots, L-1 ; c=1, \ldots, C \\
\dot{n}_{D}= & \dot{n}_{D, s}+\dot{n}_{D, d} \\
\dot{n}_{D, s}= & \sum_{c=1}^{C} \sum_{k=1}^{K} A_{k} u_{k}\left(s_{k, \mathrm{e}}\right) n_{c, k, L-p}\left(s_{k, \mathrm{e}}\right) \\
\dot{n}_{D, d}= & \sum_{l=1}^{L-1} \sum_{c=1}^{C} \sum_{k=1}^{K} A_{k} u_{k}\left(s_{k, \mathrm{e}}\right) n_{c, k, l-p}\left(s_{k, \mathrm{e}}\right) \beta_{c, k, l}
\end{aligned}
$$

where $\delta_{i, j}$ is the Kronecker delta, $p=1$ if we use number of passes as measure of burnup (otherwise, $p=0), \dot{n}_{c, F}$ is the volumetric flow rate of fresh pebbles of type $c, \beta_{c, k, l}$ is the fraction of pebbles of type $c$ and burnup group $l$ that leave streamline $k$ sufficiently damaged for the pebbles to be discarded, $\dot{n}_{D}$ is the volumetric flow rate of discarded pebbles, $\dot{n}_{D, s}$ is the volumetric flow rates of pebbles that are discarded because they exceed the burnup threshold, and $\dot{n}_{D, d}$ is the volumetric flow rate of pebbles that are discarded because of damage.

The volumetric flow rate at which new pebbles are loaded into the core is denoted by $\dot{n}_{F}$ and is given as the sum over all $\dot{n}_{c, F}$ :

$$
\dot{n}_{F}=\sum_{c=1}^{C} \dot{n}_{c, F}
$$

For the equilibrium core calculation, the total number of pebbles in the core must remain constant, hence:

$$
\dot{n}_{F}=\dot{n}_{D}
$$

Eq. 48 does not provide any condition on the pebble types to be loaded. The fraction of fresh pebbles of type $c$, denoted by $\chi_{c, F}$, is set by the operator and is therefore an input parameter for 
the algorithm. The volumetric flow rate of fresh pebbles of type $c$ is then given by:

$$
\dot{n}_{c, F}=\chi_{c, F} \dot{n}_{F}
$$

We now consider the distribution of pebbles recirculated into the core over the different streamlines. The total volumetric flow rate of pebbles of type $c$ and burnup group $l$ reloaded into the core is:

$$
\dot{n}_{c, l}=\sum_{k=1}^{K}\left[A_{k} u_{k}\left(s_{e}\right) n_{c, k, l-p}\left(s_{e}\right)\left(1-\beta_{c, k, l}\right)\right]+\dot{n}_{c, F} \delta_{l, 1}
$$

The ratio of volumetric flow rates in each channel, as well as the total volumetric flow rate of pebbles through the core, is constant. Therefore, the distribution of the total flow rate on the $K$ flow channels must follow:

$$
\dot{n}_{c, k, l}=\xi_{k} Y_{k, l} \dot{n}_{c, l}
$$

where $Y_{k, l}$ encapsulates the zoning strategy. Zoned cores distribute the pebbles into different streamlines, depending on their burnup. The zoning strategy is supplied by the operator and represents an important control on the recirculation policy.

The zoning policy must satisfy several normalization conditions. First, summing over $c$ gives:

$$
\dot{n}_{k, l}=\xi_{k} Y_{k, l} \dot{n}_{l}
$$

The first set of normalization conditions is obtained by summing over flow channels $k$ :

$$
\sum_{k=1}^{K} \dot{n}_{k, l}=\dot{n}_{l}=\left(\sum_{k=1}^{K} \xi_{k} Y_{k, l}\right) \dot{n}_{l} \rightarrow\left(\sum_{k=1}^{K} \xi_{k} Y_{k, l}\right)=1 \text { for } l=1, \ldots, L
$$

The second set of normalization conditions is obtained by summing over all burnup groups:

$$
\sum_{l=1}^{L} \dot{n}_{k, l}=\dot{n}_{k}=\frac{\dot{n}_{k}}{\dot{n}_{u}}\left(\sum_{l=1}^{L} Y_{k, l} \dot{n}_{l}\right) \rightarrow\left(\sum_{l=1}^{L} \zeta_{l} Y_{k, l}\right)=1 \text { for } k=1, \ldots, K
$$

where $\zeta_{l}=\dot{n}_{l} / \dot{n}_{u}$. Currently being investigated is how to ensure proper normalization of $Y_{k, l}$ and closely relate it to the information available to plant designers and operators. Note that the "random" recirculation given by $Y_{k, l}=1 / K$ naturally satisfies the normalization conditions. 
Summing $\dot{n}_{c, k, l}$ over all flow channels leads to the following constraint on $Y_{k, l}$ :

$$
\sum_{k=1}^{K} Y_{k, l}=1
$$

Solving Eq. 46 for $\dot{n}_{c, k, l}$ and substituting intermediate results leads to an explicit equation:

$$
\dot{n}_{c, k, l}=\xi_{k} Y_{k, l}\left(\sum_{k=1}^{K}\left[A_{k} u_{k}\left(s_{e}\right) n_{c, k, l-p}\left(s_{e}\right)\left(1-\beta_{c, k, l}\right)\right]+\chi_{c, F} \dot{n}_{F}\right)
$$

The operator can manipulate the recirculation policy of the pebble-bed reactor through various means. The roles of the parameters in Eq. 56 are explained in Table 11.

Table 11: Parameters for modeling the PHSS and their role.

\begin{tabular}{lll}
\hline Parameter & Name & Type of parameter \\
\hline$\xi_{k}$ & $\begin{array}{l}\text { Distribution of pebble } \\
\text { flow rates over streamlines }\end{array}$ & $\begin{array}{l}\text { Determined by core \& pebble geometry, } \\
\text { computed by DEM }\end{array}$ \\
$\chi_{c, F}$ & $\begin{array}{l}\text { Fraction of pebble type } c \\
\text { in fresh pebble feed }\end{array}$ & Operator specified \\
$\dot{n}_{F}$ & Fresh pebble feed rate & $\begin{array}{l}\text { Running-in -perator specified } \\
\text { Eq. core: } \dot{n}_{F}=\dot{n}_{D}\end{array}$ \\
$\beta_{c, k, l}$ & $\begin{array}{l}\text { Fraction of pebbles leaving } \\
\text { the core damaged }\end{array}$ & $\begin{array}{l}\text { Operational parameter, } \\
\text { needs to be estimated or provided }\end{array}$ \\
$Y_{k, l}$ & $\begin{array}{l}\text { Zoning of pebbles into } \\
\text { streamline } k \text { by burnup }\end{array}$ & Operator specified \\
\hline
\end{tabular}

\subsubsection{Balance Equation for Isotopes in PHSS}

It is assumed that the PHSS cannot store pebbles or an isotopic inventory. However, it is important that the decay of isotopes occurring within the PHSS is accounted for. We denote the time that a pebble spends in the PHSS by $\Delta t_{\text {phss }}$. The balance equation for the isotope concentration is then given by:

$$
0=\sum_{k=1}^{K}\left[A_{k} u_{k} \exp \left(\mathbf{A}(0) \Delta t_{p h s s}\right) \vec{N}_{c, k, l-p}\left(1-\beta_{c, k, l}\right)\right]_{s_{e, k}}-\dot{\vec{N}}_{c, l}+\dot{n}_{c, F} \vec{C}_{c} \delta_{l, 1}
$$


where $\mathbf{A}(0)$ is the radioactive decay matrix, $\dot{\vec{N}}_{c, l}$ is the volumetric flow rates of atoms of type $q$ contained in pebbles of type $c$ and current burnup group $l$ reloaded into the core, and $\vec{C}_{c}$ is defined component-wise as follows:

$$
\left(\vec{C}_{c}\right)_{q}=\frac{\text { Number of atoms of isotope } q \text { in a fresh pebble of type } c}{\text { Volume of pebble type } c}
$$

The atom flow rate, $\dot{\vec{N}}_{c, l}$, is divided among the streamlines in the same proportion as the pebbles:

$$
\dot{\vec{N}}_{c, k, l}=\xi_{k} Y_{k, l} \dot{\vec{N}}_{c, l}
$$

Using Eqs. 59, 57, and 49 , we obtain an explicit expression for $\dot{\vec{N}}_{c, k, l}$ :

$$
\dot{\vec{N}}_{c, k, l}=\xi_{k} Y_{k, l}\left(\sum_{k=1}^{K}\left[A_{k} u_{k} \exp \left(\mathbf{A}(0) \Delta t_{p h s s}\right) \vec{N}_{c, k, l-p}\left(1-\beta_{c, k, l}\right)\right]_{s_{e, k}}+\chi_{c, F} \vec{C}_{c} \dot{n}_{F}\right)
$$

\subsection{Solution Algorithm and Data Transfers}

This section discusses how the pebble volume fraction balance equation (Eq. 37 ) and the nuclide density balance equation (Eq. 43) are subject to periodic boundary conditions (Eqs. 56, and 60, respectively). The equations are solved iteratively. At each iteration, the inflow boundary conditions are evaluated from the computed outflow of the previous iteration. When the inflow boundary conditions are given, the solution of Eqs. 37 and 43 can be obtained by "sweeping" along the streamline from inlet to outlet and, at each element, from lowest to highest burnup group. For each element and burnup group, the pebble volume fraction is obtained by a simple algebraic equation, while the nuclide densities are obtained by solving a linear system of equations that couples all nuclide densities of a single burnup group.

Each streamline is divided into $m=1, \ldots, M_{k}$ elements, and the boundary between element $m$ and $m+1$ is denoted by $m+1 / 2$. The spatial derivative in Eqs. 37 and 43 is discretized using a simple upwind scheme:

$$
\left.\frac{\partial u_{k} A_{k} n_{c, k, l}}{\partial s}\right|_{s_{m}} \approx u_{k, m} A_{k, m} n_{c, k, l, m}-u_{k, m-1} A_{k, m-1} n_{c, k, l, m-1}
$$

where $u_{k, m}$ is computed from Eq. 25 at the midpoint of element $m, A_{k, m}$ is the streamline area at the 
midpoint of element $m$, and $n_{c, k, l, m}$ is the average of $n_{c, k, l}$ over element $m$. The solution algorithm described in this section can accommodate other differencing schemes without major modifications if they only use upwind information; if downwind information is required, then deferred correction can be used to enable sweeping. The spatial derivative of the isotope concentration is discretized in exactly the same way.

Substituting Eq. 61 into Eq. 37 and rearranging gives:

$$
n_{c, k, l, m}=\frac{u_{k, m-1} A_{k, m-1} n_{c, k, l, m-1}+\frac{\bar{p}_{c, l-1, m} A_{k, m}}{\Delta \tau_{l-1}} n_{c, k, l-1, m}}{u_{k, m} A_{k, m}+\frac{\bar{p}_{c, l, m} A_{k, m}}{\Delta \tau_{l}}}
$$

where $\bar{p}_{c, l, m}$ is $\bar{p}_{c, l}$ either averaged over element $m$ or evaluated at the midpoint of element $m$ (the distinction matters only for higher-order discretization schemes). For the nuclide balance equation (Eq. 43), we obtain:

$$
\left[\left(u_{k, m} A_{k, m}+\frac{\bar{p}_{c, l, m} A_{k, m}}{\Delta \tau_{l}}\right) \mathbf{I}-\mathbf{A}\right] \vec{N}_{c, k, l, m}=u_{k, m-1} A_{k, m-1} \vec{N}_{c, k, l, m-1}+\frac{\bar{p}_{c, l-1, m} A_{k, m}}{\Delta \tau_{l-1}} \vec{N}_{c, k, l-1, m}
$$

Using Eqs. 62 and 63 , the solution is obtained by sweeping burnup groups and elements in the direction of increasing $l$ and $m$ indices.

The set of equations consisting of Eqs. 37, 43, 56, and 60 is homogeneous. Therefore, its solution is determined only up to a scaling factor. To obtain a unique solution, we enforce the following normalization:

$$
\sum_{l=1}^{L} \sum_{c=1}^{C} \sum_{k=1}^{K} A_{k} u_{k}\left(s_{k, \mathrm{e}}\right) n_{c, k, l}\left(s_{k, \mathrm{e}}\right)=\dot{n}_{u}
$$

This normalization ensures that the following condition is satisfied:

$$
\sum_{l=1}^{L} \sum_{c=1}^{C} n_{c, k, l}=1-\epsilon
$$

Information needs to be transferred between the depletion solve on streamlines and the neutronics solve on a 2-D or 3-D mesh. The power and scalar fluxes are computed on the neutronics mesh and need to be available on the streamlines, while the isotope compositions are computed on streamlines and are required in order to compute the macroscopic cross sections on the neutronics mesh. The transfer from the neutronics model to the depletion model is denoted by $\mathbf{P}_{k}[\cdot]$ in Eq. 44. 
Currently, this transfer is performed through nearest node interpolation. This transfer does not guarantee that the total power of the reactor is conserved, meaning that we generally do not satisfy:

$$
\int_{\Omega} p(\vec{r}) d \vec{r}=\sum_{k=1}^{K} \int_{s_{k, \text { in }}}^{s_{k, \text { out }}} A_{k}(s) \mathbf{P}_{k}[p(\vec{r})] d s
$$

where $\Omega$ is the neutronics domain. To conserve the total power of the reactor, we correct the transferred power density and scalar fluxes by applying the scaling factor $\theta$ :

$$
\theta=\frac{\int_{\Omega} p(\vec{r}) d \vec{r}}{\sum_{k=1}^{K} \int_{s_{k, \text { in }}}^{s_{k, \text { out }}} A_{k}(s) \mathbf{P}_{k}[p(\vec{r})] d s}
$$

The isotopic compositions are transferred from the streamlines to the neutronics mesh using nearest node transfer as well.

\subsection{Numerical Results}

The results presented in this section have not been extensively checked and verified. Therefore, we caution the reader that all results are preliminary and subject to change; these changes will be reported in future reports or revisions of this report.

We consider a simplified radially symmetric pebble-bed reactor comprised of two regions: the fuel region and the radial reflector region. The upper and lower cone and axial reflectors are not modeled. Relevant specifications for this pebble-bed reactor are presented in Table 12. The specifications roughly correspond to a 250-MW, 6-pass design. For simplicity, the core is isothermal at $500 \mathrm{~K}$. Cross sections and transmutation/decay data are produced using the DRAGON [31] code. We condense the neutron cross section into a 9-group structure and use the 300 isotope transmutation/decay data library provided by DRAGON.

We solve the test problem using six burnup groups with boundaries at 7.48, 16.83, 22.44, 29.92, and 37.4 MWd/kg (initial heavy metal), and three streamlines located at $r=0.325,0.975$, and 1.4 $\mathrm{m}$ representing cylindrical shells located between radii $r=0$ and $0.65 \mathrm{~m}, r=0.65$ and $1.3 \mathrm{~m}$, and $r=1.3$ and $1.5 \mathrm{~m}$. We note that the discharge burnup of $37.4 \mathrm{MWd} / \mathrm{kg}$ is less than half what pebble bed reactors target. The discharge burnup follows from imposing the dimensions, 
Table 12: Specifications for the pebble-bed reactor test problem.

\begin{tabular}{ll}
\hline Parameter & Value \\
\hline Bed diameter & $3 \mathrm{~m}$ \\
Bed height & $10 \mathrm{~m}$ \\
Reflector thickness & $0.3 \mathrm{~m}$ \\
Rated power & $250 \mathrm{MW}$ \\
Average pebble residence time & $80 \mathrm{~d}$ \\
Discharge burnup & $37.4 \mathrm{MWd} / \mathrm{kg}$ (initial heavy metal) \\
Av. power density & $6 \mathrm{~W} / \mathrm{cm}^{3}$ (pebble volume) \\
Porosity (constant) & 0.4 \\
Tracked isotopes & 300 \\
Number of energy groups in neutronics solve & 9 \\
Neutronics solver & Griffin SAAF-P1 \\
Number of burnup groups & 6 \\
Number of different pebble types & 1 \\
Number of streamlines & 3 \\
Fresh pebble compositions & see Table 13 \\
\hline
\end{tabular}

residence times, and number of passes without iterating on these numbers to achieve a realistic discharge burnup. It does in no way invalidate the presented study because the specifics of the PBR design and its realism are inconsequential in this first demonstration of the equilibrium core depletion capability. The streamlines are axially discretized into 200 elements. The neutronics problem is solved in axisymmetric geometry using a orthogonal mesh with 24 elements in the radial direction and 200 in the axial direction. The model reactor is refueled from the bottom, and fresh pebble compositions are presented in Table 13.

Table 13: Tracked isotopes in the test problem.

\begin{tabular}{lc}
\hline Isotopes & Composition $[\mathrm{at} / \mathrm{b}-\mathrm{cm}]$ (pebble volume) \\
\hline U234 & $1.81 \mathrm{E}-07$ \\
U235 & $2.26 \mathrm{E}-05$ \\
U238 & $2.37 \mathrm{E}-04$ \\
O16 & $5.19 \mathrm{E}-04$ \\
O17 & $1.97 \mathrm{E}-07$ \\
B10 & $4.56 \mathrm{E}-08$ \\
B11 & $1.84 \mathrm{E}-07$ \\
Graphite & $1.42 \mathrm{E}-01$ \\
SI28 & $5.23 \mathrm{E}-04$ \\
SI29 & $2.66 \mathrm{E}-05$ \\
SI30 & $1.75 \mathrm{E}-05$ \\
C12 & $5.67 \mathrm{E}-04$ \\
\hline
\end{tabular}


The power density as well as the group 1 and 9 scalar flux distributions are plotted in Fig. 10. The power peak is shifted to the bed inlet, where the concentration of fissile isotopes is higher and the concentration of parasitic absorbers is lower. As a first check we ensure that the fast flux follows the power density closely. A second check is the presence of the thermal flux peak close to the reflector.
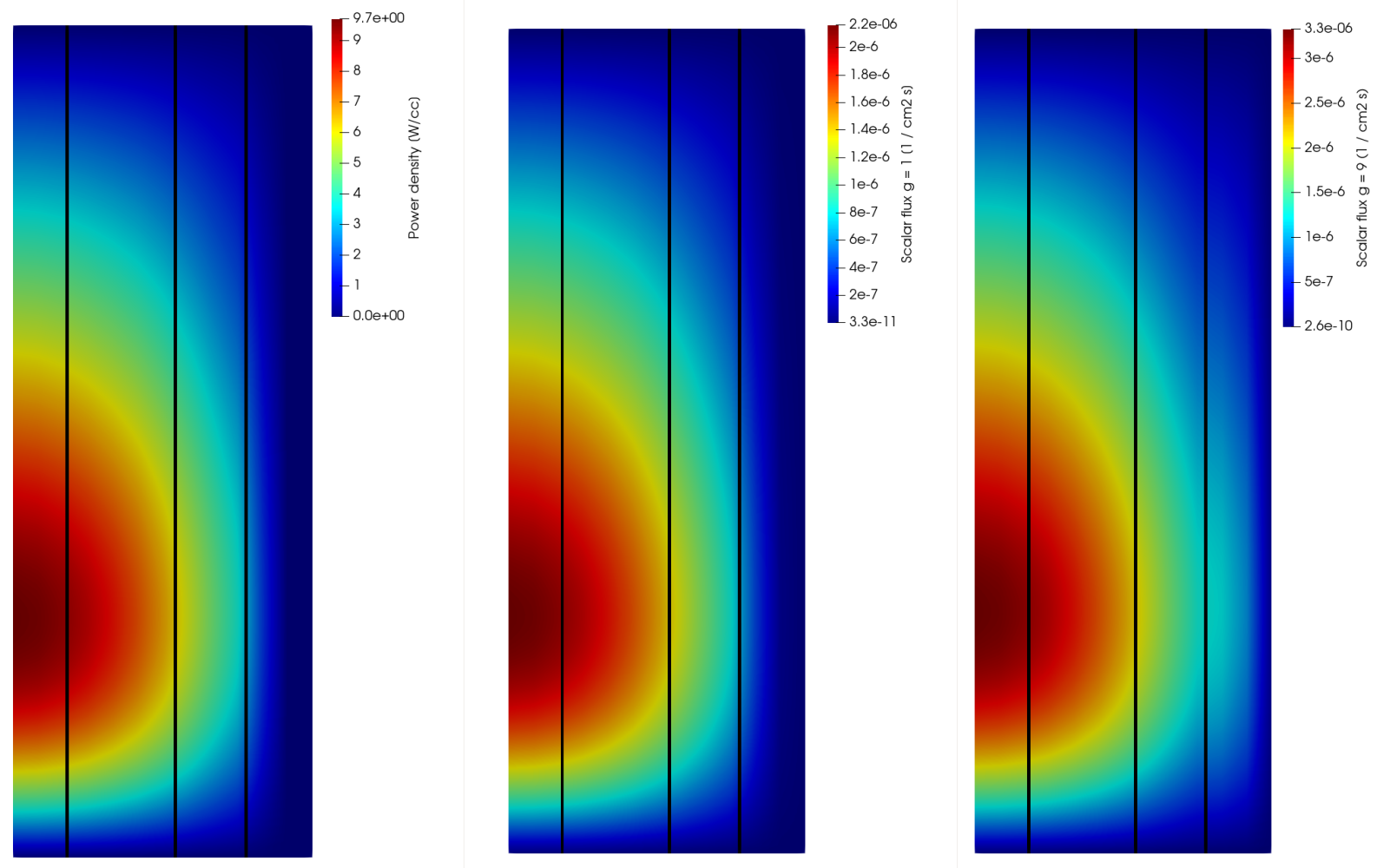

Figure 9: Power density (left), scalar flux of group $1\left(1.96 \times 10^{7}-1.95 \times 10^{5} \mathrm{eV}\right.$, middle), and scalar flux of group $9\left(1.38 \times 10^{-1}-1.10 \times 10^{-4} \mathrm{eV}\right.$, right). The scalar fluxes are not scaled to the power level of $250 \mathrm{MW}$. The three streamlines are overlaid as black lines, with streamline 1 near the core center and streamline 3 near the reflector.

The pebble volume fractions for burnup groups 1 through 6 on streamlines 1 through 3 are presented in Fig. 10. The most interesting burnup groups are the first, the last, and the second (upper left subplot in Fig. 10). The first burnup group does not have any source term within the core so its volume fraction can only decrease along the streamline. The rate of reduction is larger for larger power densities so the volume fraction of pebble group 1 drops quicker in channels 1 than in channels 2 or 3 . The volume fractions of burnup group 2 exhibit a maximum that tends to move to the inlet with increasing power density. The maximum is caused by competing source 
(from group 1) and sink (to group 3) terms. Volume fractions for the last burnup group can only increase and they increase faster for larger power densities. The intermediate burnup groups 3-5 show much less change from the inlet to the outlet as seen by the different scale of the y-axes. We verified that the pebble volume fractions of the burnup groups sum to the packing fraction (or equivalently 1 minus the porosity).
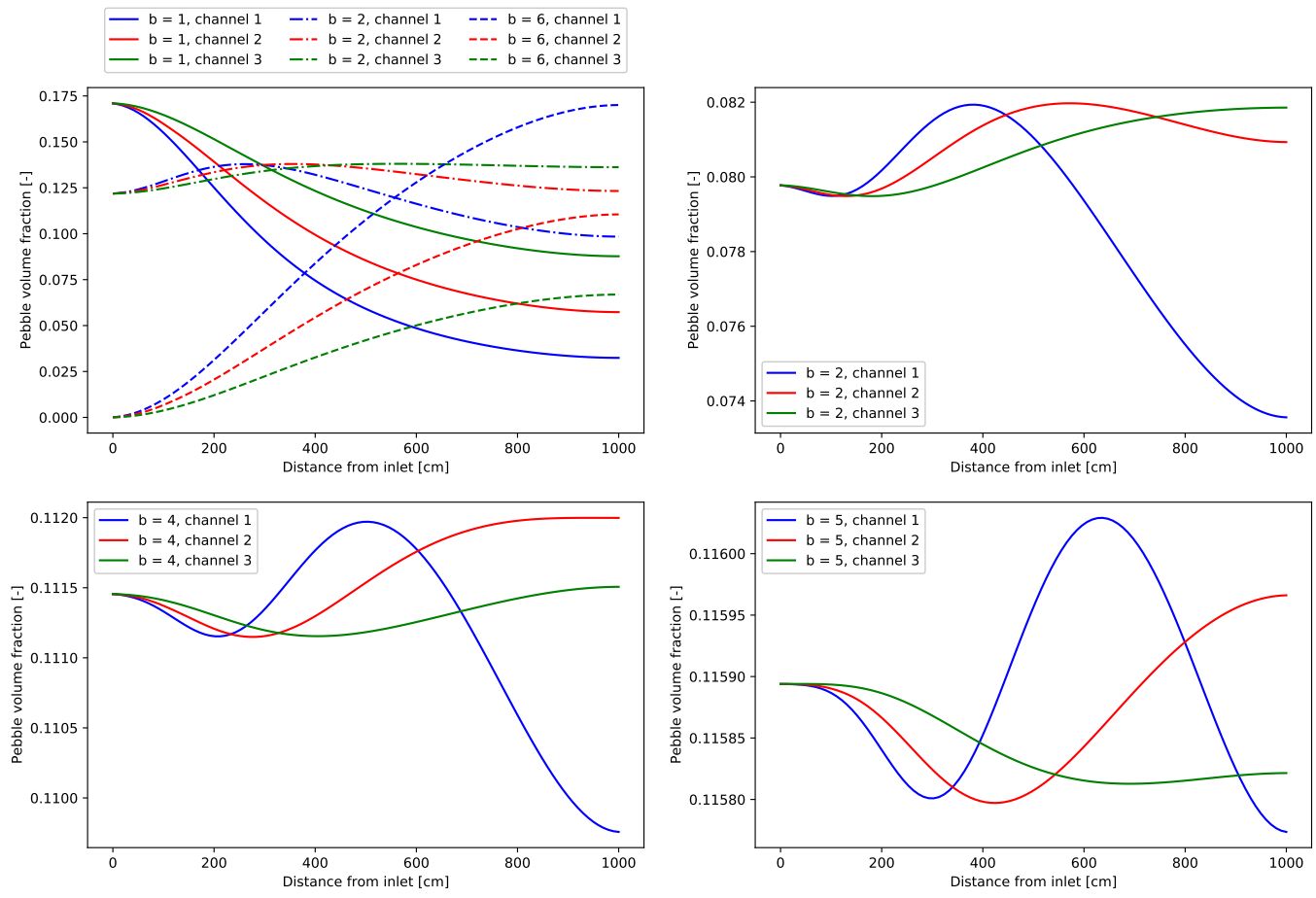

Figure 10: Pebble volume fractions for streamlines 1 through 3 and burnup groups 1 through 6 plotted versus distance from inlet.

Concentrations for selected isotopes are shown in Figs. 11, 13, XE-135 and I-135 concentrations (see Fig. 11) are large where the power density is large. Axially, I-135 peaks where the power density peaks; radially, the concentrations are largest in channel 1 and reduce towards the reflector. Equilibrium XE-135 and I-135 concentrations for power densities of 2, 5, 10, and $15 \mathrm{~W} / \mathrm{cm}^{3}$ (total volume) are listed in Table 14. The equilibrium concentrations are computed for an infinitely large system with an isotopic composition equal to the fresh pebble concentration (Table 13). The power density experienced by pebbles is expected to be somewhere in the range of $2-10 \mathrm{~W} / \mathrm{cm}^{3}$ while making their way through the inlet region of the core. The expected concentrations of I-135 and XE-135 agree well with the computed equilibrium concentrations. For I-135 and streamline 1 
at an axial location near the peak, we expect power densities close to $10 \mathrm{~W} / \mathrm{cm}^{3}$ and thus a concentration of I-135 of about 6 to $7 \times 10^{-10}$, which is close to the observed value. The computed XE-135 concentration for the equilibrium core is of the order of $1 \times 10^{-10}$ and increases with increasing power density. The increase from channel 3 to 1 is much smaller than the increase of I-135. The overall order of magnitude of XE-135 concentration and the observed trends agree well with the XE-135 equilibrium concentrations listed in Table 14 . We note that the increase of XE135 with power density is much smaller than the increase of I-135 with power density because I-135 concentration is directly proportional to the power density, while the concentration of XE135 asymptotes to a constant limit as the power density is increased. As we are quite close to this limit, the XE-135 concentration depends on power density in a much weaker manner than I-135.

The PU-239 and PU-240 concentrations increase from the reactor inlet to the reactor outlet. Radially, the concentrations of both PU-239 and PU-240 decrease from the inside of the bed to the outside (from channel 1 to channel 3). PU-239 has roughly a five times larger concentration than PU-240. At the outlet, the ratio of PU-239 to PU-240 is 6.84, 7.29, and 7.31 for channels 1 through 3 , respectively.

Finally, the U-235 concentration (Fig. 13) reduces from the inlet to the outlet (as expected, since it is consumed and hardly produced). The concentration also reduces more slowly in the channels that see less power density (channel 1 sees the fastest decrease, followed by channel 2, then channel 3).

Table 14: Equilibrium concentrations of XE-135 and I-135 for different power densities.

\begin{tabular}{lcc}
\hline & \multicolumn{2}{c}{ Concentration $[\mathrm{at} / \mathrm{b}-\mathrm{cm}]$ (total volume) } \\
\cline { 2 - 3 } Power density $\left[\mathrm{W} / \mathrm{cm}^{3}\right]$ (total volume) & XE-135 & $\mathrm{I}-135$ \\
\hline 2 & $9.02 \mathrm{E}-11$ & $1.36 \mathrm{E}-10$ \\
5 & $1.24 \mathrm{E}-10$ & $3.39 \mathrm{E}-10$ \\
10 & $1.42 \mathrm{E}-10$ & $6.78 \mathrm{E}-10$ \\
15 & $1.49 \mathrm{E}-10$ & $1.02 \mathrm{E}-09$ \\
\hline
\end{tabular}




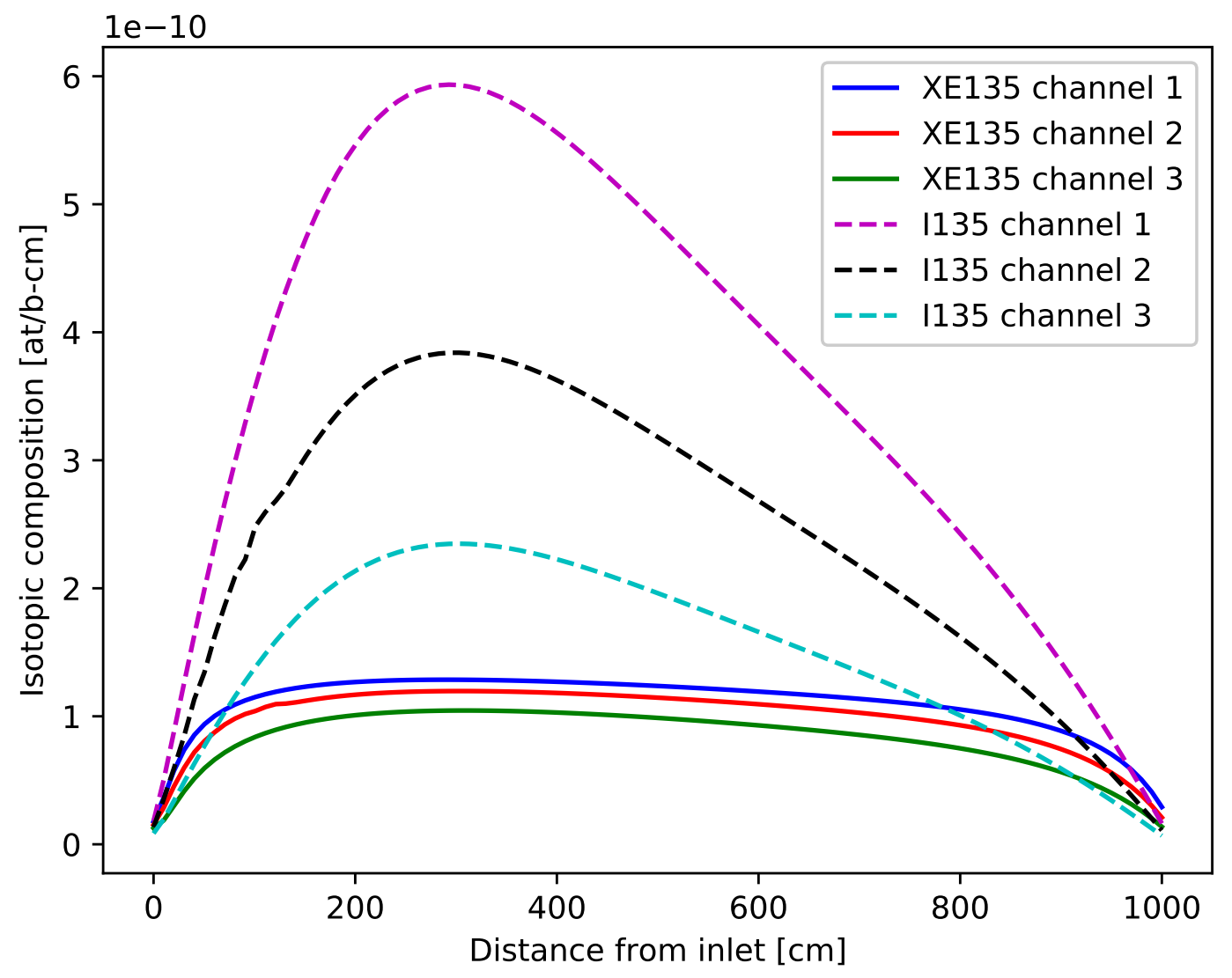

Figure 11: Concentration of XE-135 and I-135 (per total volume) plotted vs. distance from the inlet for channels 1-3. 


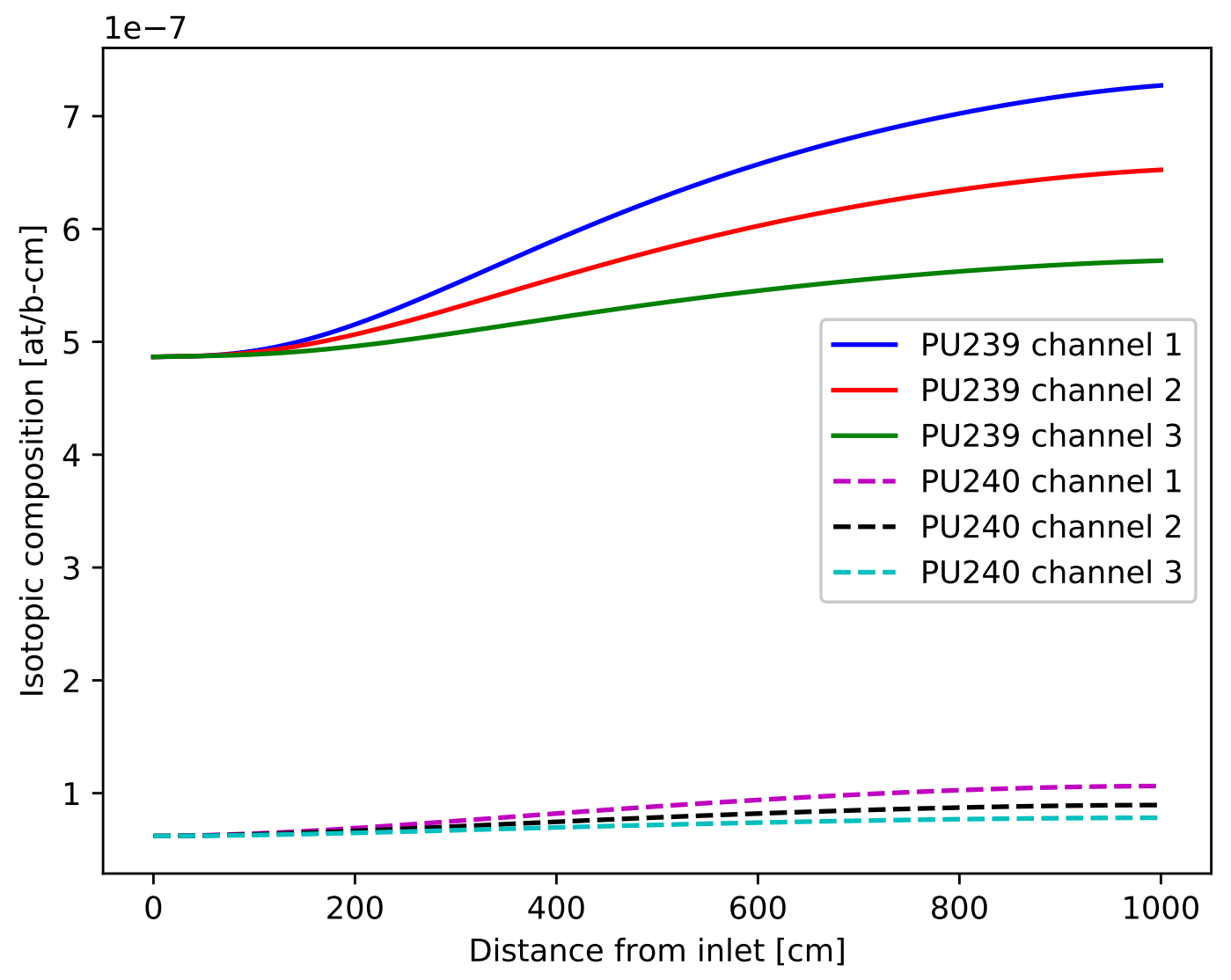

Figure 12: Concentration of PU-239 and PU240 (per total volume) plotted vs. distance from the inlet for channels 1-3. 


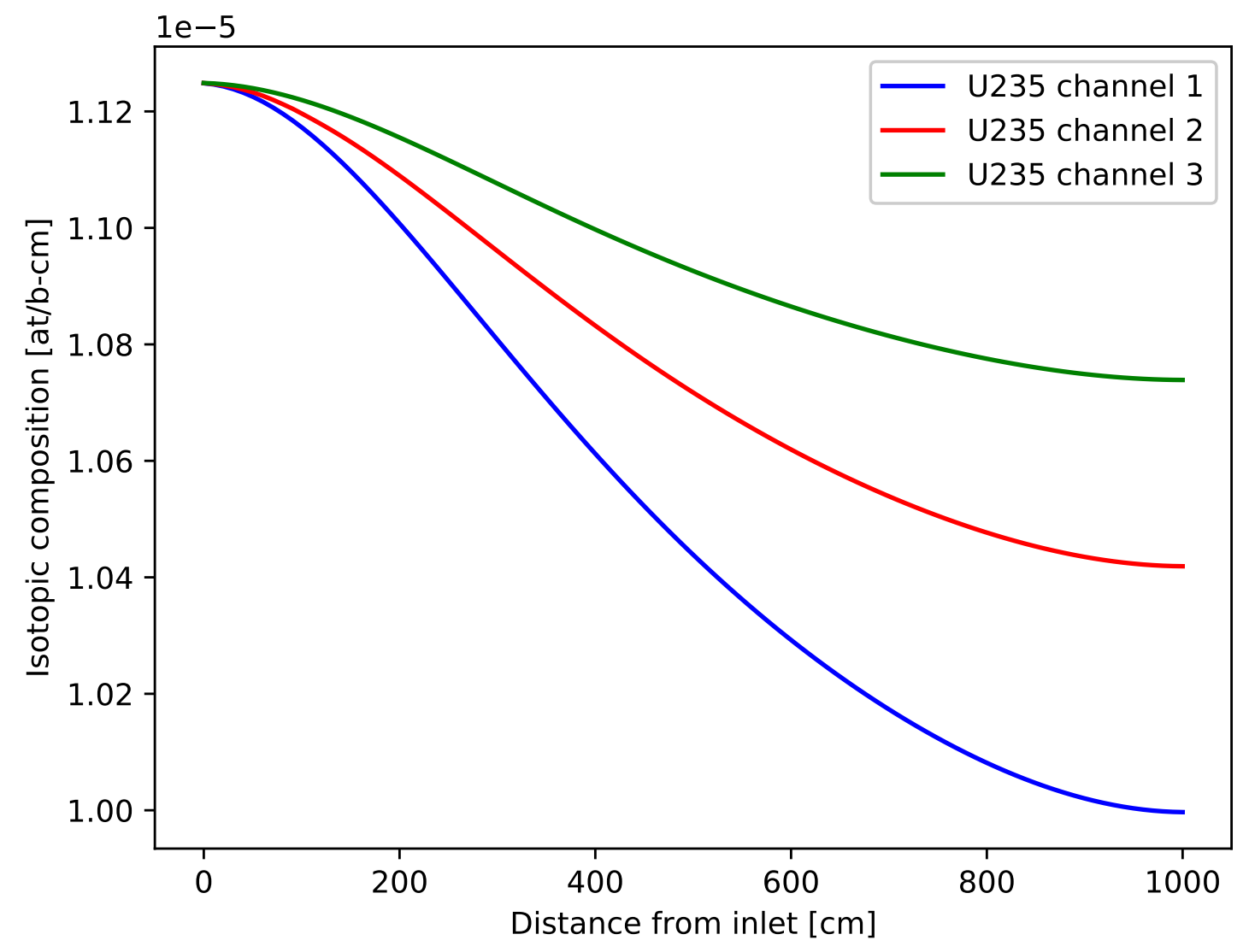

Figure 13: Concentration of U-235 (per total volume) plotted vs. distance from the inlet for channels $1-3$. 


\section{MOLTEN SALT PEBBLE-BED REFERENCE PLANT}

To demonstrate the modeling capabilities of MOOSE as a multiphysics reactor simulation tool, a Mark-1 Pebble-Bed Fluoride-Salt-Cooled Reactor (Mk1-FHR) model was built in MOOSE. The model uses Griffin and Pronghorn [9]-the neutronics and intermediate fidelity flow MOOSE applications, respectively-as well as the MOOSE heat conduction module. This suite of tools is aimed at providing medium-fidelity modeling of the plant-accurate enough that complex behaviors such as accident response or fluid-structure interactions may be studied, but simple enough that simulations can be executed on a desktop computer within a reasonable runtime, usually of the order of minutes to a few hours.

We will first describe the plant along with features of the model in Subsection 4.1. Next, we describe the neutronics model in Subsection 4.2, the thermal hydraulics model in Subsection 4.3 . the fuel performance model in Subsection 4.4, the primary loop model in Subsection 4.5, and finally the coupling of all these individual components in Subsection 4.6. When available, numerical results accompany the model descriptions and if possible are validated against higher-fidelity methods. Finally, future modeling efforts are described in Subsection 4.7 .

\subsection{Plant Description}

The Mk1-FHR — designed primarily at the University of California, Berkeley, in collaboration with other universities and national laboratories-is a Gen-IV-type reactor. It is described in great detail in [32] and is the topic of numerous $\mathrm{PhD}$ dissertations [33-36]. The primary loop coolant, a Fluoride-Lithium-Beryllium salt (FLiBe), enables it to reach higher temperatures than the current light water reactor (LWR) fleet and many competing Gen-IV concepts, making it economically attractive for industrial heat applications or hydrogen gas production [37, 38]. The fuel, described in more detail in Subsection 4.1.2, remains stable and retains fission products for temperatures above $1500^{\circ} \mathrm{C}$ making it naturally more resistant to loss-of-coolant transients. We will evaluate this resistance through transient modeling of the plant.

The rated thermal power output of the Mk1-FHR is $236 \mathrm{MWth}$, and a plant could include up to 12 modules of this reactor type. Modular construction is expected to reduce costs through increased offsite assembly, reduce construction difficulties (unlike with large single-core units), 


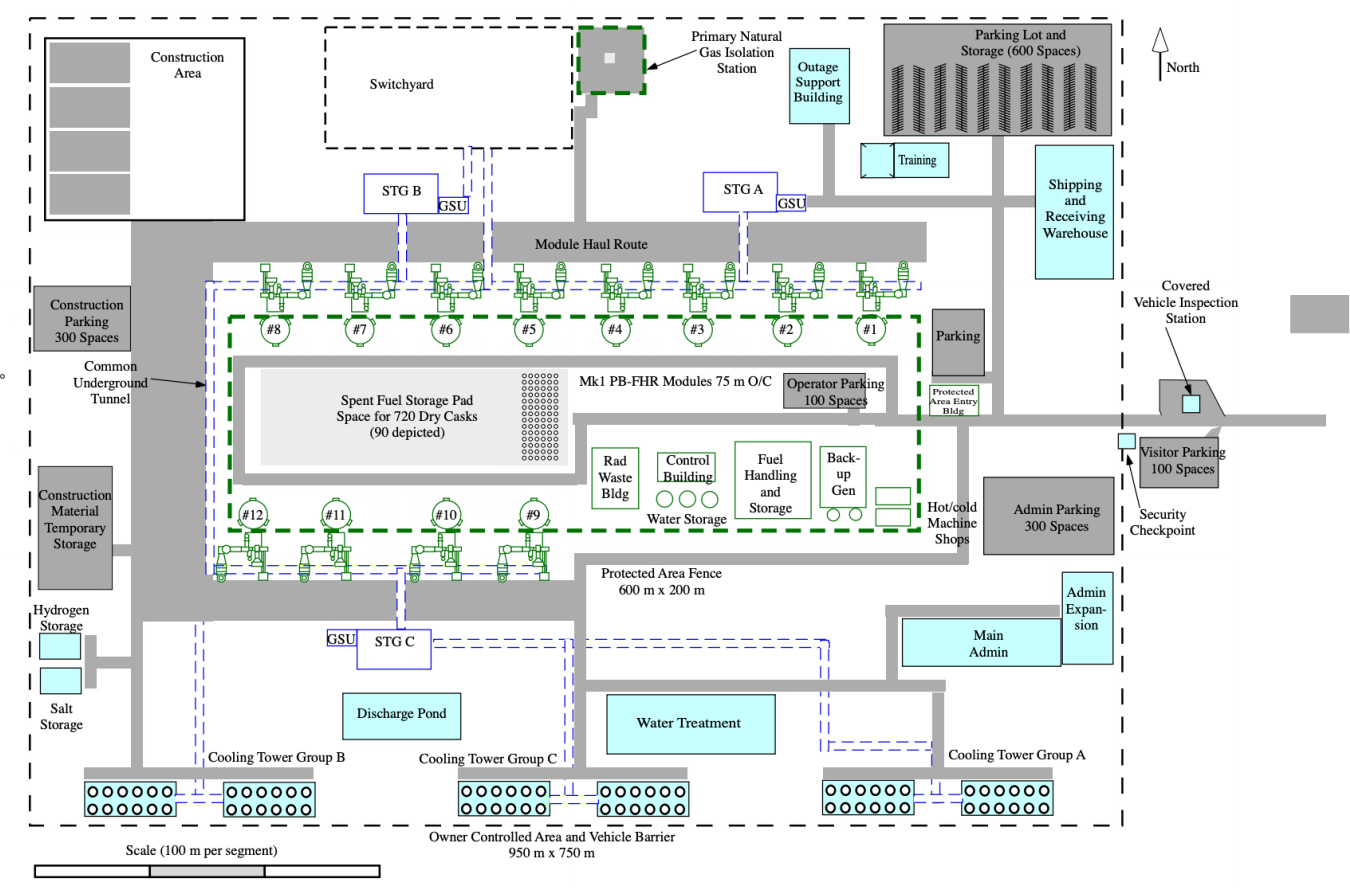

Figure 14: Mk1 site arrangement for a 12-unit, 180-acre PB-FHR plant capable of producing a 1200 MWe base load and 2900 MWe peak, from [32].

and decrease financial risks via lower upfront capital costs for the initial unit. The full plant layout is represented in Fig. 14, and a single unit and its heat extraction system is represented in Fig. 15.

\subsubsection{Core}

The core of the Mk1-FHR is shown in Fig. 16 inside the reactor vessel. It is composed of an inner reflector, a fuel pebble bed, a graphite reflector pebble bed, and an outer reflector. These core components are enclosed in the core barrel. The salt downcomer is located on the outside of the core barrel. Thermal insulation is provided by a thick brick wall. We assume air cooling on the outside of the brick wall. Both pebble regions were assumed to have a pebble packing fraction of 0.6. Molten salt flows from the bottom to the top of the core, but also enters through channels from the inner reflector and exits from a bypass plenum outlet and through tubes in the outer reflector. Pebbles are fed through the bottom inlet of the core and defueled in the defueling chute at the top of the core. Pebbles perform multiple passes through the core, as described in Sect. 3.2.5. The lower power density of the core is a design characteristic to ensure passive safety during accident scenarios. 


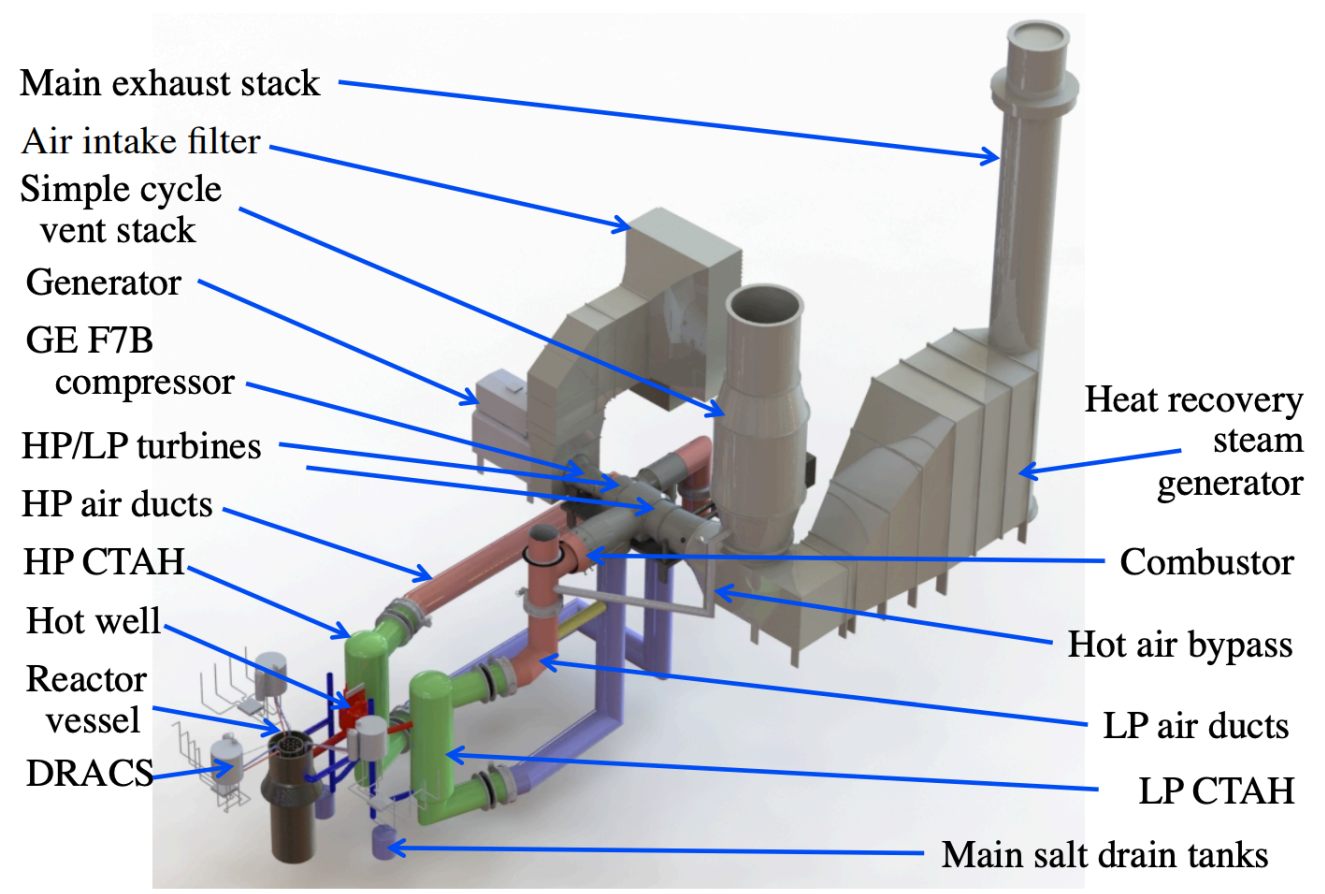

Figure 15: The Mk1 PB-FHR interface with its NACC power conversion system, from [32].

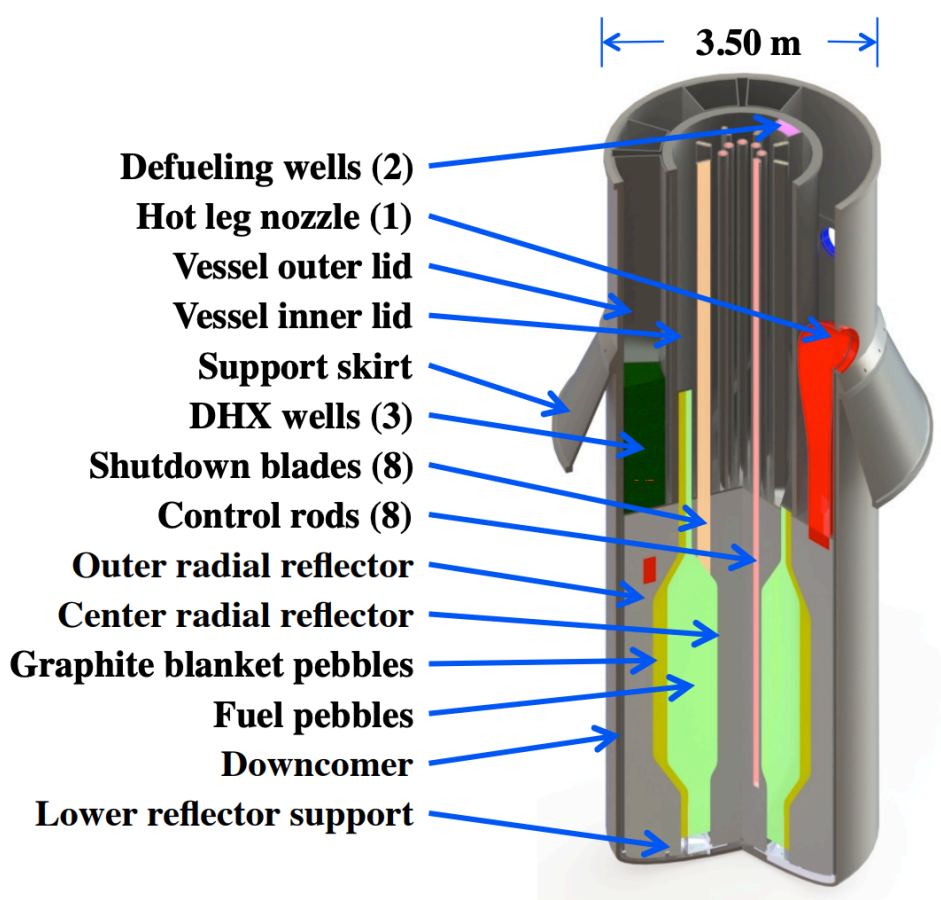

Figure 16: The Mk1 PB-FHR reactor vessel, from [32]. 


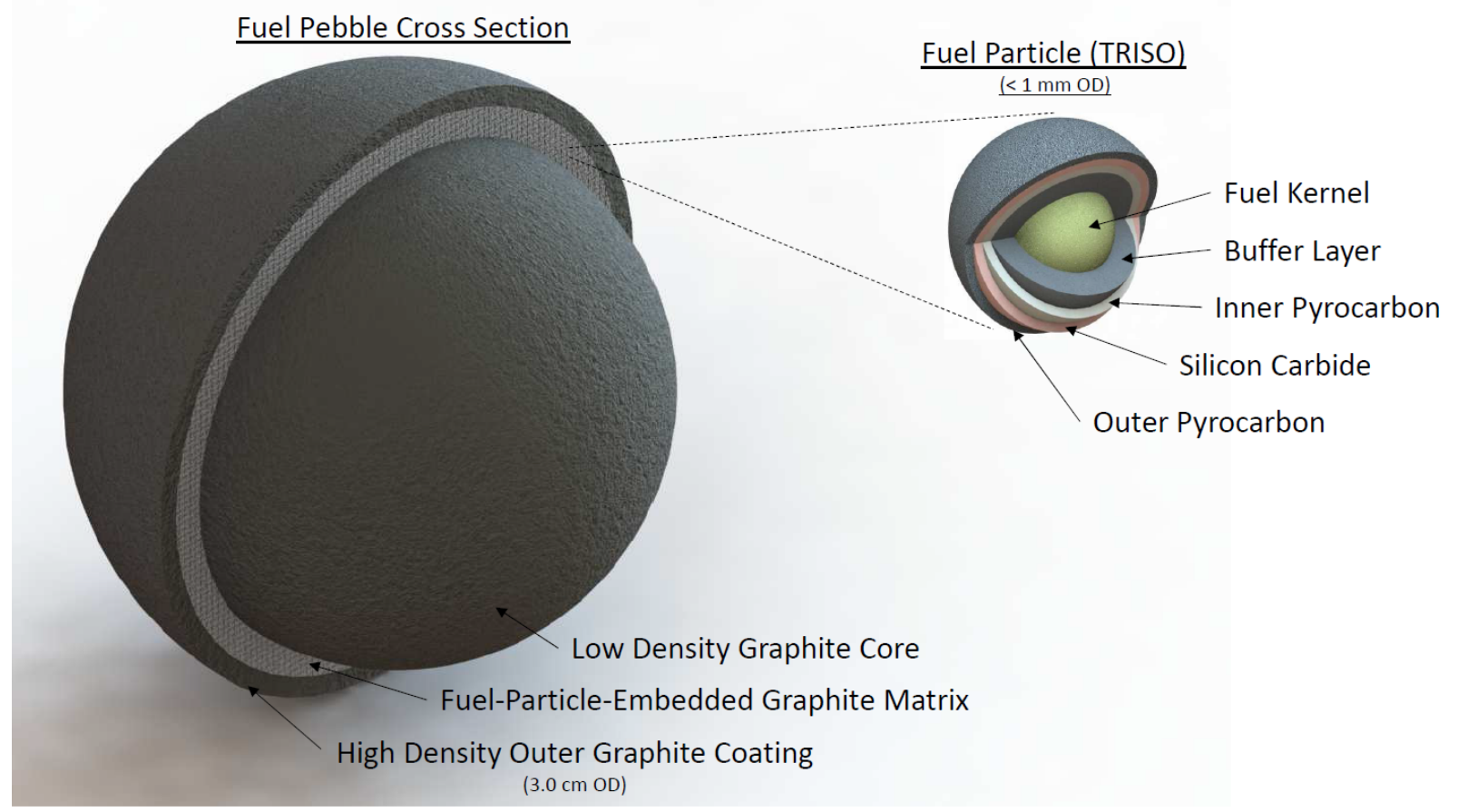

Figure 17: Fuel pebble and TRISO particle diagram, from [32].

\subsubsection{Fuel}

The fuel pebbles, as shown in Figure 17, are multi-layered fuel-graphite composites. The graphite outer shell and inner core enclose a fuel matrix made of TRi-structural ISOtropic (TRISO) fuel particles inside a graphite matrix. The inner core of each pebble provides buoyancy in the molten salt. The TRISO particles have a nearly $20 \%$-enriched oxide fuel core covered by layers of pyrolitic graphite and silicon carbide. This encapsulated fuel form is very stable during high-temperature transients, due to the TRISO's excellent thermal stability and its ability to retain fission gases.

\subsection{Neutronics Model}

\subsubsection{Cross-Section Generation}

PB-FHR neutronics still hold numerous challenges [39], and the generation of group cross sections for these reactors is a rather complicated endeavour, having to account for the multi-scale doubleheterogeneity effect [40]. Direct homogenization of the pebbles and TRISO particles is highly inaccurate [41], and deterministic methods based on collision probabilities [42] or transport theory for stochastic media [43] were developed to treat these effects. 
To avoid these challenges, a Monte Carlo simulation was used for neutron group cross-section generation. This choice is increasingly common in the nuclear reactor physics community, with Monte-Carlo-generated group cross sections having been used for studies conducted on small modular reactors [44], VVERs [45], PWRs [46, 47], and advanced reactors such as a molten salt fast reactor [48], a sodium fast reactor [49], a super-critical water reactor [50], and a lead-cooled reactor [51]. The main advantage of this approach is that it uses both a fully heterogeneous exact representation of the reactor, avoiding both geometrical approximations and/or initial homogenization approximations, and continuous energy representations of group cross sections, avoiding the need for complex resonance models for intra- and inter-nuclide resonance interference. We also use full-core calculations instead of lattice/infinite medium calculations for group crosssection generation, obviating the need for leakage-correction models and naturally modeling environmental effects such as proximity to the reflector.

Serpent 2 [52] is used for group cross-section generation. Its use of Woodcock delta-tracking [53] reduces the computational burden of computing numerous surface intersections in a pebble-bed reactor, with TRISO particles modeled explicitly. Serpent 2 computes accurate neutron diffusion coefficients via the B1-inscatter method [54]. Serpent 2 has been used for group cross-section generation, mostly in the context of nodal diffusion calculations [44, 49, 52]. Thermal scattering data will be used for graphite but not the salt, even though it was identified as an important physical phenomenon to model [55], as the data is not currently readily available. The input files are generated using the input file generation from [33], and the same 8-group energy structure is used. The entire core is currently filled with pebbles; further studies will adjust the fuel content.

To cover the entire range of the transient conditions, group cross sections and delayed neutron data are generated for a wide range of operating conditions, as summarized in Table 15. The coolant density is adjusted by temperature, using the linear dependence shown in Equation 68 . For the purpose of group cross-section generation, the fuel and coolant temperatures are assumed uniform in the entire core. Further study may consider multi-region models. The continuous energy cross sections are evaluated at the selected temperature points. A sensitivity study will also be conducted to examine the adequacy of the chosen number of steps per parameter.

$$
\rho_{\text {flibe }}\left(\mathrm{kg} / \mathrm{m}^{3}\right)=2413-0.4884 \cdot T_{\text {flibe }}(K)
$$


The group cross sections are tallied on a cylindrical mesh corresponding to the Griffin 2D-RZ model described in Subsection 4.2.2

Table 15: Parameter grid for Monte Carlo group cross-section generation.

\begin{tabular}{ccc}
\hline Parameter & Range & Steps \\
\hline Pebble temperature & {$[300,1500 \mathrm{~K}]$} & 5 \\
Salt temperature & {$[300,1500 \mathrm{~K}]$} & 5 \\
Reflector temperature & {$[300,1500 \mathrm{~K}]$} & 5 \\
Control rod insertion & {$[0,100 \%]$} & 5 \\
\hline
\end{tabular}

One refinement upon the model built by [33] is the introduction of random pebble packing. Ordered packing was shown to be a major approximation in [55]. Figs. 18a and 18b show a radial cut of the model with ordered and random pebble packing. The random packing was obtained using the Serpent 2 disperse utility, ran for over 24 hours to generate a position file of over 320,000 pebbles in the 3-D core. The random pebble distribution was only generated for the central part of the core, as the utility does not currently support cones and a fortiori cone intersections. Future work may consider random TRISO packing in the pebble fuel matrix. The performance impact of introducing the random pebble distribution was minimal.

The Serpent 2 simulations have a reported uncertainty of $20 \mathrm{pcm}$ on the eigenvalue. Convergence of group cross sections is not uniform across all materials, but the overall reported uncertainty was below $0.1 \%$ for the most important materials (core, control rod, reflectors). This simulation consisted of 500 inactive batches followed by 2,000 active batches of 5,000 histories. This is not an optimal run strategy, and further effort may be employed to reduce this runtime or increase the number of histories. The overall number of particles, 10 million, is generally considered to be on the lower end to obtain a resolved power distribution. The reported $k_{\text {eff }}$ for face-centered-cubic (FCC) ordered and random pebble packings, with the same target porosity, can be found in Table16. The difference in $\mathrm{k}_{e f f}$ is only slightly below $40 \mathrm{pcm}$, the influence of the pebble packing on the group cross sections and on the power distribution was not quantified.

Table 16: Serpent 2 eigenvalue calculation results for ordered and random pebble packing.

\begin{tabular}{ccc}
\hline Packing & $k_{\text {eff }}$ & Single calculation runtime (on macbook, min) \\
\hline FCC ordered & $1.30337( \pm 20 \mathrm{pcm})$ & 75 \\
Random & $1.30290( \pm 20 \mathrm{pcm})$ & 80 \\
\hline
\end{tabular}




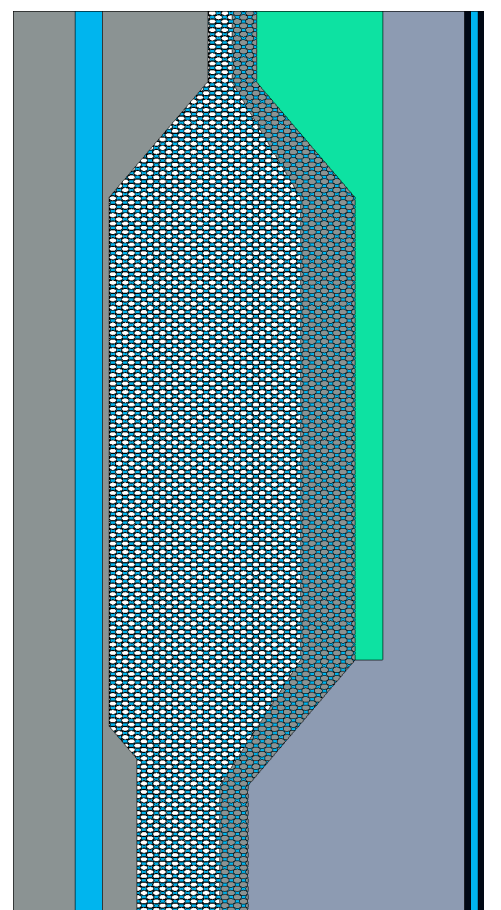

(a) Face-centered-cubic pebble packing

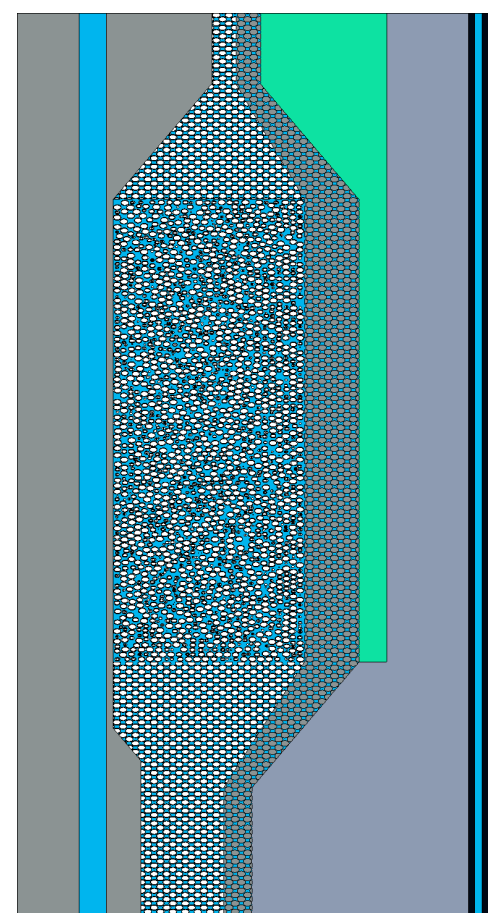

(b) Random pebble packing

Figure 18: Radial cut of the FHR core.

\subsubsection{Solution Method}

We use a 2-D RZ representation of the core in Griffin. The model domains are shown in Figs. 19a and $19 \mathrm{~b}$ for the case with a single and multiple fuel group cross-section regions, respectively. The model may be limited to all components inside the barrel, as very few neutrons ever stream past the barrel before reflection back inside the barrel.

The steady-state uncoupled eigenvalue 8-group diffusion equations were solved. We used the PJFNK non-linear eigenvalue solver to obtain convergence in four non-linear iterations. The coarsest mesh we used consisted of 1,626 triangular elements at an average size of $12 \mathrm{~cm}$. We refined this mesh uniformly across the entire model to study the convergence of the solution with reduction of mesh size.

Figs. 20 and 21 show the thermal/fast fluxes and power distributions computed for the PBFHR by Griffin, without thermal-hydraulics coupling. The thermal flux peak is due to the proximity of the reflector, which thermalizes neutrons while absorbing very few of them. The higherenergy neutrons making up the fast flux have a longer mean free path, and their distribution is driven by leakage. The power distribution is mostly driven by the thermal flux, as the fission cross 


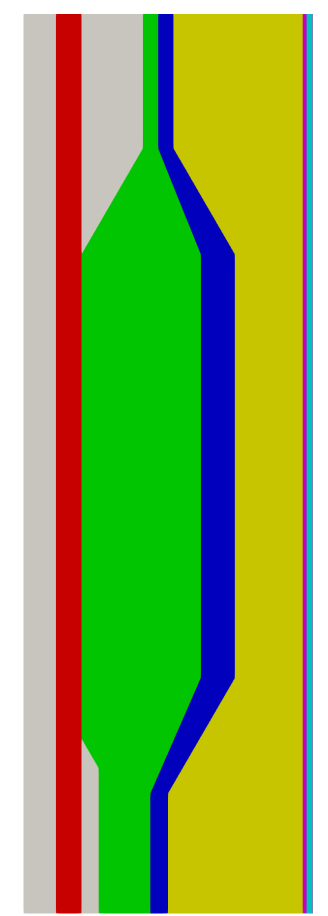

(a) Single fuel region

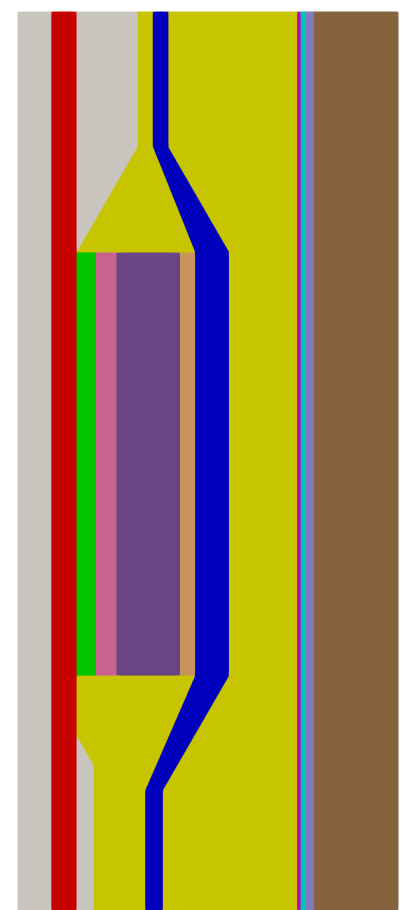

(b) Multiple fuel group cross section regions

Figure 19: Griffin PB-FHR model. This model does not include the plenum or the salt in the outer reflector.

sections are higher in the thermal range. While the power density is larger near the inner reflector, more power overall is generated in the outer parts of the core, due to their larger volume.

\subsubsection{Cross-code validation}

The solution obtained can be validated through comparison with the Serpent 2 power distribution and eigenvalue. In Serpent 2, we tallied the power distribution over a 20x20 grid laid out in a cylindrical uniform rectilinear mesh. Table 17 compares Griffin and Serpent 2 in regard to both physical quantities as well as various mesh refinements. Each level of refinement refines the mesh by a factor of four. We can see that the Griffin solution is converged to $100 \mathrm{pcm}$ for a four-fold uniform refinement, or a mesh size of $3 \mathrm{~cm}$. A perfect comparison is not expected, as there remains differences in the two mesh models; for example, the thin layer of reflector between the control rods channel and the core is not modeled in Griffin. The Serpent 2 model also includes salt in the outer reflector and plenum, which is homogenized throughout the outer reflector in Griffin. The power distribution is only compared in the central region, as the Serpent 2 tally mesh used 


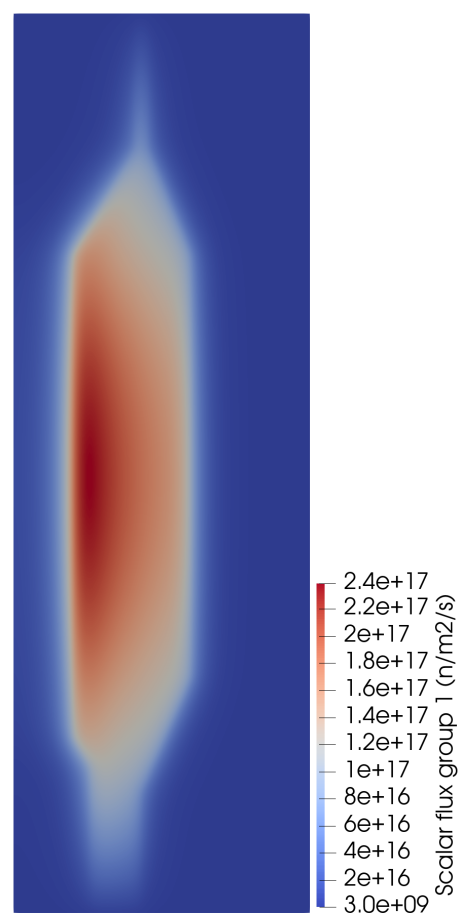

(a) Group 1 fast flux

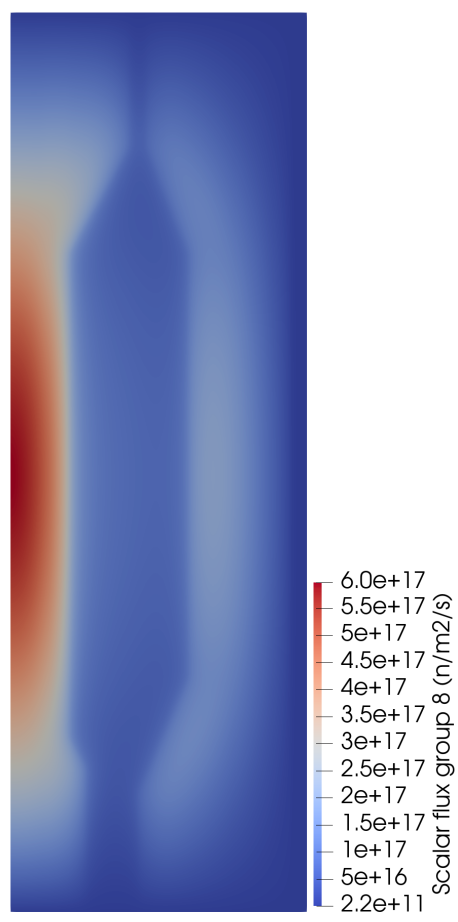

(b) Group 8 thermal flux

Figure 20: Neutron flux distributions generated by Griffin.

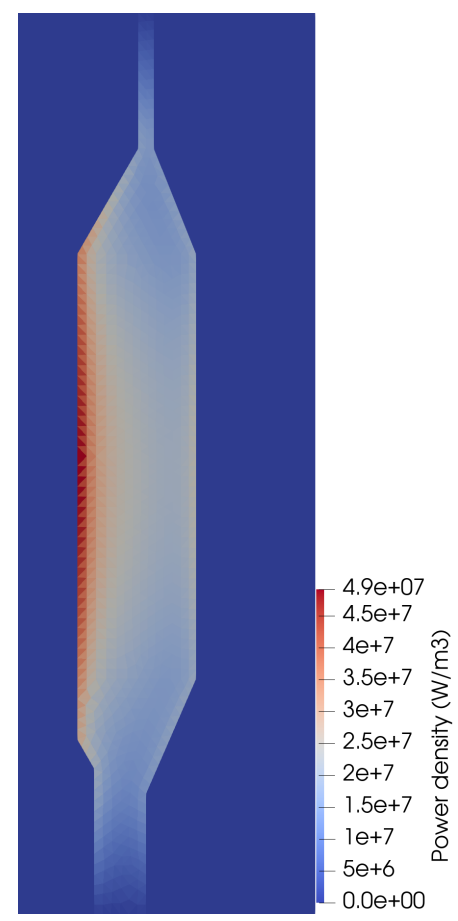

Figure 21: Power distribution generated by Griffin. 
is insufficient to resolve the expansion cones. Axially integrated or radially integrated comparisons between diffusion and Monte Carlo, presented in [41], are satisfactory and hide the errors at corners. Reporting the 3D distribution of error on a fine grid is a much more demanding metric.

Table 17: Griffin mesh convergence study and comparison to Serpent.

\begin{tabular}{cccc}
\hline Mesh uniform refinement $\left(4^{n}\right)$ & $\mathrm{K}_{\text {eff }}$ bias $(\mathrm{pcm})$ & max error $(\%)$ & RMS error (\%) \\
\hline $\mathrm{n}=0$ & 99 & 76 & 42 \\
1 & -75 & 60 & 39 \\
2 & -121 & 42 & 25 \\
3 & -133 & 38 & 22 \\
\hline
\end{tabular}

The comparison of the power distributions is shown in Fig. 22. The power distributions are only in strong disagreement on the top part of the central region, very close to the top cone. This is a region of lower power compared to the center of the core, where power is predicted more accurately. At the bottom, because of the offset between the two sides of the expansion cone, the plotted region is further away from the cone. We also expect these regions to be more sensitive to the model differences between Serpent 2 and Griffin mentioned in the previous paragraph, as well as more difficult to model with diffusion, given the increased heterogeneity.

The effect of this power distribution discrepancy on the fluid temperature distribution can be analyzed by using the reference source in the Pronghorn model. We did not pursue this analysis.

\subsection{Thermal-Hydraulics Model}

\subsubsection{Model Description}

A finite element model (FEM) of the porous media fluid flow was developed in Pronghorn based on an earlier model developed in preparation of Ref. [34]. We are currently developing a finite volume model (FVM) for the same equations; however, the results reported here use FEM, as the FVM is still under development. The motivations behind the development of the FVM are increased stability at a high Reynolds number, the ability to use a coarser mesh, and more consistent application of boundary conditions. The porous media fluid flow equations used in this work can be found in [56] and correspond to a friction-dominated model. The Pronghorn model geometry is shown in Fig. 23. The inlets on the inner reflector are modeled using a linearly varying mass 


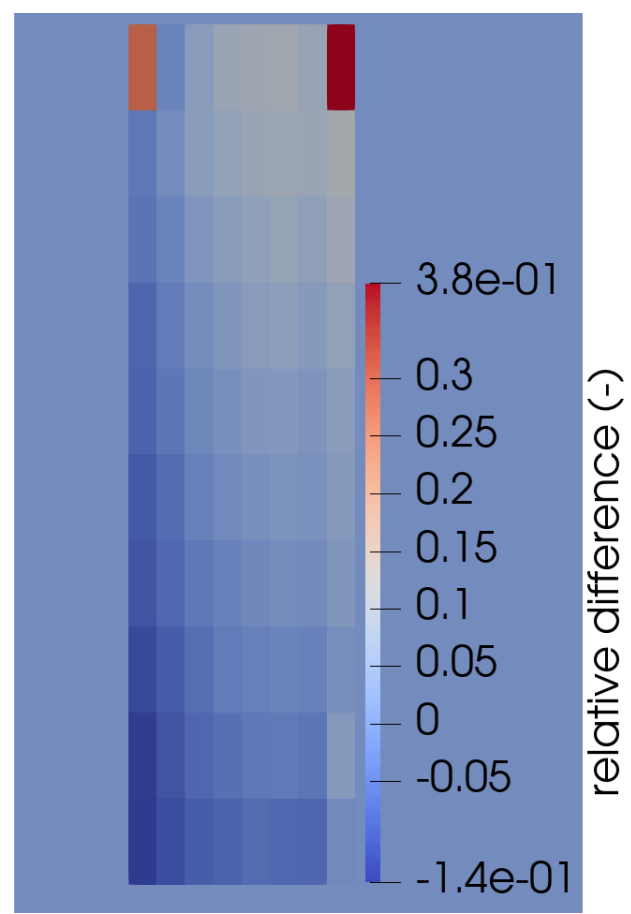

Figure 22: Relative difference between the Serpent 2 and Griffin (three levels of uniform mesh refinement) power distributions in the central part of the FHR core, tallied over a coarse mesh.

flow rate. The flow in the core plenum and outer reflector are modeled as well.

A full-core solid heat conduction model is coupled to the fluid flow model. The porous media equations for the solid temperature are defined in the pebble bed, and regular heat conduction occurs in the reflector and beyond the vessel and the brick wall.

For the stand-alone thermal-hydraulics model, we assume a cosine axial power source in lieu of the reactor physics output and solve for the fluid and solid temperature, as well as the pressure field. The results are shown in Fig. 24. The hotter salt exits the core mostly through the plenum, with a bypass fraction of nearly $90 \%$. This is because the friction predicted by the plenum isotropic drag model is lower than for the defueling outlet. Further studies may modify these friction coefficients. These may essentially be tuned to obtain the right flow fraction. The pressure drop (i.e., pressure difference of inlet and outlet) is close to 1 atmosphere and hence mostly due to the hydrostatic pressure loss. Further investigation into the drag models is required because the magnitude of the irreversible pressure drop seems unrealistically small. The solid temperature presents a strong gradient on the outer brick boundary, as a $100{ }^{\circ} \mathrm{C}$ fixed temperature boundary condition is applied there. The bricks have a much smaller thermal conductivity than the graphite in the 


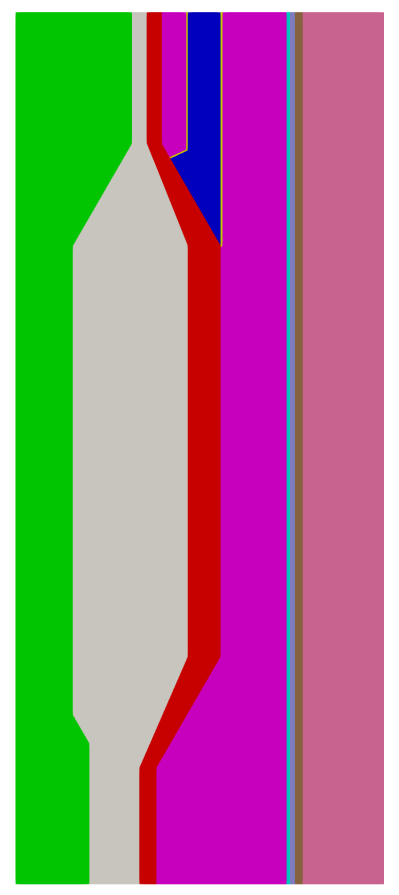

Figure 23: Pronghorn simulation domains. The plenum can be seen on top of the core as another outlet. The outer reflector is also a flow region.

reflectors leading to large thermal gradient in the bricks. In the future, this boundary condition may be modified to either convective and/or radiative boundary conditions.

\subsubsection{Verification \& Validation}

Verification of the thermal hydraulic model, once fully implemented in the finite volume formalism, will be performed by simply comparing it to the FEM. While the stabilization used at a high Reynolds number does influence results and will impede a FVM-FEM comparison, the FEM simulation should still provide an insightful comparison. We may also artificially reduce the Reynolds number and conduct the comparison in a flow regime in which tight agreement can be obtained between the FVM and non-stabilized FEM. The two implementations can be considered independent of each other, as they make use of different kernels, different boundary conditions, and were implemented by different developers.

Further verification of the thermal hydraulic model will be executed via direct comparison to a NEK-5000 computational fluid dynamics simulation being constructed at Argonne National Laboratory (ANL). Variables of interest will be the pressure drop and temperature distribution. 


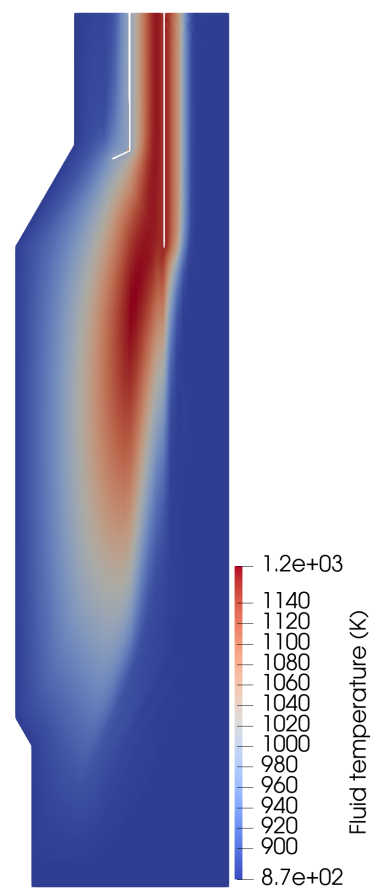

(a) Fluid temperature

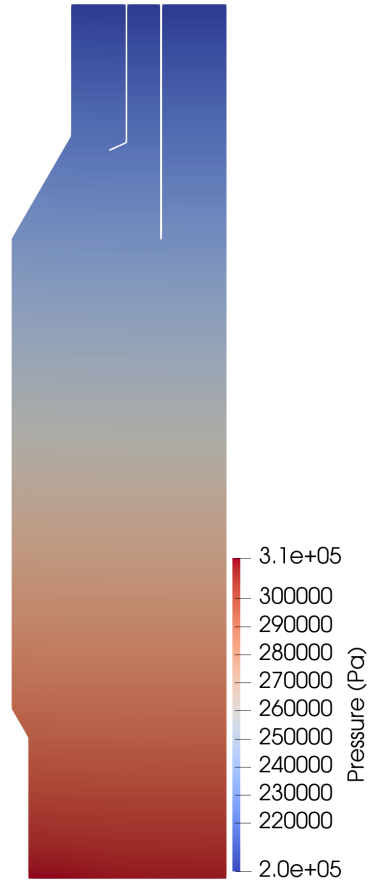

(c) Pressure distribution

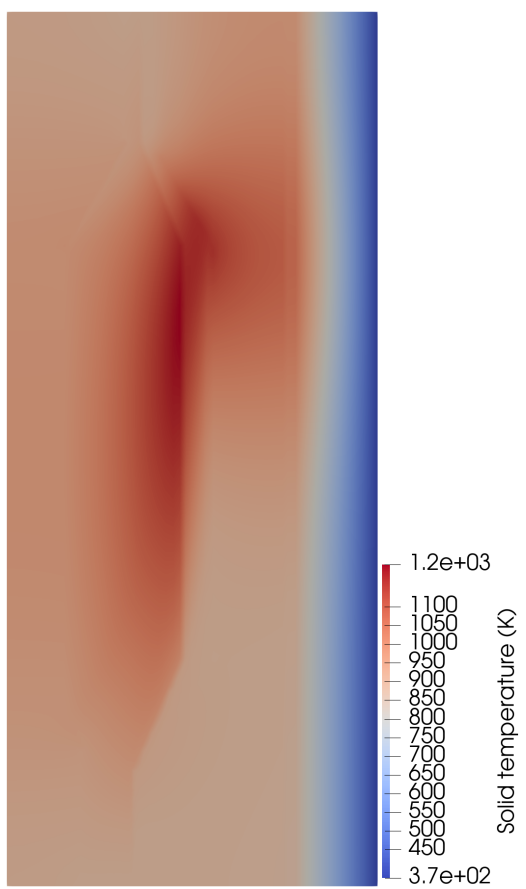

(b) Solid temperature

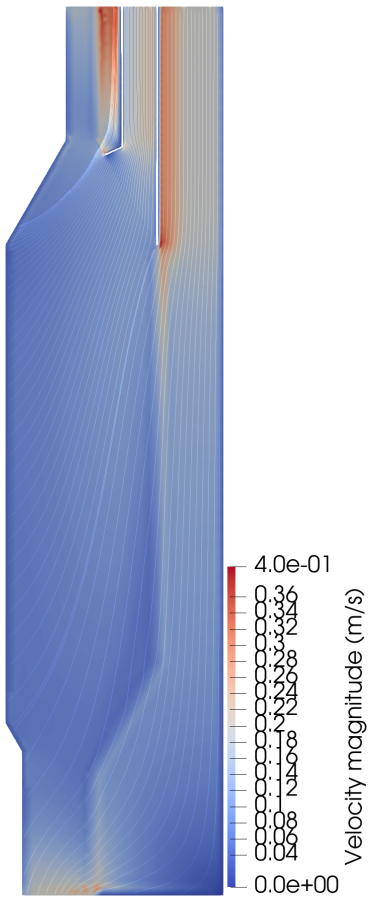

(d) Velocity magnitude and streamlines (measured from $\mathrm{z}=4 \mathrm{~m}$ )

Figure 24: Fluid temperature, solid temperature, pressure and velocity distributions obtained with the steady-state, stand-alone thermal-hydraulics Pronghorn model. 
The bypass flow will likely need to be adjusted in the Pronghorn model, based on the NEK-5000 results. Other closures, such as the effective thermal conductivity of the mesoscale solid medium, may also need to be improved through comparisons to high fidelity simulations.

Validation efforts were lead for Pronghorn based on the SANA experiments, which are gascooled pebble bed experiments. The results are detailed in [34]. Further validation was obtained by solving the PBMR-400 benchmark [57]. Future Pronghorn validation, as part of the NEAMS program, will leverage existing experimental facilities with the US laboratory and university system.

\subsection{Fuel Performance Model}

\subsubsection{Model Description}

The fuel temperature is obtained using a multiscale approach described in great detail and validated against high-fidelity models in [56]. We summarize the approach here and in Fig. 25.

When solved, the porous media equations provide the solid temperature. This solid temperature is used as the surface temperature of a 1-D pebble model. This 1-D pebble model can be solved on every element of the mesh, using MOOSE Multiapps. The porous media fuel temperature is then the outer Dirichlet boundary condition to the 1-D pebble model. The center boundary condition is naturally zero-gradient by symmetry. The fuel matrix temperature in this pebble model is itself used as an outer Dirichlet boundary condition in a 1-D TRISO+graphite matrix spherical model. Additional subtleties involve solving for only a correction to the temperature in this latter model. A similar approach is used for graphite pebbles, using only a pebble heat conduction model. A coarse mesh reduction is used to limit the computational cost of this approach.

\subsubsection{Avenues for Future Work}

The 1-D heat conduction model should eventually be replaced by a 1-D BISON model of the pebble, which can account for depletion effects on the thermal conductivity, provide extensive fuel performance information such as fission gas release rates, and model the pebble thermal expansion. 3-D spherical BISON models could be used to examine asymmetric conditions around the pebble, for example because of pebble-pebble contact or different flow conditions around the peb- 


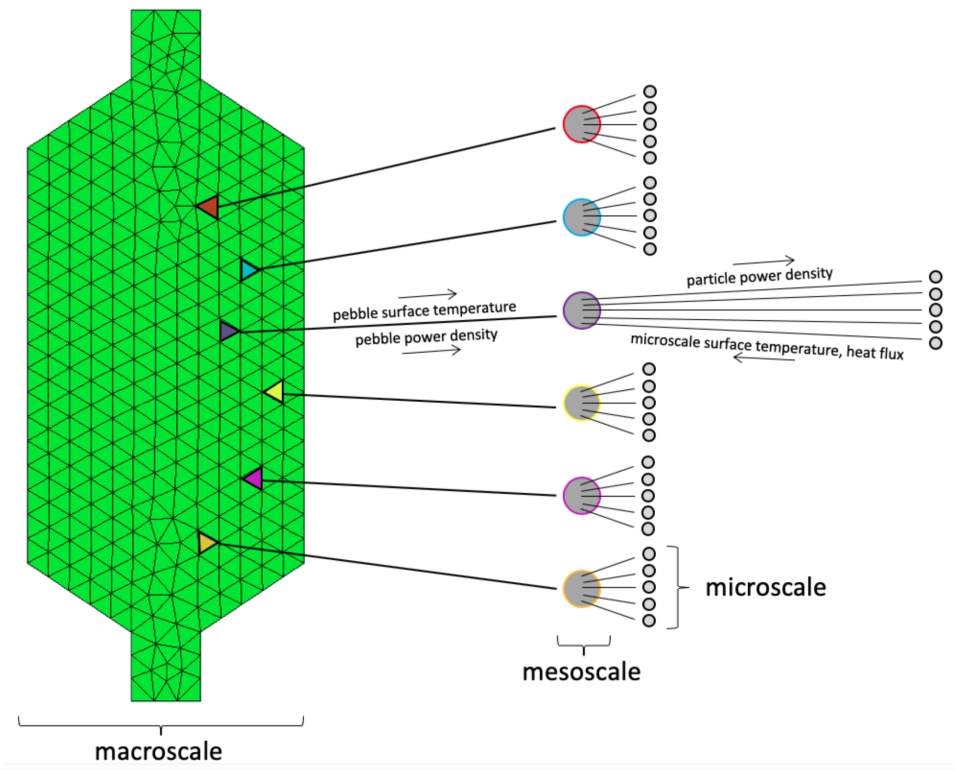

Figure 25: Multiphysics coupling between the thermal hydraulics model and the 1D fuel performance. A fuel performance application is spawned for each element. From [34]

ble. For the scope of this work, a temperature penalty factor or offset extracted from such calculations is likely

\subsection{Primary Loop Model}

Work is currently ongoing at Argonne National Laboratory (ANL) to develop a primary loop model of the Kairos-FHR in SAM [8] and to couple this model to the Pronghorn core model. SAM is a modern MOOSE-based system code designed for advanced reactors. The SAM system model will include a RCCS model that is required for accident simulations.

\subsection{Multiphysics Coupling}

\subsubsection{Steady-State Simulations}

The MOOSE framework enables easy coupling of the thermal hydraulics (Pronghorn) and the neutronics (Griffin) models described previously, as well as a heat-conduction-based model for the solids (fuel, reflector, etc.). Griffin provides the power density distribution, which is fed to both Pronghorn and the heat conduction model. The latter two converge pebble, salt, and reflector temperatures, which are then fed back to Griffin. Griffin uses this information to update the 


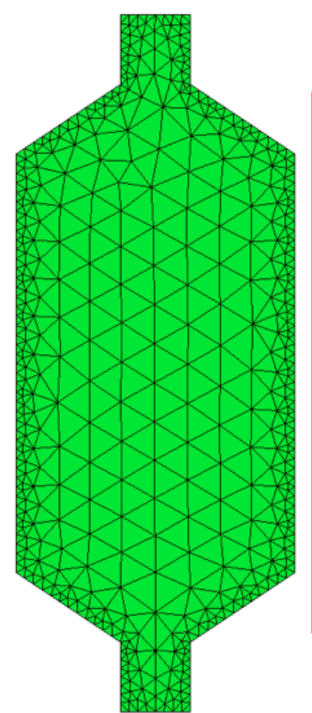

master application
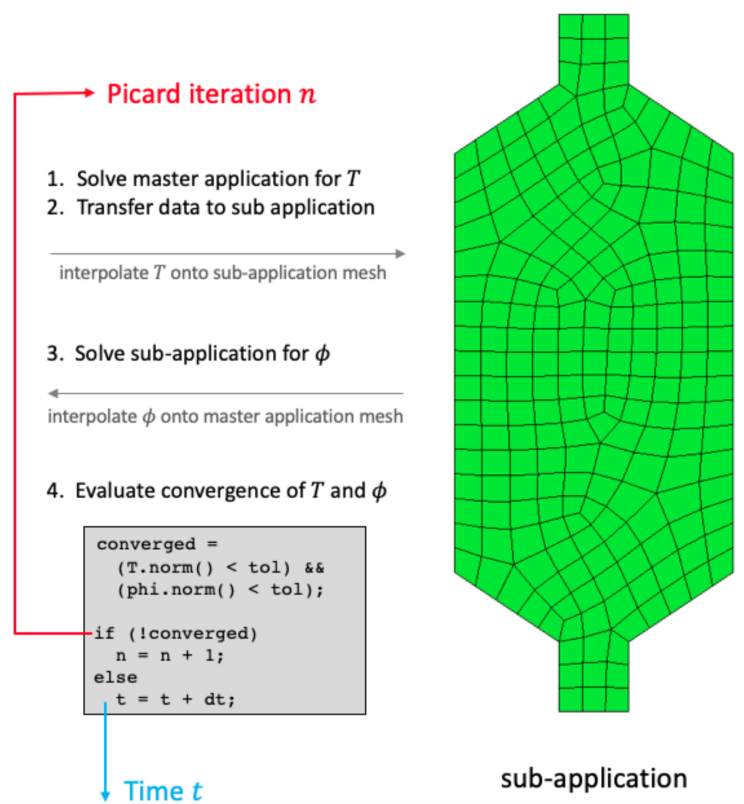

Figure 26: Multiphysics coupling between the thermal hydraulics model and the neutronics model (fuel performance omitted for clarity). Each physics is solved on a specialized mesh, limiting computational expense. Physical quantities such as the power distribution or the temperature fields are transferred between the applications. From [34]

group cross sections and re-generate a power distribution. This process is iterated to convergence in what is called "Picard iterations". The physical quantities are transferred over the different meshes using an interpolative and conservative transfer mechanism. Further development will consider the most physical way to transfer intensive physical variables such as temperature between models, likely by conserving other extensive physical quantities such as energy. The multiphysics coupling is illustrated in Fig. 26.

Fig. 27 shows the power density, neutron fluxes, solid and fluid temperatures, pressure, velocity field and $\mathrm{UO}_{2}$ temperatures for the converged multiphysics simulation, using all three models described previously. The power density peaks near the inner reflector in the core. Even though the fluid flow towards the bypass creates a temperature peak near the outer reflector, the stronger power generation near the inner reflector drives a higher fuel temperature there. The solid temperature drops sharply in the brick thermal insulation. Peak fuel temperature remains far below melting; further fuel performance studies may devise more stringent criteria. 


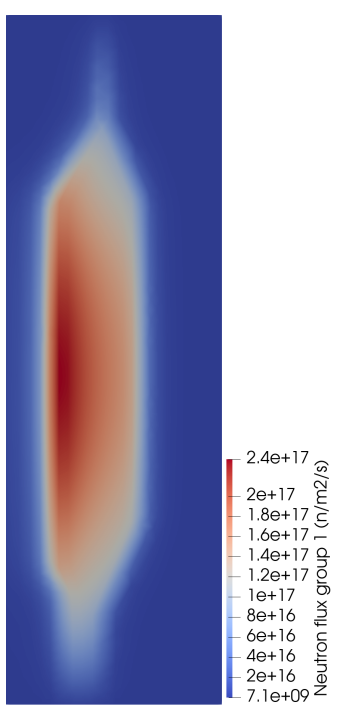

(a) Fast neutron flux (group 1)
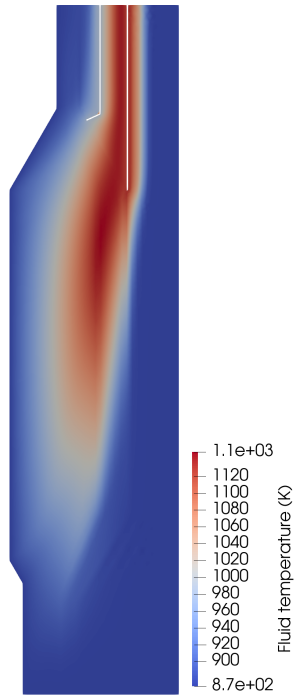

(d) Fluid temperature

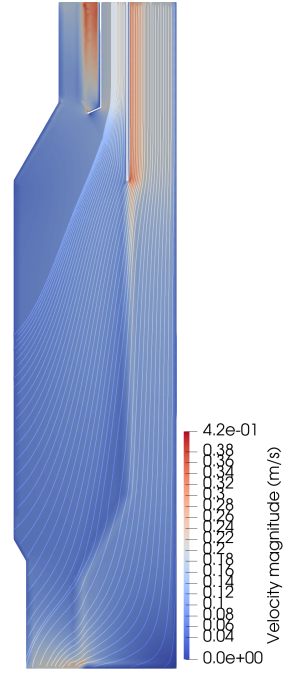

(g) Velocity streamlines

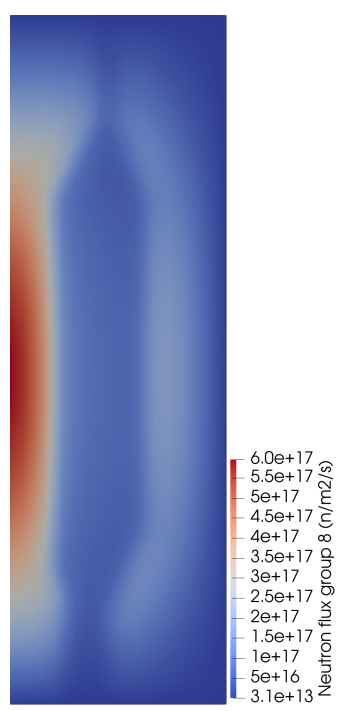

(b) Thermal neutron flux (gr. 8)

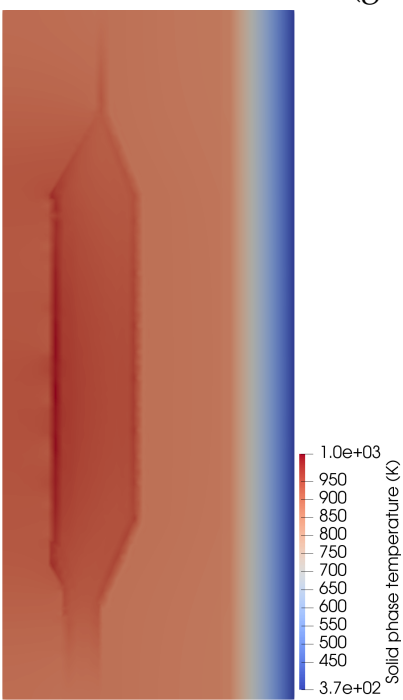

(e) Solid temperature

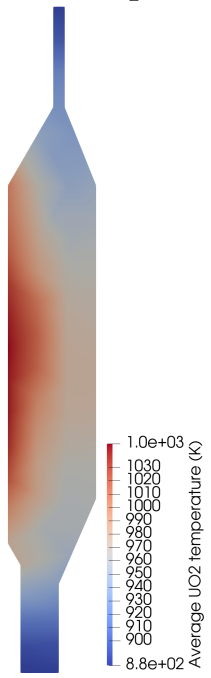

(h) Average $\mathrm{UO}_{2}$ temperature

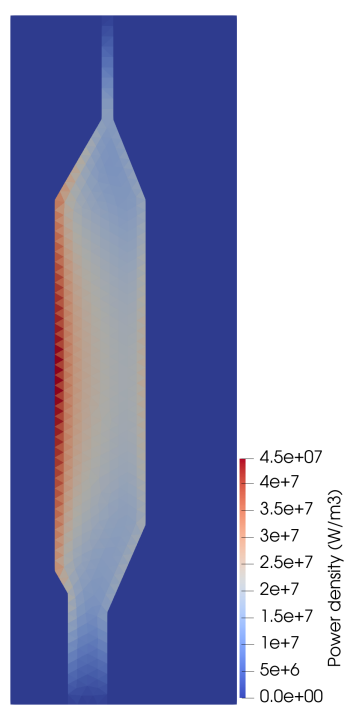

(c) Power density

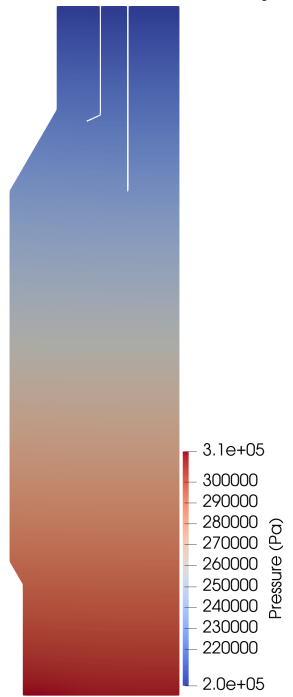

(f) Pressure distribution

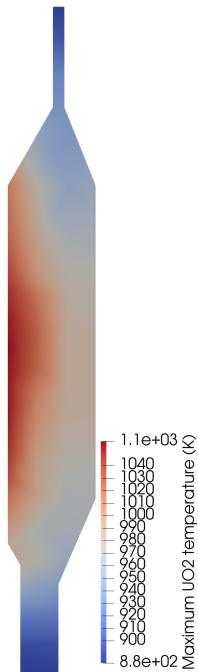

(i) Maximum $\mathrm{UO}_{2}$ temperature

Figure 27: Pronghorn-Griffin steady-state coupled simulation results for the Mk1-FHR 


\subsubsection{Transient Simulations}

The simulation of relevant accident scenarios is currently work in progress. The primary loop model under development at Argonne National Laboratory will enable accurate incoming flow conditions during the transient simulations.

\subsection{Future Work}

This model will be continuously improved and updated with the latest technology implemented in the modeling software at INL. The finite-element based thermal hydraulics model will be replaced by an equivalent finite-volume based model, for the reasons listed in Section 4.3. This will serve also serve as an additional code verification, and further study will highlight the comparative advantages of both methods. The updated multiphysics coupled simulation will also include a primary loop model currently in development. Using the work presented in Section 3, a realistic burnup distribution in the pebbles can be generated, which will then be used to generate equilibrium core group cross sections in Serpent 2. This will enable the comparison of accidental transient performance with both fresh and burned fuel, complimenting the work shown in [33]. 


\section{CONCLUSIONS}

This report details the progress and activities of INL for the NRC project "Development and Modeling Support for Advanced Non-Light Water Reactors," for which we report the successful completion of one task. In addition, the extended-scope tasks have also been completed. The following task completions are reported:

- Task 1d: A net radiation transfer method for modeling RCCS geometries is implemented into MOOSE; this capability is available to Pronghorn. For validation, preliminary models of NSTF experiment R022 and the HTTR VCS mockup are created and compared to measured data. These models use the net radiation transfer method to model radiative transfer, while natural convection is taken into account via correlations. The MOOSE NSTF results agree almost perfectly with STAR-CCM+ for the simplified model created for cross-validation, and reasonably well with temperatures measured along the heated cavity wall. The differences between predicted and measured temperatures are conjectured to result from the simplistic treatment of natural convection heat transfer, differences in modeled and as-built heat flux distributions on the heated wall, uncertainty resulting from digitization of the thermocouple temperatures, or a combination thereof. The RPV temperature shape in the VCS model agrees well with the experiment, except for underpredicting the top dome temperature. This difference stems from a heater surface temperature discrepancy that requires further investigation.

- Task 4c: An algorithm for computing the pebble-bed reactor equilibrium core isotopic composition is developed. The algorithm solves equations for (1) pebble volume fractions of pebbles with different burnups and different initial compositions and (2) the isotopic composition of each of these pebble groups, over 1-D streamlines. A prototype of the algorithm is implemented into the reactor multiphysics code Griffin. Initial results for a simplified axisymmetric pebble-bed reactor are presented. It is noted that the presented results are preliminary, since implementation of the equilibrium core depletion algorithm has not been thoroughly verified. Future work will focus on integrating the equilibrium core depletion algorithm tightly with Griffin, improving on the data transfer between multi-dimensional neutronics solves and 1-D depletion solves, as well as thorough verification.

- Task 7: A coupled neutronics-thermal hydraulics-1-D fuel performance model is constructed in the MOOSE framework using Griffin, Pronghorn, and the heat conduction module. This model can solve the steady-state multiphysics problem using reasonable computing resources. Initial comparisons of the diffusion neutronics model were performed against Monte Carlo simulations. The fuel performance model was previously validated using high-fidelity heterogeneous pebble models. The thermal-hydraulics model will be verified in future works via comparison to high-fidelity CFD simulations. The model is currently being expanded to support transient simulations, including reactivity insertion accidents and loss-of-flow transients. A primary loop model for the latter is being developed at Argonne National Laboratory. 


\section{References}

[1] H. Niessen and S. Ball, "Heat transport and afterheat removal for gas cooled reactors under accident conditions," Tech. Rep. IAEA-TECDOC-1163, IAEA, 2000.

[2] G. Atomics, "Gas turbine-modular helium reactor (GT-MHR) conceptual design description report," Tech. Rep. GA-910720, General Atomics, 1996.

[3] S. Saito, T. Tanaka, and Y. Sudo, "Design of high temperature engineering test reactor (httr)," tech. rep., Japan Atomic Energy Research Inst., 1994.

[4] Z. Wu, D. Lin, and D. Zhong, "The design features of the htr-10," Nuclear Engineering and Design, vol. 218, pp. 25-32, 2002.

[5] P. Williams, F. Silady, T. Dunn, R. Noren, D. McEachern, D. Dilling, J. Berkoe, F. Homan, L. Mears, S. Penfield Jr, et al., "MHTGR development in the United States," Progress in Nuclear Energy, vol. 28, no. 3, pp. 265-346, 1994.

[6] "RELAP5-3D Code Manual Volume I: Code Structure, System Models and Solution Methods," Tech. Rep. INL/MIS-15-36723, Revision 4.4, Idaho National Laboratory, 2018.

[7] D. Andrs et al., "RELAP-7 Level 2 Milestone Report: Demonstration of a Steady State Single Phase PWR Simulation with RELAP-7," Tech. Rep. INL/EXT-12-25924, Idaho National Laboratory, 2012.

[8] R. Hu, "SAM theory manual," Tech. Rep. ANL/NE-17/4, Argonne National Laboratory, 2017.

[9] A. Novak, R. Carlsen, S. Schunert, P. Balestra, R. Slaybaugh, and R. Martineau, "Pronghorn: A multidimensional coarse mesh application for advanced reactor thermal-hydraulics," submitted to Nucl. Techn.

[10] T. Drzewiecki et al., "The US NRC Advanced Gas REactor Evaluator (AGREE)," in The 14th International Topical Meeting on Nuclear Reactor Thermalhydraulics (NURETH-14), 2011.

[11] D. D. Lisowski, C. D. Gerardi, D. J. Kilsdonk, N. C. Bremer, S. W. Lomperski, R. Hu, and M. T. Farmer, "Final project report on rccs testing with air-based nstf," Tech. Rep. ANL-ART-47, Argonne National Lab. (ANL), 2016.

[12] Heat Transport and Afterheat Removal for Gas Cooled Reactors under Accident Conditions. No. 1163 in TECDOC Series, Vienna: International Atomic Energy Agency, 2001.

[13] D. R. Gaston, C. J. Permann, J. W. Peterson, A. E. Slaughter, D. Andrs, Y. Wang, M. P. Short, D. M. Perez, M. R. Tonks, J. Ortensi, L. Zou, and R. C. Martineau, "Physics-based multiscale coupling for full core nuclear reactor simulation," Annals of Nuclear Energy, vol. 84, pp. 45-54, 2015.

[14] M. Modest, Radiative Heat Transfer. Elsevier Science, 2013.

[15] L. Harbour et al., "Unstructured-mesh particle tracking and ray-tracing for multiphysics simulation in moose," in preparation, 2020.

[16] W. F. Walters, "Use of the chebyshev-legendre quadrature set in discrete-ordinate codes," in Conference: Computational methods in high temperature physics, 1987. 
[17] R. Hu, A. Kraus, M. Bucknor, Q. Lv, and D. Lisowski, "Final project report on computational modeling and analysis of air-based nstf,"

[18] M. Bucknor, R. Hu, D. Lisowski, and A. Kraus, "Comparisons of RELAP5-3D Analyses to Experimental Data from the Natural Convenction Shutdown Heat Removal Test Facility," in 2016 International Congress on Advances in Nuclear Power Plants (ICAPP 2016), 2016.

[19] M. Bahrami, "Natural convection." https://www.sfu.ca/ mbahrami/ENSC\%20388/Notes/ Natural\%20Convection.pdf. Online; accessed 19 November 2020.

[20] J. R. Lloyd and W. R. Moran, "Natural Convection Adjacent to Horizontal Surface of Various Planforms," Journal of Heat Transfer, vol. 96, pp. 443-447, 111974.

[21] S. Schunert, J. Ortensi, Y. Wang, Z. Prince, M. DeHart, V. Labouré, A. Lindsay, F. Kong, and R. Martineau, "NRC multiphysics analysis capability deployment fy2020 - part 1," INL report INL/EXT-19-56762, rev. 0, Idaho National Laboratory, 122019.

[22] L. Zou and R. A. Berry and R.C. Martineau and D. Andrs and H. Zhang and J.E. Hansel and J.P. Sharpe and R.C. Johns, “RELAP-7 Closure Correlations,” Tech. Rep. INL/EXT-17-41653, Idaho National Laboratory, 2017.

[23] M. Wolfstein, "The velocity and temperature distribution in one-dimensional flow with turbulence augmentation and pressure gradient," International Journal of Heat and Mass Transfer, vol. 12, no. 3, pp. 301-318, 1969.

[24] Shoji TAKADA and Yasuaki SHIINA and Yoshiyuki INAGAKI and Makoto HISHIDA and Yukio SUDO, "Benchmark problem for IAEA coordinated research program (CRP-3) on GCR afterheat removal (i)," Tech. Rep. JAERI-Research-95-056, 1995.

[25] R. Morris, “Cubit 15.0 user documentation," tech. rep., ETI, UT, 2014.

[26] D. B. Go, "Free convection overview," January 2014. Online, https://www3.nd.edu/ sst/ teaching/AME60634/lectures/AME60634_F13_lecture25.pdf, AME60634 class presentation University of Notre Dame.

[27] Y. Wang, S. Schunert, J. Ortensi, V. Laboure, M. DeHart, Z. Prince, F. Kong, J. Harter, P. Balestra, and F. Gleicher, "Rattlesnake: A moose-based multiphysics multi-scheme radiation transport application," submitted to Nucl. Techn.

[28] A. Kadak and M. Bazant, "Pebble flow experiments for pebble bed reactors," in 2nd International Topical Meeting on HIGH TEMPERATURE REACTOR TECHNOLOGY, 2004.

[29] F. Vitullo, J. Krepel, J. Kalilainen, H.-M. Prasser, and A. Pautz, “Statistical Burnup Distribution of Moving Pebbles in the High-Temperature Reactor HTR-PM," Journal of Nuclear Engineering and Radiation Science, vol. 6, 02 2020. 021108.

[30] C. H. Rycroft, A. Dehbi, T. Lind, and S. Güntay, "Granular flow in pebble-bed nuclear reactors: Scaling, dust generation, and stress," Nuclear Engineering and Design, vol. 265, pp. 69 84, 2013.

[31] G. Marleau, A. Hebert, and R. Roy, "A user guide for dragon version5," Tech. Rep. IGE-335, Ecole Polytechnique de Montreal, 102020. 
[32] A. et al., "Technical Description of the "Mark 1" Pebble-Bed Fluoride-Salt-Cooled HighTemperature Reactor (PB-FHR) Power Plant UCBTH-14-002," tech. rep., University of California Berkeley, 2014.

[33] X. Wang, Coupled neutronics and thermal-hydraulics modeling for pebble-bed Fluoride-Salt-Cooled, High-Temperature Reactor. PhD thesis, University of California Berkeley, 2018.

[34] A. Novak, Multiscale Thermal-Hydraulic Methods for Pebble Bed Reactors. PhD thesis, University of California Berkeley, 2020.

[35] J. Stempien, Tritium transport, corrosion, and Fuel performance modeling in the Fluoride Salt-Cooled High-Temperature Reactor. PhD thesis, Massachusetts Institute of Technology, 2015.

[36] K. Dolan, Tritium Retention in Nuclear Graphite, System-Level Transport, and Management Strategies for the Fluoride-Salt-Cooled High-Temperature Reactor. PhD thesis, Massachusetts Institute of Technology, 2020.

[37] C. W. Forsberg, "Market basis for salt-cooled reactors: Dispatchable heat, hydrogen, and electricity with assured peak power capacity," Nuclear Technology, vol. 0, no. 0, pp. 1-27, 2020.

[38] C. Forsberg, "Production of hydrogen using nuclear energy," in Safety and Economy of Hydrogen Transport: Second International Symposium, 2003.

[39] F. Rahnema, D. Diamond, D. Serghiuta, and P. Burke, "Phenomena, gaps and issues for neutronics modeling and simulation of FHRs," Annals of Nuclear Energy, vol. 123, pp. 172-179, 2019.

[40] M. Nakagawa and H. Inoue, "Double heterogeneity effect of fuel pin and subassembly in a fast power reactor," Nuclear Science and Engineering, vol. 83, pp. 214-228, 1983.

[41] X. Wang, K. Huff, M. Aufiero, P. Peterson, and M. Fratoni, "Coupled reactor kinetics and heat transfer model for fluoride salt-cooled high-temperature reactor transient analysis," in International Conference on Nuclear Engineering, pp. 26-06, 2016.

[42] A. Hebert, "A collision probability analysis of the double-heterogeneity problem," Nuclear Science and Engineering, vol. 115, 1993.

[43] R. Sanchez and G. Pomraning, "A statistical analysis of the double heterogeneity problem," Ann. Nucl. Energy, vol. 18, p. 371, 1991.

[44] D. J. Siefman, G. Girardin, A. Rais, A. Pautz, and M. Hursin, "Full Core modeling techniques for research reactors with irregular geometries using Serpent and PARCS applied to the CROCUS reactor," Annals of Nuclear Energy, vol. 85, pp. 434-443, 2015.

[45] K. Hadad, M. Nematolahi, and A. Golestani, "VVER-1000 cross-section library generation for ORIGEN-II based on MCNP calculations," International Journal of Hydrogen Energy, vol. 40, pp. 15158-15163, 2015.

[46] Q. Pan and K. Wang, "One-step monte carlo global homogenization based on rmc code," Nuclear Engineering and Technology, vol. 51, pp. 1209-1217, 2019.

[47] J. Leppanen, R. Mattila, and M. Pusa, "Validation of the Serpent-ARES code sequence using the MIT BEAVRS benchmark - Initial core at HZP conditions," Annals of Nuclear Energy, vol. 69, pp. 212-225, 2014. 
[48] K. Zhuang, X. Tang, and L. Cao, "Development and verification of a model for generation of MSFR few-group homogenized cross-sections based on a Monte Carlo code OpenMC," Annals of Nuclear Energy, vol. 124, pp. 187-197, 2019.

[49] E. Nikitin, E. Fridman, and K. Mikityuk, "Solution of the OECD/NEA neutronic SFR benchmark with Serpent-DYN3D and Serpent-PARCS code systems," Annals of Nuclear Energy, vol. 75, pp. 492-497, 2015.

[50] Z. Peng, Research on Physical Analysis Method and Physical Characteristics of Supercritical Water Reactor. PhD thesis, Tsinghua University, 2012.

[51] Y. Yuan, J. Xing, X. Huo, and K. Wang, "Research on the on-the-fly homogenization method based on RMC code for criticality calculations.," Annals of Nuclear Energy, vol. 135, 2020.

[52] J. Leppanen, “Serpent - a continuous-energy monte carlo reactor physics burnup calculation code," VTT Technical Research Centre of Finland, 2013.

[53] E. R. J. Woodcock and T. C. Longworth, "Techniques used in the gem code for monte carlo neutronics calculations in reactors and other systems of complex geometry," ANL-7050 Technical report, 1965.

[54] E. Fridman and J. Leppanen, "On the use of the serpent monte carlo code for few-group cross section generation," Annals of Nuclear Energy, vol. 38, pp. 1399-1405, 2011.

[55] Z. Li, L. Cao, H. Wu, Q. He, and W. Shen, "On the improvements in neutronics analysis of the unit cell for the pebble-bed fluoride-salt-cooled high-temperature reactor," Progress in Nuclear Energy, vol. 93, pp. 287-296, 2016.

[56] A. Novak, P. Romano, B. Wendt, R. Rahaman, E. Merzari, L. Kerby, C. Permann, R. Martineau, and R. Slaybaugh, "Preliminary Coupling of OpenMC and Nek5000 Within the MOOSE Framework," PHYSOR, 2018.

[57] P. B. et al., "PBMR-400 benchmark solution of exercise 1 and 2 using the MOOSE based applications: MAMMOTH, Pronghorn," PHYSOR 2020, Transition to a scalable nuclear future, 2020. 